Ideais em anéis de matrizes finitos e aplicações à Teoria de Códigos

Edite Taufer

TESE APRESENTADA

$\mathrm{AO}$

Instituto De MatemáticA E Estatística

DA

Universidade DE SÃo PAUlo

PARA

OBTENÇÃO DO TÍTULO

$\mathrm{DE}$

DOUTORA EM CiÊnCIAS

Programa: Matemática

Orientador: Prof. Dr. Francisco César Polcino Milies

São Paulo, Janeiro de 2018 


\section{Ideais em anéis de matrizes finitos e aplicações à Teoria de Códigos}

Esta versão da tese contém as correções e alterações sugeridas pela Comissão Julgadora durante a defesa da versão original do trabalho, realizada em 19/01/2018. Uma cópia da versão original está disponível no

Instituto de Matemática e Estatística da Universidade de São Paulo.

Comissão Julgadora:

- Prof. Dr. Francisco César Polcino Milies - IME-USP

- Prof. Dr. Raul Antonio Ferraz - IME-USP

- Prof. Dra. Mariana Cornelissen Hoyos - UFSJ

- Prof. Dr. Antonio Paques - UFRGS

- Prof. Dr. Rodrigo Lucas Rodrigues - UFC 


\section{Agradecimentos}

A mesma história contada por cada indivíduo é diferente...

Tudo começou em 2013 quando convidei o professor Antonio Paques, cujo codinome é "Paquito", para ir à FURG e avaliar um trabalho de Carla Rejane Fick Pinz e meu, chamado Dígitos Verificadores e Detecção de Erros. Aproveitei para pedir que me orientasse no doutorado e continuássemos com nosso trabalho desenvolvido no mestrado, intitulado O Problema Inverso de Galois: casos Cúbicos e Quínticos. Ele respondeu que poderíamos trabalhar, mas em "ação de grupóides e ações parciais de grupos...". A minha cara dizia o que meu coração sentia: não... Paquito perguntou: o que eu achava de continuar a trabalhar na Teoria de Códigos e com o professor C. Polcino Milies? Gostei!!! Pensei que era um grande desafio, e ia correr atrás... Em novembro de 2013 teria a V Jornada de Álgebra na FURG e Paquito convidou o professor Polcino para participar do encontro, ele aceitou... Depois de muito trabalho e aprendizado, cá estou após mais de quatro anos, para agradecer imensamente o carinho e a confiança que ambos depositaram em mim... Obrigada!

A primeira disciplina que assisti na USP foi Tópicos de Álgebra cujo professor foi o Raul Ferraz, e eu mal sabia que ele seria o meu melhor amigo, confidente e grande parceiro de trabalho. Obrigada!

Ao amigo Rogério Villafranca que fez e faz diferença na sua bondade em pronto atender com sua humildade que tanto ensina... principalmente em programar no SageMath, para que eu pudesse computar o peso dos códigos estudados nesta Tese, e ao amigo José A. Silva Neto que sempre esteve presente, principalmente ao me ajudar com o LaTeX. Obrigada!

Nosso grupo de trabalho está formado por: C. P. Milies, R. Ferraz, S. Assuena, A. Duarte, E. Iwaki, R. R. M. Silva, A. G. Chalom, R. Budaibes (Bud), J. A. Silva Neto (Sodé) e R. Villafranca. Além de parceiros, compartilhei boa parte de meu tempo, sendo na USP ou na UFABC. Muito obrigada pela amizade, pelos conselhos, ajuda e dedicação para comigo!

Aos meus familiares que sempre me apoiaram e entenderam a minha frequente falta: Telvi, Loide, Evaldo, Evandro, Magali, Nathali, Frederico e Hallan, Mariana, Digão e Aninha que vieram morar em São Paulo.

Aos meus professores do IME-USP, em especial: Gláucio Terra, Henrique Guzzo Junior e Alexandre Lymberopoulos. Aos amigos e colegas da FURG que além de aprovarem a minha saída para qualificação, sempre estiveram presentes, em especial: Denise Varella Martinez, Ricardo Giacobbo Luz, Luverci Do Nascimento Ferreira, os De Cezaro: Fabiana e Adriano, Lineia Schutz, Juliana Sar- 
tori Ziebell, Jorge Pimentel, Águeda Turatti. Aos amigos de longa data que estreitei laços: Gisele Boschetti, Patrícia L. Cunha e Rodrigo Bissacot e ao todynho Bibicio que é o pacotinho andante mais fofo e cheiroso dos últimos dois anos! Aos amigos que fiz: Auriluce Oliveira, Jorge de Lima, Majô da Silva, Felipe Nogueira, Sandra Grassmann Polcino, Ana Paula Sanches Veloso, Regiane Fascina Prado Jacintho Guimarães e outros tantos que têm a minha eterna gratidão!

Aos colegas e amigos que fiz no decorrer destes anos, são muitos e alguns com mais intensidade estiveram presentes em minha vida: Carlos Alexandre Gomes da Silva, Alexsandro Schneider, Felipe Felix, Giliard Souza, Thiago Grando, Larissa Sartori, Max Reinhold Jahnke, Adèle Ribeiro, Bruno Jacóia, Yuri Maluf, Paula Lavieri, Elivaldo Macedo, Cesar Batista, Ícaro Gonçalves, Adriana Vietmeier Nicoli, Jackeline Conrado, Carolina Lemos, Oscar Armando Hernández Morales e os colombianos, peruanos, iranianos, chilenos...

No espírito de Bobbie Carlyle e o homem que se busca de si mesmo, ela vê o positivo em cada situação e aprende com isso. Talvez o seu trabalho mais conhecido seja a peça de bronze intitulada Self Made Man, o homem que se constrói a si mesmo, a partir da pedra em bruto da qual ele emerge. Segundo Alexis Carrel, "O homem não pode fazer-se sem sofrer, pois é ao mesmo tempo o mármore e o escultor". "Ninguém sabe o que passei pra chegar até aqui..." mas sei que o hoje é realidade. O Amor é o alimento para uma vida feliz, com a confiança e o amparo de quem gosta da gente, a gente voa... Obrigada! 


\section{Resumo}

TAUFER, E. Ideais em anéis de matrizes finitos e aplicações à Teoria de Códigos. 2018. 53 ff. Tese (Doutorado) - Instituto de Matemática e Estatística, Universidade de São Paulo, São Paulo, 2018.

Neste trabalho damos uma descrição completa dos ideais à esquerda em anéis de matrizes sobre corpos finitos. Aplicamos estes resultados ao estudo de álgebras de grupo de uma família particular de grupos indecomponíveis e mostramos como construir códigos corretores de erros como ideais destas álgebras. Em particular, exibimos exemplos de códigos tais que, para um dado comprimento e uma dada dimensão, têm o melhor peso possível.

Palavras-chave: anel de matrizes, corpo finito, álgebra de grupo, códigos corretores de erros, código de grupo, melhor código. 


\section{Abstract}

TAUFER, E. Ideals in finite matrix rings and applications to Coding Theory. 2018. $53 \mathrm{ff}$. Tese (Doutorado) - Instituto de Matemática e Estatística, Universidade de São Paulo, São Paulo, 2018.

In this work we give a complete description of the left ideals in the full ring of matrices over a finite field. We apply these results to the study of group algebras of a given family of indecomposable groups and show how to construct error correcting codes as ideals of these algebras. In particular, we exhibit examples of codes such that, for a given length and a given dimension, have the best possible weight.

Keywords: matrix ring, finite field, group algebra, error correcting codes, group code, best code. 


\section{Introdução}

No fim da década de 40 do século XX, teve início com Richard W. Hamming e Claude Shannon, o estudo de códigos corretores de erros. Um código é um conjunto $\mathscr{C}$ de palavras que são sequências $\left(a_{0}, a_{1}, a_{2}, \ldots, a_{n-1}\right)$ cujo comprimento é $n$ e os seus elementos são tomados em um conjunto $A$ com cardinalidade $q$, denominado alfabeto.

Nessa época Hamming trabalhava no Bell Telephone Laboratories. Ali os computadores eram muito lentos e capazes apenas de detectar erros, não de corrigi-los. Quando isto acontecia, o computador parava de processar o programa que executava e passava para outro e então muitos trabalhos eram perdidos.

Em 1950 foi publicado no The Bell System Technical Journal [11] o estudo de Hamming, onde ele descreve um código capaz de detectar até dois erros e corrigir um, se ele for o único em cada palavra. Querendo construir um código ainda mais eficiente Hamming perguntou se seria possível construir um código capaz de detectar e corrigir um erro em uma palavra com quatro dígitos de informação e três de redundância. A resposta já havia sido dada no artigo A Mathematical Theory of Communication [20], de um colega de trabalho, chamado Claude Shannon em 1948.

O trabalho de Hamming e Shannon é considerado hoje como o responsável pela origem de duas teorias: a Teoria de Códigos Corretores de Erros e a Teoria da Informação.

Pouco tempo depois Marcel Golay, que trabalhava no Signal Corps Engineering Laboratories em Fort Monmouth, em Nova Jersey, inspirado no trabalho de Shannon publicou o artigo Notes on Digital Coding [7], de uma única página, onde descreve alguns códigos que estão entre os melhores utilizados até hoje.

Desde então, a Teoria de Códigos Corretores de Erros é um amplo campo de pesquisa que vem despertando o interesse de matemáticos, engenheiros elétricos e cientistas da computação.

A fim de se obter uma teoria matemática dos códigos é necessário introduzir alguma estrutura algébrica. Para isto, consideraremos o alfabeto sendo um corpo finito $\mathbb{F}$ com $q$ elementos e o código $\mathscr{C}$ será um subespaço vetorial do espaço $\mathbb{F}^{n}$. Desta forma, $\mathscr{C}$ é dito código linear $q$-ário de comprimento $n$.

Uma importante classe de códigos lineares são os códigos cíclicos, que possuem a seguinte propriedade: se $\left(c_{0}, c_{1}, c_{2}, \ldots, c_{n-1}\right)$ está em $\mathscr{C}$ então $\left(c_{n-1}, c_{0}, c_{1}, \ldots, c_{n-2}\right)$ também está no código. Os exemplos mais importantes dessa classe são os códigos de Hamming e de Golay.

O espaço vetorial $\mathbb{F}^{n}$ é isomorfo (como espaço vetorial) à álgebra de grupo $\mathbb{F} C_{n}$, onde $C_{n}$ é o grupo cíclico de ordem $n$. Em particular, um subespaço de $\mathbb{F} C_{n}$ corresponde a um código cíclico se, e somente se, ele for um ideal de $\mathbb{F} C_{n}$. 
De modo geral, um código de grupo é um ideal de uma álgebra de grupo finita $\mathbb{F} G$, onde $G$ é um grupo finito.

No caso em que a característica de $\mathbb{F}$ não divide a ordem de $G$, a álgebra de grupo correspondente é semissimples e, portanto, o estudo dos ideais desta álgebra é equivalente ao estudo dos elementos idempotentes. Este será o caso estudado neste trabalho.

Quando se constrói um código, há dois elementos importantes a serem considerados: Por um lado, deseja-se que o código seja suficientemente grande para poder transmitir muita informação (no caso dos códigos lineares, o subespaço que o define seja de dimensão a maior possível); Por outro lado, é importante que a distância mínima entre as palavras do código, seja a maior possível, para que o código tenha uma boa capacidade de correção de erros. Como o espaço total é finito, estes interesses são conflitantes. Esta situação é conhecida como o problema principal da Teoria de Códigos.

Existe uma tabela [10, Linear Codes] na qual, para cada corpo finito $\mathbb{F}$, para cada comprimento $n$ das palavras do código linear associado a cada dimensão possível, dá a melhor distância mínima já obtida (isto é, para a qual existe um código nestas condições) e uma cota superior para cada distância mínima em questão.

Na sua tese de doutorado S. Assuena [1] e a posteriori num artigo com C. Polcino Milies [2], foi mostrado que é possível igualar alguns dos recordes conhecidos, construindo códigos relativamente simples em Álgebras de Grupos Diedrais.

Em 1983, E. G. Goodaire [8], ao classificar Álgebras de Loop que são alternativas, introduziu o conceito de $S L C$-Grupos. Estes são grupos $G$, com centro $\mathscr{Z}(G)$, tais que $G / \mathscr{Z}(G) \cong C_{2} \times C_{2}$ ( o produto direto de dois grupos cíclicos de ordem 2) e tem um único comutador não trivial. Estes grupos têm desempenhado um papel fundamental no estudo de involuções de álgebras de grupo, e no caso finito foram completamente classificados por G. Leal e C. Polcino Milies [15] e por E. Jespers, G. Leal e C. Polcino Milies [14]. Na minha qualificação do doutoramento, descrevi os SLC-Códigos bem como os idempotentes geradores das componentes simples das álgebras de grupo associada a ele (os grupos indecomponíveis são de cinco famílias distintas).

No Capítulo 1 deste trabalho chamado Resultados Básicos, descrevemos como determinar o número de componentes simples e a dimensão de cada componente simples de uma certa álgebra de grupo semissimples. Terminamos explicitando a forma de encontrar os idempotentes centrais primitivos para grupos abelianos.

No trabalho de M. G. Cornelissen e C. Polcino Milies [3], são caracterizados os grupos indecomponíveis $\mathrm{G}$, tais que $G / \mathscr{Z}(G) \cong C_{p} \times C_{p}$ (produto direto de dois grupos cíclicos de ordem prima). Estes grupos, podem ser classificados no caso finito em cinco famílias cujos elementos não são isomorfos dois a dois, e são explicitados no Capítulo 2 deste trabalho, onde estudamos as álgebras de grupo de cada uma destas famílias. Mostramos que os códigos gerados por idempotentes centrais são combinatoriamente equivalentes a códigos abelianos, o que motiva o interesse em estudar ideais laterais. Além disso, se e é um idempotente central primitivo da parte não comutativa da álgebra $\mathbb{F} G$, provamos que $\mathbb{F} G e \cong M_{p}\left(\mathbb{F}_{i}\right)$, com $\mathbb{F}_{i}$ uma extensão de $\mathbb{F}$.

No Capítulo 3, damos atenção ao anel da matrizes $M_{n}\left(\mathbb{F}_{q}\right)$, de ordem $n$ sobre um corpo 
finito, que é semissimples. Nós computamos o número de ideais à esquerda deste anel, o número de idempotentes diferentes que geram o mesmo ideal à esquerda, e damos explicitamente um gerador idempotente para cada ideal à esquerda de um dado posto $k$, com $1 \leq k \leq n$.

Seja $e$ um idempotente central primitivo da parte não comutativa da álgebra $\mathbb{F} G$, onde $G$ é tal que $G / \mathscr{Z}(G) \cong C_{p} \times C_{p}$. No Capítulo 4, explicitamos o isomorfismo $\mathbb{F} G e \cong M_{p}\left(\mathbb{F}_{i}\right)$, para duas das cinco famílias de grupos considerados, e indicamos como estender estes resultados às outras famílias.

Dado um corpo $\mathbb{F}$ com $q$ elementos, fixados um comprimento $(n)$ e um peso $(p)$ é denotado por $A_{q}(n, p)$ o maior número possível de palavras $(M)$ para o qual existe um $(n, M, p)$-código $q$-ário. Um código, para o qual $M=A_{q}(n, p)$, é chamado um Código Ótimo. No Capítulo 5, considerando casos particulares quando $G / \mathscr{Z}(G) \cong C_{3} \times C_{3}$, obtemos exemplos de Códigos Ótimos, com isto, igualando ao recorde da tabela [10, Linear Codes].

No trabalho é utilizado um programa descrito no Capítulo 6, elaborado pelo colega Rogério Villafranca, no software livre e de código aberto denominado SageMath (SAGE, acrônimo em inglês para Sistema Algébrico e Geométrico de Experimentações), que é um software que possui recursos que abrangem muitas áreas, incluindo álgebra, combinatória, análise numérica, teoria dos números e cálculo. Este software acopla a seus algoritmos o software livre e de código aberto denominado GAP (Groups, Algorithms, Programming - um sistema para álgebra discreta computacional), o que o torna muito útil para nosso trabalho. Este programa nos fornece a distribuição de pesos do código de grupo, desde que informemos o corpo finito de coeficientes, o idempotente gerador do código e uma base para o mesmo. 


\section{Sumário}

Introdução $\quad$ vii

1 Resultados Básicos $\quad 1$

1.1 Número de componentes simples de $\mathbb{F} G \ldots \ldots \ldots \ldots \ldots$

1.2 Representação matricial de um grupo $G \ldots \ldots \ldots \ldots$. . . . . . . . . 4

1.3 Descenso de Galois . . . . . . . . . . . . . . . . . . . . . . . . . . 5

1.4 Subgrupos Cocíclicos . . . . . . . . . . . . . . . . . . . 6

2 Códigos em $\mathbb{F} G \quad 9$

2.1 Estrutura dos Códigos Centrais . . . . . . . . . . . . . . . . . . . . . 12

2.2 Códigos Laterais em $\mathbb{F} G \ldots \ldots \ldots \ldots$

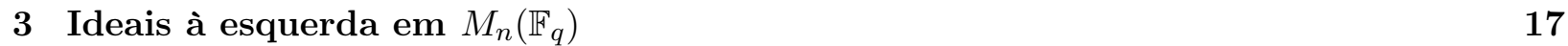

3.1 Ideais e subespaços . . . . . . . . . . . . . . . . 17

3.2 Idempotentes geradores de ideais à esquerda . . . . . . . . . . . . . . . . 20

4 Grupos dos tipos $\mathscr{D}_{1}$ e $\mathscr{D}_{2} \quad 25$

4.1 Correspondência na Álgebra das Matrizes para $\mathscr{D}_{1} \ldots \ldots \ldots \ldots \ldots \ldots$

4.2 Correspondência na Álgebra das Matrizes para $\mathscr{D}_{2} \quad \ldots \ldots \ldots$

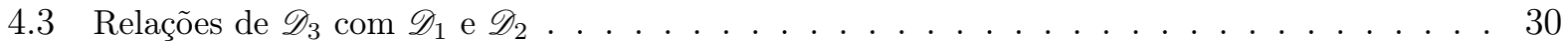

$\begin{array}{lll}5 & \text { Exemplos } & 37\end{array}$

6 Roteiro para o software SageMath $\quad 49$

6.1 Comandos para o SageMath . . . . . . . . . . . . . . . . . . . . . . . 49

6.2 Algoritmo SageMath . . . . . . . . . . . . . . . . . . . . 51

Referências Bibliográficas $\quad 53$ 


\section{Capítulo 1}

\section{Resultados Básicos}

Destacamos alguns resultados relevantes para este trabalho, os quais podem ser encontrados no livro An Introduction to Group Rings [17]. Todos os anéis considerados neste trabalho são anéis com unidade.

Definição 1.1. Dados $R$ um anel comutativo, $G$ um grupo e $\alpha=\sum_{g \in G} \alpha_{g} g$ um elemento da álgebra de grupo RG. Chama-se suporte de $\alpha$ ao conjunto

$$
\operatorname{supp}(\alpha)=\left\{g \in G ; \alpha_{g} \neq 0\right\}
$$

Definição 1.2. ([17], Def.3.3.1, p.134) Sejam $G$ um grupo e $R$ um anel, denotamos $S(G)$ o conjunto de todos os subgrupos de $G$. Para $H \in S(G)$, denotamos $\triangle_{R}(G, H)$ o ideal à esquerda de RG gerado pelo conjunto $\{h-1 ; h \in H\}$, isto é,

$$
\triangle_{R}(G, H)=\left\{\sum_{h \in H} \alpha_{h}(h-1) ; \alpha_{h} \in R G\right\} .
$$

O homomorfismo $\phi: R G \rightarrow R$ definido por $\phi\left(\sum_{g \in G} \alpha_{g} g\right)=\sum_{g \in G} \alpha_{g}$ é chamada de função aumento de $R G$ em $R$, e o conjunto

$$
\operatorname{Ker}(\phi)=\left\{\sum_{g \in G} \alpha_{g}(g-1) ; \alpha_{g} \in R, g \in G, g \neq 1\right\}=\triangle_{R}(G)
$$

é chamado de ideal de aumento de $R G$.

O próximo resultado é encontrado em [17, Lem.3.6.6, p.153], aqui mudaremos notação e denotamos por $|H|$ o número de elementos do conjunto $H$, e $H \triangleleft G$ significa que $H$ é subgrupo normal de um grupo $G$.

Lema 1.3. Sejam $R$ um anel e $H$ um subgrupo de um grupo $G$. Se $|H|$ é invertivel em $R$, então $e_{H}=\widehat{H}=\frac{1}{|H|} \sum_{h \in H} h$ é um idempotente de RG. Mais ainda, se $H \triangleleft G$ então $e_{H}$ é central.

O próximo resultado é frequentemente usado em nosso trabalho

Proposição 1.4. ([17], Prop.3.6.7, p.153) Sejam $R$ um anel e $H \triangleleft G$. Se $|H|$ é invertivel em $R$, com $e_{H}=\widehat{H}$ temos que

$$
R G=R G e_{H} \oplus R G\left(1-e_{H}\right)
$$


onde $R G e_{H} \cong R(G / H)$ e $\triangle_{R}(G, H)=R G\left(1-e_{H}\right)$.

E em particular:

Proposição 1.5. ([17], Prop.3.6.11, p.154) Seja RG uma álgebra de grupo semissimples. Se $G^{\prime}$ denota o subgrupo comutador de $G$, então podemos escrever

$$
R G=R G e_{G^{\prime}} \oplus \triangle_{R}\left(G, G^{\prime}\right)
$$

onde $R G e_{G^{\prime}} \cong R\left(G / G^{\prime}\right)$ é a soma de todas as componentes simples comutativas de $R G$ e $\triangle_{R}\left(G, G^{\prime}\right)=$ $R G\left(1-e_{G^{\prime}}\right)$ é a soma de todas as demais.

O teorema seguinte garante que as álgebras de grupo de nosso trabalho são semissimples

Teorema 1.6. (Teorema de Maschke,[17], Thm.3.4.7, p.140)

Seja $G$ um grupo. Então a álgebra de grupo $R G$ é semissimples se, e somente se,

(i) $R$ é semissimples;

(ii) $G$ é finito;

(iii) $|G|$ é invertivel em $R$.

O Teorema que segue é bem conhecido

Teorema 1.7. (Wedderburn-Artin, [17], Thm.2.6.18, p.104) Um anel $R$ é semissimples se, $e$ somente se, é uma soma direta de álgebra de matrizes sobre anéis de divisão

$$
R \cong M_{n_{1}}\left(D_{1}\right) \oplus M_{n_{2}}\left(D_{2}\right) \oplus \cdots \oplus M_{n_{s}}\left(D_{s}\right) .
$$

A tradução do Teorema de Wedderburn-Artin no contexto de álgebra de grupo é encontrado em [17, Thm.3.4.9, p.142], a seguir enunciado a parte de interesse neste trabalho.

Teorema 1.8. Sejam $G$ um grupo finito e $\mathbb{F}$ um corpo finito, tal que char $(\mathbb{F}) \nmid|G|$. Então:

(i) $\mathbb{F} G$ é a soma direta de um número finito de ideais $\left\{B_{i}\right\}_{1 \leqslant i \leqslant r}$ que como anéis são simples, e eles são as componentes simples de $\mathbb{F} G$.

(ii) Qualquer ideal bilateral de $\mathbb{F} G$ é uma soma direta de alguns membros da família $\left\{B_{i}\right\}_{1 \leqslant i \leqslant r}$;

(iii) Cada componente simples $B_{i}$ é isomorfa a um anel de matrizes $M_{n_{i}}\left(D_{i}\right)$ sobre um anel de divisão $D_{i}$, e $\mathbb{F} G \cong \oplus_{i=1}^{r} M_{n_{i}}\left(D_{i}\right)$ é um isomorfismo de $\mathbb{F}$-álgebras.

Seja $\mathbb{F} G$ uma álgebra semissimples escrita na forma

$$
\mathbb{F} G=B_{1} \oplus B_{2} \oplus \cdots \oplus B_{s} .
$$

onde $B_{i}=\mathbb{F} G e_{i}(1 \leq i \leq s)$ e $e_{1}, e_{2}, \ldots, e_{s}$ são idempotentes centrais primitivos. Se $e \in \mathbb{F} G$ é um idempotente central, então $I=\mathbb{F} G e$ é um ideal bilateral que sempre pode-se escrever como soma, de modo único, na forma $B_{i_{1}} \oplus B_{i_{2}} \oplus \cdots \oplus B_{i_{r}}$. Assim, $e=e_{i_{1}}+e_{i_{2}}+\cdots+e_{i_{r}}$ é soma única de idempotentes centrais primitivos, que são chamados seus constituintes.

\subsection{Número de componentes simples de $\mathbb{F} G$}

Denotamos por $\mathbb{F}$ um corpo finito com $q$ elementos, onde $q$ é uma potência de um primo e $G$ um grupo finito, tal que a álgebra de grupo $\mathbb{F} G$ é semissimples. A classe de conjugação de um elemento 
$a \in G$ será denotada por:

$$
\mathscr{C}_{a}:=\left\{g^{-1} a g, g \in G\right\} .
$$

Definição 1.9. ([17], Def.3.6.1, p.150) Sejam $G$ um grupo, $R$ um anel comutativo e $\left\{\mathscr{C}_{i}\right\}_{i \in I}$ o conjunto das classes de conjugação de $G$ que contêm somente um número finito de elementos. Para cada indice $i \in I$, denotemos por $\gamma_{i}=\sum_{x \in \mathscr{C}_{i}} x$, estes elementos são chamados de somas da classe de G sobre R.

Teorema 1.10. ([17], Thm.3.6.2, p.151) Sejam $G$ um grupo e $R$ um anel comutativo. Então, o conjunto $\left\{\gamma_{i}\right\}_{i \in I}$ de todas as somas de classes formam uma base de $\mathscr{Z}(R G)$, o centro de $R G$, sobre $R$.

Teorema 1.11. ([17], Thm.3.6.3, p.151) Sejam G um grupo finito e $K$ um corpo algebricamente fechado, tal que car $(\mathbb{K}) \nmid|G|$. Então, o número de componentes simples de $\mathbb{K} G$ é igual ao número de classes de conjugação de $G$.

Definição 1.12. ([17], Def.3.6.4, p.152) Um corpo $\mathbb{E}$ é chamado corpo de decomposição de um grupo $G$, se $\mathbb{E} G$ é isomorfa à soma direta de matrizes do anel das matrizes sobre $\mathbb{E}$.

Definição 1.13. Denominamos o expoente de um grupo $G$ ao menor inteiro positivo $n$, tal que $g^{n}=1$, para todo $g \in G$, e é denotado por $\exp (G)=n$.

Teorema 1.14. ([12], Cor.9.15, p.151)

Sejam $G$ um grupo de expoente $n$ e assumamos que o polinômio $x^{n}-1$ se decompõe em fatores lineares sobre o corpo $\mathbb{E}$. Se $\mathbb{E}$ tem característica prima, então é o corpo de decomposição para $G$.

Teorema 1.15. Sejam $G$ um grupo finito e $\mathbb{E}$ um corpo de decomposição de $G$, tal que char $(\mathbb{E}) \nmid|G|$. Então, o número de componentes simples de $\mathbb{E} G$ é igual ao número de classes de conjugação de $G$.

Para determinar o número de componentes simples e a dimensão de cada componente simples de uma álgebra de grupo semissimples, no caso em que $\mathbb{E}$ não é o corpo de decomposição para $G$, basta consultar em [4, Teorema 1.3]. O próximo teorema é uma adaptação ao caso semissimples. Antes de enunciá-lo, é necessário introduzir algumas definições.

Para todo elemento $g$ de um grupo $G$, denotaremos por $o(g)$ a ordem do elemento $g \in G$.

Definição 1.16. Chama-se a $\mathbb{F}$-classe ciclotômica correspondente a um elemento $g \in G$, ou uma $\mathbf{q}$-classe de $\boldsymbol{g}$, ao conjunto

$$
C_{\mathbb{F}}(g)=\left\{g, g^{q}, g^{q^{2}}, \cdots, g^{q^{t-1}}\right\},
$$

onde t é o menor inteiro positivo tal que $q^{t} \equiv 1(\bmod o(g))$.

Definição 1.17. Seja $a \in G$. Definimos a $\mathbb{F}$-classe ciclotômica de $\mathscr{C}_{a}$ como sendo

$$
S_{a}=\left\{\mathscr{C}_{a}, \mathscr{C}_{a^{q}}, \cdots, \mathscr{C}_{a^{q^{t^{\prime}-1}}}\right\}
$$

onde $t^{\prime}$ é o menor inteiro positivo tal que a é conjugado a $a^{q^{t^{\prime}}}$. 
Teorema 1.18. Sejam $\mathbb{F}$ um corpo com q elementos, $G$ um grupo finito e sejam $S_{1}, S_{2}, \cdots, S_{t}$ as $\mathbb{F}$-classes ciclotômicas das classes de conjugação de $G$. Então $\mathbb{F} G$ tem precisamente $t$ componentes simples e se $K_{1}, K_{2}, \ldots, K_{t}$ são as componentes simples de $\mathscr{Z}(\mathbb{F} G)$ então, com uma ordenação adequada dos indices, temos que $\left|S_{i}\right|=\left[K_{i}: \mathbb{F}\right]$, isto é, o indice de $\mathbb{F}$ em $K_{i}$ é o número de elementos de $S_{i}$.

Naturalmente nos perguntamos: quais são os idempotentes primitivos centrais geradores de cada componente? A fim de responder tal questão, descreveremos alguns resultados da teoria de Representações que serão suficientes para nosso trabalho e também explicitaremos alguns tópicos sobre Caracteres.

Apesar de que trabalharemos com certos p-grupos não comutativos, as técnicas que desenvolveremos permitem trabalhar apenas com os centros de tais grupos. Por isso, daremos ênfase a resultados sobre grupos abelianos.

\subsection{Representação matricial de um grupo $G$}

Nesta seção temos por referência [13].

Sejam $G$ um grupo e $\mathbb{F}$ um corpo. Denotamos por $G L(n, \mathbb{F})$, o grupo das matrizes invertíveis de ordem $n$ com entradas em $\mathbb{F}$. Uma representação de $G$ sobre $\mathbb{F}$ de grau $n$ é um homomorfismo $\rho: G \rightarrow G L(n, \mathbb{F})$. O grau da representação $\rho$ será denotado por $\operatorname{gr}(\rho)$.

Visto que cada representação é um homomorfismo, temos $\rho\left(1_{G}\right)=I_{n}$, onde $I_{n}$ denota a matriz identidade de ordem $n$, e $\rho\left(g^{-1}\right)=\rho(g)^{-1}$, para todo $g \in G$. O núcleo de uma representação $\rho$ é o conjunto $\operatorname{ker}(\rho)=\left\{g \in G ; \rho(g)=I_{n}\right\}$. Uma representação $\rho$ é dita fiel se $\operatorname{ker}(\rho)=\left\{1_{G}\right\}$, isto é, se o elemento identidade $1_{G}$ de $G$ é o único elemento de $G$ tal que $\rho\left(1_{g}\right)=I_{n}$.

Considerando uma representação $\rho: G \rightarrow G L(n, \mathbb{F})$ tal que $\operatorname{char}(\mathbb{F}) \nmid|G|$, a função $\chi: G \rightarrow \mathbb{F}$ definida por $\chi(g)=\operatorname{tr}(\rho(g))$, isto é o traço da matriz $\rho(g)$, é chamada de caracter da representação de $G$. O grau da representação $\rho$ também é chamado de grau do caracter $\chi$, e denotado por $\operatorname{gr}(\chi)$. No caso de $\operatorname{gr}(\chi)=1$, temos que $\chi=\rho$ e $\chi$ é chamado de caracter linear. Caracteres são constantes nas classes de conjugação de $G$, isto é, se $x$ e $y$ são elementos conjugados do grupo $G$, então $\chi(x)=\chi(y)$, para todo caracter $\chi \operatorname{de} G$.

Se $\mathbb{E}$ é corpo de decomposição para um grupo abeliano $G$, então

$$
\mathbb{E} G=\underbrace{\mathbb{E} \oplus \mathbb{E} \oplus \cdots \oplus \mathbb{E}}_{|G| \text { vezes }} .
$$

Logo, $G$ tem $|G|$ representações diferentes, que são as diferentes projeções de $\mathbb{E} G$ sobre cada componente.

Sejam $G=\left\langle a ; a^{m}=1\right\rangle$ um grupo cíclico de ordem $m$ e $\theta$ uma raiz $m$-ésima primitiva da unidade sobre $\mathbb{F}$. Então, $\mathbb{E}=\mathbb{F}(\theta)$ é um corpo de decomposição de $G$ e os $m$ caracteres de $G$ sobre $\mathbb{E}$ são:

$$
\begin{aligned}
& \rho_{j}: G \quad \rightarrow \quad \mathbb{E} \quad 0 \leqslant j \leqslant m-1 \\
& \rho_{j}\left(a^{k}\right) \mapsto \theta^{j k} \quad 0 \leqslant k \leqslant m-1 .
\end{aligned}
$$




\subsection{Descenso de Galois}

Com as notações acima, temos que a função

$$
\begin{aligned}
\sigma: \mathbb{E} & \rightarrow \mathbb{E} \\
\alpha & \mapsto \alpha^{q}
\end{aligned}
$$

é um automorfismo de $\mathbb{E}$, pois para quaisquer $x, y \in \mathbb{E}$ tem-se que:

$$
\begin{aligned}
(x+y)^{q} & =x^{q}+y^{q} \\
(x y)^{q} & =x^{q} y^{q} .
\end{aligned}
$$

Ainda, $\sigma(\alpha)=\alpha$ se, e somente se, $\alpha \in \mathbb{F}$.

Note que se $\sigma^{k}(\alpha)=\alpha^{q^{k}}$, para todos $\alpha \in \mathbb{E}$ e $k$ inteiro positivo, então $\sigma^{k}=I$ (identidade) se, e somente se, $\alpha^{q^{k}}=\alpha$, para todo $\alpha \in \mathbb{E}$; e isto acontece se $k=m$, pois $\mathbb{E}$ tem $q^{m}$ elementos, e este é o menor inteiro positivo nessas condições. Logo, $o(\sigma)=m$. Denotamos por $\operatorname{Gal}(\mathbb{E} / \mathbb{F})$ o grupo dos automorfismos Galois de $\mathbb{E}$ que fixam os elementos de $\mathbb{F}$. Como $|\operatorname{Gal}(\mathbb{E} / \mathbb{F})|=[\mathbb{E}: \mathbb{F}]=m$, temos que $\operatorname{Gal}(\mathbb{E} / \mathbb{F})=\langle\sigma\rangle$ é cíclico.

A função explicitada acima é chamada de automorfismo de Frobenius. Esta definição pode ser estendida para álgebras de grupo: Dados $G$ um grupo finito e $\alpha=\sum_{g \in G} \alpha_{g} g \in \mathbb{E} G$, definimos $\sigma(\alpha)=\sum_{g \in G} \sigma\left(\alpha_{g}\right) g \in \mathbb{E} G$; a função determinada por $\sigma$ é um automorfismo de $\mathbb{E} G$ que fixa os elementos de $\mathbb{F} G$.

O Descenso de Galois é um método para obter os idempotentes centrais de $\mathbb{F} G$ a partir dos idempotentes centrais de $\mathbb{E} G$. É o que mostraremos nos parágrafos seguintes:

Consideremos $\mathbb{E}=\mathbb{F}(\theta)$ o corpo de decomposição de $G$ com $G a l(\mathbb{E} / \mathbb{F})=\langle\sigma\rangle$. Temos $\mathbb{E} G=$ $M_{n_{1}}(\mathbb{E}) \oplus M_{n_{2}}(\mathbb{E}) \oplus \cdots \oplus M_{n_{s}}(\mathbb{E})$ e cada componente simples da álgebra é gerada por um idempotente central $e_{i} \in \mathbb{E} G$ primitivo, ou seja, $\mathbb{E} G e_{i} \cong M_{n_{i}}(\mathbb{E})$, com $1 \leq i \leq s$.

Como $\sigma: \mathbb{E} G \rightarrow \mathbb{E} G$ é um automorfismo, se $e_{i}$ é um idempotente central primitivo então $\sigma^{k}\left(e_{i}\right)$ também o é, para todo inteiro positivo $k$. Seja $l$ o menor inteiro positivo tal que $\sigma^{l}\left(e_{i}\right)=e_{i}$. Então $E_{i}=e_{i}+\sigma\left(e_{i}\right)+\sigma^{2}\left(e_{i}\right)+\cdots+\sigma^{l-1}\left(e_{i}\right)$ é um idempotente central; e se $E_{i} \neq E_{j}$, pode-se ver que $E_{i} E_{j}=0$. Como $\operatorname{Gal}(\mathbb{E} / \mathbb{F})=\langle\sigma\rangle, E_{i}$ é fixo pela extensão linear de $\operatorname{Gal}(\mathbb{E} / \mathbb{F})$ a $\mathbb{E} G$, donde $E_{i} \in \mathbb{F} G$.

Nos falta mostrar que $E_{i} \in \mathbb{F} G$ é primitivo. Suponhamos que não o seja, isto é, que $E_{i}=$ $e^{\prime}+e^{\prime \prime} \in \mathbb{F} G$. Mas, $e^{\prime}+e^{\prime \prime}=e_{i}+\sigma\left(e_{i}\right)+\sigma^{2}\left(e_{i}\right)+\cdots+\sigma^{l-1}\left(e_{i}\right)$ em $\mathbb{E} G$ é soma de idempotentes centrais primitivos. Pela unicidade da decomposição em idempotentes centrais primitivos, conforme visto ao final da primeira seção deste capítulo, $e^{\prime}$ e $e^{\prime \prime}$ devem ser somas parciais da soma acima:

$$
\begin{gathered}
e^{\prime}=\sigma^{k_{1}}\left(e_{i}\right)+\sigma^{k_{2}}\left(e_{i}\right)+\cdots+\sigma^{k_{t}}\left(e_{i}\right) \\
e^{\prime \prime}=\sigma^{k_{1}^{\prime}}\left(e_{i}\right)+\sigma^{k_{2}^{\prime}}\left(e_{i}\right)+\cdots+\sigma^{k_{l-t}^{\prime}}\left(e_{i}\right) .
\end{gathered}
$$

Mas para $\sigma\left(e^{\prime}\right)$, existiria algum $\sigma^{k_{j}}\left(e_{i}\right)$ que seria levado em algum $\sigma^{k_{r}^{\prime}}\left(e_{i}\right)$, donde $e^{\prime}$ não seria fixo pelas extensões a $\mathbb{E} G$ de elementos de $\operatorname{Gal}(\mathbb{E} / \mathbb{F})$, o que seria uma contradição. 


\subsection{Subgrupos Cocíclicos}

Neste trabalho as álgebras de grupo consideradas são sempre semissimples. Esta seção é dedicada a maneira de como determinar os idempotentes centrais primitivos que geram as componentes simples desta álgebra, no caso comutativo.

Definição 1.19. Seja $G$ um grupo finito.

Um subgrupo próprio $H$ de $G$ é chamado de subgrupo cocíclico se o quociente $G / H$ é cíclico.

Dados um grupo finito abeliano $A$ e o grupo multiplicativo do corpo dos números complexos $\mathbb{C}^{*}$, definimos $A^{*}=\left\{f: A \rightarrow \mathbb{C}^{*}\right.$; f é um homomorfismo de grupos $\}$. Naturalmente $A^{*}$ é grupo abeliano sob o produto de funções $f g(a)=f(a) g(a)$, para todo $a \in A$. Consideremos $A=\left\langle a_{1}\right\rangle \times$ $\left\langle a_{2}\right\rangle \times \cdots \times\left\langle a_{k}\right\rangle$, com $\left|\left\langle a_{i}\right\rangle\right|=n_{i}>0$, uma decomposição em produto direto.

O que segue, é inspirado em ([18], Cap.10, p.340), onde mostraremos que $A \cong A^{*}$. E faremos uso deste resultado, para provar que o número de subgrupos cíclicos de um grupo abeliano $A$ é o mesmo de subgrupos cocíclicos.

Vamos mostrar que $A \cong A^{*}$ :

Dado $f \in A^{*}$, observemos que basta calcular o valor de $f$ em cada gerador $a_{i} \in A$, e como $a_{i}^{n_{i}}=1$, em $\mathbb{C}^{*}$ podemos associar a $\theta_{n_{i}}$ uma raíz $n_{i}$-ésima primitiva da unidade.

Afirmação: $A^{*}=\left\langle f_{1}\right\rangle \times\left\langle f_{2}\right\rangle \times \ldots \times\left\langle f_{k}\right\rangle$, onde $f_{i} \in A^{*}, i \in\{1,2, \ldots, k\}$ é dada por

$$
f_{i}\left(a_{j}\right)=\left\{\begin{array}{l}
\theta_{n_{i}}, \text { se } i=j \\
1, \text { se } i \neq j
\end{array} .\right.
$$

Primeiramente mostraremos que cada $f \in A^{*}$ se escreve da seguinte forma $f=f_{1}^{l_{1}} f_{2}^{l_{2}} \ldots f_{k}^{l_{k}}$.

Temos que $A=\left\langle a_{1}\right\rangle \times\left\langle a_{2}\right\rangle \times \cdots \times\left\langle a_{k}\right\rangle$ e $f: A \rightarrow \mathbb{C}^{*}$. Então, dado $a=a_{1}^{r_{1}} a_{2}^{r_{2}} \ldots a_{k}^{r_{k}} \in A$ segue que $f(a)=f\left(a_{1}^{r_{1}}\right) f\left(a_{2}^{r_{2}}\right) \ldots f\left(a_{k}^{r_{k}}\right)$.

Note que $f\left(a_{i}^{r_{i}}\right)^{n_{i}}=f_{i}\left(a_{i}^{r_{i} n_{i}}\right)=1$, o que implica que $f\left(a_{i}\right)$ é uma raiz $n_{i}$-ésima primitiva da unidade, ou seja, $f\left(a_{i}\right) \in\left\langle\theta_{n_{i}}\right\rangle$. Assim, $f\left(a_{i}\right)=\theta_{n_{i}}^{l_{i}}=f_{i}^{l_{i}}\left(a_{i}\right)$. Portanto, $f\left(a_{i}^{r_{1}}\right)=f_{i}^{l_{i}}\left(a_{i}^{r_{1}}\right)$, para todo $1 \leqslant i \leqslant k$.

Donde, $f(a)=f_{1}^{l_{1}}\left(a_{1}^{r_{1}}\right) f_{2}^{l_{2}}\left(a_{2}^{r_{2}}\right) \ldots f_{k}^{l_{k}}\left(a_{k}^{r_{k}}\right)=f_{1}^{l_{1}}(a) f_{2}^{l_{2}}(a) \ldots f_{k}^{l_{k}}(a)$, que implica $f=f_{1}^{l_{1}} f_{2}^{l_{2}} \ldots f_{k}^{l_{k}}$.

No que segue, mostraremos que cada $f \in A^{*}$ se escreve de forma única.

Suponhamos que $f_{1}^{r_{1}} f_{2}^{r_{2}} \ldots f_{k}^{r_{k}}(a)=f_{1}^{t_{1}} f_{2}^{t_{2}} \ldots f_{k}^{t_{k}}(a)$, onde $1 \leqslant r_{i}, t_{i} \leqslant n_{i}$, para todo $a \in A$.

Observemos que basta calcular o valor de $f$ em cada gerador $a_{i} \in A$.

Então, $f_{1}^{r_{1}} f_{2}^{r_{2}} \ldots f_{k}^{r_{k}}\left(a_{i}\right)=f_{1}^{t_{1}} f_{2}^{t_{2}} \ldots f_{k}^{t_{k}}\left(a_{i}\right)$, o que implica $f_{i}^{r_{i}}\left(a_{i}\right)=f_{i}^{t_{i}}\left(a_{i}\right)$, para todo $1 \leqslant i \leqslant k$, daí $\theta_{n_{i}}^{r_{i}}=\theta_{n_{i}}^{t_{i}}$. Portanto, $r_{i}=t_{i}$, para todo $1 \leqslant i \leqslant k$.

Conforme o exposto acima, definindo o homomorfismo de grupos que leva gerador em gerador, prova-se que $A \cong A^{*}$.

Além disso, queremos mostrar que a cada subgrupo cocíclico corresponde um cíclico. De maneira geral: para $H$ subgrupo do grupo abeliano $A$ definimos o conjunto anulador de $H$, isto é, $H^{\circ}=$ $\left\{f \in A^{*} ; f(h)=1\right.$, para todo $\left.h \in H\right\}$. Mostraremos que $(A / H)^{*} \cong H^{\circ}$ : 
Seja $\omega: A \rightarrow A / H$ o epimorfismo canônico, e dada $\varphi: A / H \rightarrow \mathbb{C}^{*} \in(A / H)^{*}$. Definimos

$$
\begin{aligned}
\Psi:(A / H)^{*} & \rightarrow H^{\circ} \\
\varphi & \mapsto \Psi(\varphi)=\varphi \circ \omega .
\end{aligned}
$$

Primeiramente note que $\operatorname{Im}(\Psi) \subset H^{\circ}$. De fato,

$$
(\Psi(\varphi))(h)=(\varphi \circ \omega)(h)=1, \text { para todo } h \in H .
$$

- $\Psi$ é homomorfismo: sejam $\varphi_{1}, \varphi_{2} \in(A / H)^{*}$ então

$$
\Psi\left(\varphi_{1} \cdot \varphi_{2}\right)=\left(\varphi_{1} \cdot \varphi_{2}\right) \circ \omega=\varphi_{1}(\omega) \cdot \varphi_{2}(\omega) .
$$

- $\Psi$ é injetora: se $\varphi \in \operatorname{ker}(\Psi)$ então, $\Psi(\varphi)=1$ segue que

$$
\Psi(\varphi)(a)=1 \Leftrightarrow \varphi \circ \omega(a)=1 \Leftrightarrow \varphi(\bar{a})=1, \forall a \in A,
$$

donde $\varphi$ é a função constante.

- $\Psi$ é sobrejetora: seja $f \in H^{\circ}$ qualquer, e definimos

$$
\begin{aligned}
\varphi: A / H & \rightarrow \mathbb{C}^{*} \\
\bar{a} & \rightarrow \varphi(\bar{a})=f(a) .
\end{aligned}
$$

Vamos mostrar que $\varphi$ está bem definida:

Sejam $a_{1}, a_{2} \in A$ tais que $\overline{a_{1}}=\overline{a_{2}}$ assim, $a_{1}=a_{2} h$, para algum $h \in H$.

Logo, $f\left(a_{1}\right)=f\left(a_{2} h\right)=f\left(a_{2}\right) f(h)=f\left(a_{2}\right)$.

Além disso, $f(a)=\varphi(\bar{a})=(\varphi \circ \omega)(a)=(\Psi(\varphi))(a)$ o que implica $f(a)=\Psi(\varphi)(a)$, para todo $a \in A$.

Portanto, $\Psi$ é um isomorfismo.

Como $(A / H)^{*} \cong A / H$, segue que $A / H \cong H^{\circ}$. E se $H$ é cocíclico em $A$, então $H^{\circ}$ é cíclico em $A^{*}$.

Analogamente, temos que $A^{*} / H^{\circ} \cong H$, ou seja, todo subgrupo cocíclico $H^{\circ}$ em $A^{*}$ corresponde a um subgrupo cíclico $H$ em $A$.

Além dos tipos de idempotentes definidos pelo Lema 1.3, lembrando que $\mathbb{F}$ um corpo com $q$ elementos, temos o seguinte:

Lema 1.20 ([6], Lem.3). Sejam $G$ um grupo cíclico de ordem $p^{n}, n \geq 1$, e

$$
G=G_{0} \supset G_{1} \supset G_{2} \supset \cdots \supset G_{n}=1
$$

a cadeia descendente de todos os subgrupos de G. Então os elementos

$$
e_{0}=\widehat{G} \quad e \quad e_{i}=\widehat{G_{i}}-\widehat{G_{i-1}}, \operatorname{com} 1 \leq i \leq n,
$$

formam um conjunto completo de idempotentes de $\mathbb{F} G$, ou seja, dois a dois ortogonais tais que $e_{0}+e_{1}+\cdots+e_{n}=1$. 
Desejamos estender os resultados para $p$-grupos abelianos finitos. Dado um grupo $G$ nestas condições, para cada subgrupo cocíclico $H$ de $G$ vamos determinar um idempotente de $\mathbb{F} G$. Uma vez que $G / H$ é cíclico de ordem uma potência de um primo $p$, existe um único subgrupo $H_{1}$ de $G$ que contém $H$, tal que $\left[H_{1}: H\right]=p$. Definindo $e_{H}=\widehat{H}-\widehat{H_{1}}$, naturalmente $e_{H} \neq 0$.

O próximo resultado caracteriza quando estes idempotentes são primitivos

Teorema 1.21 ([6], Thm.4.1). Sejam p um primo impar e A um p-grupo abeliano de expoente $p^{r}$. Então o conjunto de idempotentes dados acima, é um conjunto de idempotentes primitivos em $\mathbb{F} G$ se, e somente se, são verificadas as seguintes condições:

(i) $p^{r}=4$ e $q \equiv 3(\bmod 4)$;

(ii) $o(q)=\varphi\left(p^{n}\right)$ em $U\left(\mathbb{Z}_{p^{n}}\right)$, onde $\varphi$ é a função de Euler.

Caso contrário, determinamos o número de componentes simples da álgebra semissimples $\mathbb{F} G$ via $q$-classes. Utilizamos o método do Descenso de Galois, para saber como estes idempotentes se decompõem em soma de primitivos. 


\section{Capítulo 2}

\section{Códigos em $\mathbb{F} G$}

O propósito deste Capítulo é estudar códigos em álgebras de grupo: com este intuito, vamos começar introduzindo algumas definições.

Definição 2.1. Um código linear sobre um corpo finito $\mathbb{F}$ é um subespaço próprio de $\mathbb{F}^{n}$, onde $n$ é inteiro positivo.

Se $G$ é um grupo com $n$ elementos então $\mathbb{F} G \cong \mathbb{F}^{n}$, como $\mathbb{F}$ espaços vetoriais. Casos particularmente interessantes de subespaços de $\mathbb{F} G$ são seus ideais.

Definição 2.2. Códigos em álgebra de grupo são ideais de $\mathbb{F} G$. Ainda, os ideais minimais de $\mathbb{F} G$ são chamados de códigos minimais.

Definição 2.3. Seja I um ideal de $\mathbb{F} G \operatorname{com} \alpha=\sum_{g \in G} \alpha_{g} g$ e $\beta=\sum_{g \in G} \beta_{g} g \in I$.

A distância de Hamming entre $\alpha$ e $\beta$ é definida por

$$
d(\alpha, \beta)=\left|\left\{g ; \alpha_{g} \neq \beta_{g}, g \in G\right\}\right|
$$

ou seja, é o número de elementos do suporte de $\alpha-\beta$.

O peso de a é o número de elementos de seu suporte, isto é,

$$
\omega(\alpha)=d(\alpha, 0)
$$

O peso do código de grupo I é

$$
\omega(I)=\min \{\omega(\alpha) ; \alpha \in I, \alpha \neq 0\}=\{d(\alpha-\beta) ; \alpha, \beta \in I, \alpha \neq \beta\},
$$

ou seja, a distância minima do código.

Chamamos de distribuição de peso de um código a função que para cada número inteiro $r$, $0 \leq r \leq n$, onde n é o comprimento do código, associa o número de palavras do código cujo peso é $r$.

Definiremos também os grupos que iremos trabalhar bem como mostraremos alguns resultados obtidos. Os grupos $G$ de interesse neste trabalho são finitos tais que $G / \mathscr{Z}(G) \cong C_{p} \times C_{p}$, onde $p$ é primo e $\mathscr{Z}(G)$ denota o centro do grupo $G$. Estes grupos primeiramente apareceram no trabalho de Edgar G. Goodaire [8] para $p=2$, quando estava estudando loops de Moufang cujo anel de loop é 
alternativo mas não é associativo. Desde então, eles têm aparecido em muitos outros contextos. Estes grupos estão explicitados no capítulo $V$ do livro Alternative Loop Rings [9], embasado no trabalho de G. Leal e C. Polcino Milies [15] no caso $p=2$. Para primos quaisquer foram classificados por M. G. Cornelissem e C. Polcino Milies [3]. Nosso objetivo é estudar os códigos oriundos deste tipo de grupos, sobre corpos finitos (de característica diferente de $p$ ) e procurar exemplos em que o peso seja o maior possível, para uma dimensão dada.

Definição 2.4. Um grupo é decomponivel se é o produto direto de dois subgrupos normais próprios não triviais. Se isto não ocorrer dizemos que tal grupo é indecomponivel.

O teorema da classificação destes grupos, no caso finito [3, Teorema 2.2], se traduz no seguinte:

Teorema 2.5. Seja $\mathscr{G}$ grupo finito tal que $\mathscr{G} / \mathscr{Z}(\mathscr{G}) \cong C_{p} \times C_{p}$ se, e somente se, pode-se escrever $\mathscr{G}$ da seguinte forma $\mathscr{G}=G \times A$ onde $A$ é um grupo abeliano e $G=<x, y, \mathscr{Z}(G)>$ é um p-grupo, indecomponivel não abeliano tal que:

- $\mathscr{Z}(G)=<t_{1}>\times<t_{2}>\times<t_{3}>$;

- $o\left(t_{i}\right)=p^{m_{i}}$ para $i=1,2,3, m_{1} \geq 1, m_{2}, m_{3} \geq 0$;

- $s=[x, y]=x^{-1} y^{-1} x y=t_{1}^{p^{m_{1}-1}} \in<t_{1}>$;

- $x^{p}, y^{p} \in \mathscr{Z}(G) \operatorname{com} x^{p} \in<t_{1}>\times<t_{2}>e y^{p} \in<t_{1}>\times<t_{2}>\times<t_{3}>$.

Isso mostra que o grupo indecomponível $G$, tem a propriedade

$$
\frac{G}{\mathscr{Z}(G)} \cong C_{p} \times C_{p} \quad \text { e } \quad|G|=p^{2}|\mathscr{Z}(G)| .
$$

Os grupos indecomponíveis finitos são de um dos seguintes tipos:

\begin{tabular}{|l|l|l|}
\hline Classe & \multicolumn{1}{|c|}{ Geradores } & \multicolumn{1}{|c|}{ Grupos } \\
\hline $\mathscr{D}_{1}$ & $x, y, t_{1}$ & $\left\langle x, y, t_{1} ; x^{p}=y^{p}=t_{1}^{p^{m_{1}}}=1\right\rangle, m_{1} \geq 1$ \\
\hline $\mathscr{D}_{2}$ & $x, y, t_{1}$ & $\left\langle x, y, t_{1} ; x^{p}=y^{p}=t_{1}, t_{1}^{p^{m_{1}}}=1\right\rangle, m_{1} \geq 1$ \\
\hline $\mathscr{D}_{3}$ & $x, y, t_{1}, t_{2}$ & $\left\langle x, y, t_{1}, t_{2} ; x^{p}=t_{1}^{p^{m} 1}=t_{2}^{p^{m}}=1, y^{p}=t_{2}\right\rangle, \quad m_{1}, m_{2} \geq 1$ \\
\hline $\mathscr{D}_{4}$ & $x, y, t_{1}, t_{2}$ & $\left\langle x, y, t_{1}, t_{2} ; x^{p}=t_{1}, y^{p}=t_{2}, t_{1}^{p^{m_{1}}}=t_{2}^{p^{m}}=1\right\rangle, m_{1}, m_{2} \geq 1$ \\
\hline $\mathscr{D}_{5}$ & $x, y, t_{1}, t_{2}, t_{3}$ & $\left\langle x, y, t_{1}, t_{2}, t_{3} ; x^{p}=t_{2}, y^{p}=t_{3}, t_{1}^{p^{m}}=t_{2}^{p^{m}}=t_{3}^{p^{m}}=1\right\rangle, m_{1}, m_{2}, m_{3} \geq 1$ \\
\hline
\end{tabular}

Os grupos $G$ da forma $\mathscr{D}_{i}(1 \leq i \leq 5)$, têm ordem uma potência de $p$, o centro é um produto direto de grupos cíclicos e $s=[x, y]=t_{1}^{p^{m_{1}-1}}\left(m_{1} \geq 1\right)$, que é um gerador do subgrupo comutador, denotado por $G^{\prime}$. Vamos mostrar que ele é central e cíclico de ordem $p$.

Lema 2.6. Seja $G$ um grupo tal que $G / \mathscr{Z}(G) \cong C_{p} \times C_{p}$. Então o subgrupo comutador $G^{\prime}$ é central e cíclico de ordem $p$.

\section{Demonstração:}

Por hipótese $G / \mathscr{Z}(G) \cong C_{p} \times C_{p}$, então existem $x, y \in G$ tais que

$$
G=\langle x, y, \mathscr{Z}(G)\rangle \operatorname{com} x^{p}, y^{p} \in \mathscr{Z}(G) .
$$

Note que, $\mathscr{Z}(G)$ é um subgrugo normal de $G$ e $G / \mathscr{Z}(G)$ é abeliano, donde $G^{\prime} \subset \mathscr{Z}(G)$. Com isto mostramos que $G^{\prime}$ é central.

Todo elemento $g \in G$ é da forma $g=x^{n} y^{m} z$, onde $x, y \in G$ e $z \in \mathscr{Z}(G)$. Logo,

$$
\left[g_{1}, g_{2}\right]=\left[x^{n} y^{m} z_{1}, x^{r} y^{t} z_{2}\right]=\left[x^{n} y^{m}, x^{r} y^{t}\right]
$$


Então,

$$
G^{\prime}=\left\langle\left\{\left[x^{n} y^{m}, x^{r} y^{t}\right] ; 0 \leq n, m, r, t<p\right\}\right\rangle .
$$

De maneira geral, se $x, y, z \in G$, então:

i) $[x, y]^{-1}=[y, x]$;

ii) $[x y, z]=[x, z]^{y}[y, z]$ e $[x, y z]=[x, z][x, y]^{z}$.

Aqui, expressão $x^{y}$ denota o conjugado de $x$ por $y$, definido por $y^{-1} x y$.

No nosso caso $G^{\prime} \subset \mathscr{Z}(G)$, e portanto

ii') $[x y, z]=[x, z][y, z]$ e $[x, y z]=[x, z][x, y]$.

Usando i) e ii'), provaremos que $G^{\prime}$ é cíclico de ordem $p$ :

- $G^{\prime}$ é cíclico:

$$
\begin{aligned}
{\left[x^{n} y^{m}, x^{r} y^{t}\right] } & =\left[x^{n}, x^{r} y^{t}\right]\left[y^{m}, x^{r} y^{t}\right] \\
& =\left[x^{n}, x^{r}\right]\left[x^{n}, y^{t}\right]\left[y^{m}, x^{r}\right]\left[y^{m}, y^{t}\right] \\
& =\left[x^{n}, y^{t}\right]\left[x^{r}, y^{m}\right]^{-1} \\
& =[x, y]^{n t}[x, y]^{-r m} \\
& =[x, y]^{n t-r m}
\end{aligned}
$$

- $G^{\prime}$ tem ordem $p$ :

Uma vez que $\left[x, y^{p}\right]=[x, y]^{p}=1$, isto implica que $\left|G^{\prime}\right|=p$.

Assim, denotando $[x, y]=s$ temos que $G^{\prime}=\left\{1, s, s^{2}, \ldots, s^{p-1}\right\}$.

As classes de conjugação de $G$ são da seguinte forma:

$$
\mathscr{C}_{a}=\{a\} \text {, se } a \in \mathscr{Z}(G) \text { e } \mathscr{C}_{a}=\left\{a, a s, a s^{2}, \ldots, a s^{p-1}\right\} \text {, se } a \notin \mathscr{Z}(G) .
$$

De fato, se $a \notin \mathscr{Z}(G)$, existe $g \in G$ tal que $[a, g] \neq 1 \mathrm{e}, a^{-1} g^{-1} a g \in G^{\prime}$ o que implica $a^{-1} g^{-1} a g=$ $s^{r}$, para algum $r \in\{1,2, \cdots, p-1\}$. Assim,

$$
\begin{aligned}
a^{-1} g^{-1} a g=s^{r} \Rightarrow g^{-1} a g & =a s^{r} \quad \text { conjugando novamente } \\
\Rightarrow g^{-1}\left(g^{-1} a g\right) g & =a s^{2 r} \\
\vdots & \\
\Rightarrow g^{-(p-1)} a g^{p-1} & =a s^{(p-1) r}
\end{aligned}
$$

o que implica $g^{-p} a g^{p}=a s^{p r}=a$ pois, $o(s)=p$.

Para qualquer outro $g_{1} \in G$ tal que $\left[a, g_{1}\right] \neq 1$, temos que $a^{-1} g_{1}^{-1} a g_{1} \in G^{\prime}$ o que implica $a^{-1} g_{1}^{-1} a g_{1}=s^{r_{1}}$, para algum $r_{1} \in\{1,2, \cdots, p-1\}$, que está listado acima.

Portanto, $\mathscr{C}_{a}=\left\{a, a s^{r}, a s^{2 r}, \cdots, a s^{(p-1) r}\right\}$, com $r \in\{1,2, \cdots, p-1\}$ de onde se conclui, reorganizando os elementos do conjunto caso seja necessário, $\mathscr{C}_{a}=\left\{a, a s, a s^{2}, \cdots, a s^{(p-1)}\right\}$.

Observação 2.7. Dizemos que um grupo G possui a Propriedade da Comutatividade Limitada, termo em inglês "Limited Commutativity" denotada por LC, se G não é comutativo e se, para qualquer par de elementos $x, y \in G$, tem-se que $x y=y x$ se, e somente se, pelo menos um dos elementos $x, y$ ou xy pertence a $\mathscr{Z}(G)$. 
Os grupos que possuem a propriedade LC e um único comutador não trivial s (em cujo caso necessariamente $p=2$ ), são chamados de SLC grupos, e aos códigos com base neles $\boldsymbol{S L C}$-Códigos.

A partir de agora, indicaremos por $G$ um dos cinco tipos de grupos indecomponíveis finitos descritos acima.

Um código é dito central, quando é gerado por um idempotente central, isto é, quando o ideal correspondente é bilateral. No que segue mostraremos que o código central de $\mathbb{F} G$ é combinatoriamente equivalente a um abeliano.

\subsection{Estrutura dos Códigos Centrais}

Nesta seção, veremos que existe uma relação entre os códigos centrais de $\mathbb{F} G$ e certos códigos abelianos em $\mathbb{F}\left(C_{p} \times C_{p} \times \mathscr{Z}(G)\right)$. Os resultados aqui descritos foram inspirados em [19].

Definição 2.8. Sejam $G$ e $\tilde{G}$ dois grupos finitos de mesma ordem, e $\mathbb{F}$ um corpo finito. Sejam $\mathbb{F} G$ e $\mathbb{F} \tilde{G}$ as álgebras de grupo correspondentes. Uma equivalência combinatória é um $\mathbb{F}$-isomorfismo de espaços vetoriais $\gamma: \mathbb{F} G \rightarrow \mathbb{F} \tilde{G}$ induzido por uma bijeção $\gamma: G \rightarrow \tilde{G}$. Os códigos $\mathscr{C}$ em $\mathbb{F} G$ e $\tilde{\mathscr{C}} \subseteq \mathbb{F} \tilde{G}$ são ditos combinatoriamente equivalentes se existe uma equivalência combinatória $\gamma: \mathbb{F} G \rightarrow \mathbb{F} \tilde{G}$ tal que $\gamma(\mathscr{C})=\tilde{\mathscr{C}}$.

Certamente códigos combinatoriamente equivalentes devem ter as mesmas distribuições de peso.

Dado $G=\langle x, y, \mathscr{Z}(G)\rangle$, conforme Teorema 2.5, seja $\tilde{G}=\langle\tilde{x}\rangle \times\langle\tilde{y}\rangle \times \mathscr{Z}(G)$ onde $o(\tilde{x})=o(\tilde{y})=p$. Definimos a bijeção $\gamma: G \rightarrow \tilde{G}$ por $\gamma\left(x^{i} y^{j} z\right)=\tilde{x}^{i} \tilde{y}^{j} z$, com $i, j \in\{0,1, \ldots, p-1\}$. Como $G$ é base de $\mathbb{F} G$ e $\tilde{G}$ é base de $\mathbb{F} \tilde{G}, \gamma$ é um $\mathbb{F}$-isomorfismo de espaços vetoriais. Logo, $\gamma: \mathbb{F} G \rightarrow \mathbb{F}\left(C_{p} \times C_{p} \times \mathscr{Z}(G)\right)$ é uma equivalência combinatória. Além disso, o seguinte resultado é válido

Lema 2.9. Sejam $G$ um grupo e $\gamma$ uma função como definidos anteriormente.

Se $c \in \mathscr{Z}(\mathbb{F} G)$, então para todo $\alpha \in \mathbb{F} G$ temos $\gamma(\alpha \cdot c)=\gamma(\alpha) \cdot \gamma(c)$.

\section{Demonstração:}

Seja $c=\sum_{j=0}^{p-1}\left(\sum_{i=0}^{p-1} f_{i j} x^{i}\right) y^{j} z \in \mathscr{Z}(\mathbb{F} G)$, com $z \in \mathscr{Z}(G)$.

Dado $\alpha=\left(\sum_{l=0}^{p-1}\left(\sum_{k=0}^{p-1} \alpha_{k l} x^{k}\right) y^{l}\right) z_{1} \in \mathbb{F} G$, com $z_{1} \in \mathscr{Z}(G)$,

basta mostrar que $\gamma\left(x^{k} y^{l} z_{1} \cdot c\right)=\gamma\left(x^{k} y^{l} z_{1}\right) \gamma(c)$, para todo $k, l \in\{0,1, \ldots, p-1\}$.

Pois, $\gamma(\alpha \cdot c)=\gamma\left(\left(\sum_{k, l=0}^{p-1} \alpha_{k l} x^{k} y^{l}\right) z_{1} \cdot c\right)=\sum_{k, l=0}^{p-1} \alpha_{k l}\left(\gamma\left(x^{k} y^{l} z_{1} \cdot c\right)\right)$.

Como $c$ é central, $\gamma\left(x^{k} y^{l} z_{1} \cdot c\right)=\gamma\left(x^{k} \cdot c \cdot y^{l} z_{1}\right)$ e para $k, l \in\{0,1, \ldots, p-1\}$ fixados, temos

$$
\begin{aligned}
\gamma\left(x^{k} \cdot c \cdot y^{l} z_{1}\right) & =\gamma\left(x^{k}\left(\sum_{j=0}^{p-1}\left(\sum_{i=0}^{p-1} f_{i j} x^{i}\right) y^{j} z\right) y^{l} z_{1}\right) \\
& =\gamma\left(\sum_{j=1}^{p-1}\left(\sum_{i=0}^{p-1} f_{i j} x^{k+i}\right) y^{l+j} z z_{1}\right) \\
& =\sum_{j=0}^{p-1}\left(\sum_{i=0}^{p-1} f_{i j} \tilde{x}^{k+i}\right) \tilde{y}^{l+j} z z_{1} \\
& =\tilde{x}^{k} \tilde{y}^{l} z_{1} \sum_{j=0}^{p-1}\left(\sum_{i=0}^{p-1} f_{i j} \tilde{x}^{i}\right) \tilde{y}^{j} z \\
& =\gamma\left(x^{k} y^{l} z_{1}\right) \cdot \gamma(c) .
\end{aligned}
$$


Portanto, para qualquer $\alpha \in \mathbb{F} G$ e $c \in \mathscr{Z}(\mathbb{F} G)$, pela linearidade de $\gamma$, temos $\gamma(\alpha \cdot c)=\gamma(\alpha) \cdot \gamma(c)$.

Além disso, dado o gerador idempotente $e \in \mathscr{Z}(\mathbb{F} G)$ de $\mathscr{C}$, segue que $\gamma(e)$ é o gerador idempotente de $\gamma(\mathscr{C})$, conforme pode ser visto na proposição que segue:

Proposição 2.10. Sejam $G$ um grupo tal que $G / \mathscr{Z}(G) \cong C_{p} \times C_{p}$.

Se e o gerador idempotente central de $\mathscr{C} \subset \mathbb{F} G$ então, $\gamma(\boldsymbol{e})$ é o gerador idempotente de $\gamma(\mathscr{C})$.

\section{Demonstração:}

Seja e o único gerador idempotente de $\mathscr{C}$, isto é $\mathscr{C}=\{\alpha \cdot e ; \alpha \in \mathbb{F} G\}=\langle e\rangle$. Assim,

$$
\begin{aligned}
\gamma(\mathscr{C}) & =\{\gamma(\alpha \cdot e) ; \alpha \in \mathbb{F} G\}, \\
& =\{\gamma(\alpha) \cdot \gamma(e) ; \alpha \in \mathbb{F} G\}, \text { pelo Lema } 2.9 \\
& =\left\{\tilde{\alpha} \cdot \gamma(e) ; \tilde{\alpha} \in \mathbb{F}\left(C_{p} \times C_{p} \times \mathscr{Z}(G)\right)\right\} \\
& =\langle\gamma(e)\rangle,
\end{aligned}
$$

o que nos diz que $\gamma(\mathbf{e})$ é o gerador de $\gamma(\mathscr{C})$.

Vamos mostrar que $\gamma(e)$ é idempotente. Pelo Lema 2.9 segue que:

$$
\gamma(e)=\gamma(e \cdot e)=\gamma(e) \cdot \gamma(e)=\gamma(e)^{2} .
$$

Agora obtemos o resultado mais importante desta seção para $G$ um grupo tal que $G / \mathscr{Z}(G) \cong$ $C_{p} \times C_{p}$. Os resultados acima mostram que os códigos centrais de $\mathbb{F} G$ são combinatoriamente equivalentes a códigos abelianos, isto é, vale o seguinte teorema:

Teorema 2.11. Sejam $G$ e $\gamma$ como definidos anteriormente. Se $\mathscr{C}$ é um código central de $\mathbb{F} G$, então $\gamma(\mathscr{C})=\tilde{\mathscr{C}}$ é um código na álgebra comutativa $\mathbb{F}\left(C_{p} \times C_{p} \times \mathscr{Z}(G)\right)$.

Assim, nosso interesse é estudar os códigos não centrais da álgebra $\mathbb{F} G$, ou seja, os ideais laterais.

\subsection{Códigos Laterais em $\mathbb{F} G$}

Nosso objetivo inicial é determinar códigos minimais laterais de $\mathbb{F} G$, onde $G$ é um dos 5 tipos mencionados anteriormente. Para tal, é preciso encontrar os idempotentes primitivos centrais geradores das respectivas componentes simples, da parte não comutativa, desta álgebra. Para começar, vamos investigar a estrutura da parte não comutativa de $\mathbb{F} G$.

Da Proposição 1.5 temos

$$
\mathbb{F} G=\mathbb{F} G e_{G^{\prime}} \oplus \mathbb{F} G\left(1-e_{G^{\prime}}\right)
$$

onde $\mathbb{F} G e_{G^{\prime}} \cong \mathbb{F}\left(G / G^{\prime}\right)$ é a soma de todas as componentes simples comutativas, onde $G^{\prime}=$ $\left\{1, s, \ldots, s^{p-1}\right\}$ e $e_{G^{\prime}}=\widehat{G^{\prime}}$. 
Como $\mathbb{F} G$ é semissimples, $\mathbb{F} G$ possui o mesmo número de componentes simples do $\mathscr{Z}(\mathbb{F} G)$. Então,

$$
\mathscr{Z}(\mathbb{F} G)=\mathscr{Z}(\mathbb{F} G) e_{G^{\prime}} \oplus \mathscr{Z}(\mathbb{F} G)\left(1-e_{G^{\prime}}\right) \cong \mathbb{F}\left(G / G^{\prime}\right) \oplus \mathscr{Z}(\mathbb{F} G)\left(1-e_{G^{\prime}}\right) .
$$

Para o que desejamos fazer, usaremos um argumento de dimensão.

Lema 2.12. Sejam $G$ um grupo com $n$ elementos e $G^{\prime}$ seu subgrupo comutador com $p$ elementos, tal que $\frac{G}{\mathscr{Z}(G)} \cong C_{p} \times C_{p}$. A dimensão de $\mathscr{Z}(\mathbb{F} G)\left(1-e_{G^{\prime}}\right)$ é $\frac{n(p-1)}{p^{3}}$.

\section{Demonstração:}

Pela Proposição 1.5, $\operatorname{dim}\left(\mathbb{F}\left(G / G^{\prime}\right)\right)=\frac{n}{p}$.

Conforme a Definição 1.9 e o Teorema 1.10, recordemos que se $a \in \mathscr{Z}(G)$ as classes de conjugação são unitárias $\{a\}$; se $a \notin \mathscr{Z}(G)$ são da forma $\left\{a, a s, \ldots, a s^{p-1}\right\}$. A ordem de $\mathscr{Z}(G)$ é $\frac{n}{p^{2}}$, e este é o número das classes unitárias. Assim, o número de elementos que não pertencem a $\mathscr{Z}(G)$ é $n-\frac{n}{p^{2}}=\frac{n\left(p^{2}-1\right)}{p^{2}}$, e cada classe possui $p$ elementos. Portanto, temos $\frac{n\left(p^{2}-1\right)}{p^{3}}$ classes de elementos que não pertencem a $\mathscr{Z}(G)$. Segue que

$$
\operatorname{dim}[\mathscr{Z}(\mathbb{F} G)]=\frac{n}{p^{2}}+\frac{n\left(p^{2}-1\right)}{p^{3}}=\frac{n\left(p^{2}+p-1\right)}{p^{3}} .
$$

Por $(2.1), \frac{n\left(p^{2}+p-1\right)}{p^{3}}-\frac{n}{p}=\frac{n(p-1)}{p^{3}}$ é a dimensão $\mathscr{Z}(\mathbb{F} G)\left(1-e_{G^{\prime}}\right)$, como queríamos mostrar.

Da Proposição 1.4 e como $e_{G^{\prime}} \in \mathbb{F}(\mathscr{Z}(G))$ temos:

$$
\mathbb{F}(\mathscr{Z}(G))=\mathbb{F}(\mathscr{Z}(G)) e_{G^{\prime}} \oplus \mathbb{F}(\mathscr{Z}(G))\left(1-e_{G^{\prime}}\right)
$$

$\operatorname{com} \mathbb{F}(\mathscr{Z}(G)) e_{G^{\prime}} \cong \mathbb{F}\left(\mathscr{Z}(G) / G^{\prime}\right)$.

Lema 2.13. Sejam $G$ um grupo com $n$ elementos e $G^{\prime}$ seu subgrupo comutador com $p$ elementos, tal que $\frac{G}{\mathscr{Z}(G)} \cong C_{p} \times C_{p}$. A dimensão de $\mathbb{F}(\mathscr{Z}(G))\left(1-e_{G^{\prime}}\right)$ é $\frac{n(p-1)}{p^{3}}$.

\section{Demonstração:}

Pelo exposto em 2.2:

$$
\begin{aligned}
\operatorname{dim}\left[\mathbb{F}(\mathscr{Z}(G))\left(1-e_{G^{\prime}}\right)\right] & =|\mathscr{Z}(G)|-\frac{|\mathscr{Z}(G)|}{\left|G^{\prime}\right|} \\
& =\frac{n}{p^{2}}-\frac{n}{p^{3}} \\
& =\frac{n(p-1)}{p^{3}},
\end{aligned}
$$

como queríamos mostrar.

Naturalmente,

$$
\mathbb{F}(\mathscr{Z}(G))\left(1-e_{G^{\prime}}\right) \subset \mathscr{Z}(\mathbb{F} G)\left(1-e_{G^{\prime}}\right),
$$

e dos Lemas 2.12 e 2.13 , resulta a seguinte 
Proposição 2.14. Seja $G$ um grupo tal que $G / \mathscr{Z}(G) \cong C_{p} \times C_{p}$,

$$
\mathscr{Z}\left(\mathbb{F} G\left(1-e_{G^{\prime}}\right)\right)=\mathbb{F}(\mathscr{Z}(G))\left(1-e_{G^{\prime}}\right) .
$$

Corolário 2.15. Seja $G$ um grupo tal que $G / \mathscr{Z}(G) \cong C_{p} \times C_{p}$, $\mathscr{Z}\left(\mathbb{F} G\left(1-e_{G^{\prime}}\right)\right)$ e $\mathbb{F}(\mathscr{Z}(G))\left(1-e_{G^{\prime}}\right)$ possuem os mesmos idempotentes primitivos.

Assim,

$$
\mathscr{Z}(\mathbb{F} G)\left(1-e_{G^{\prime}}\right)=\left\{\alpha\left(1-e_{G^{\prime}}\right) ; \alpha \in \mathscr{Z}(\mathbb{F} G)\right\} .
$$

Sejam $\alpha \in \mathscr{Z}(\mathbb{F} G)$ e $\mathscr{B}$ um conjunto completo das classes de conjugação não unitárias. Então pelo Teorema 1.10:

$$
\begin{aligned}
\alpha & =\sum_{g \in \mathscr{Z}(G)} a_{g} g+\sum_{h \in \mathscr{B}} b_{h}\left(h+h s+\cdots+h s^{p-1}\right) \\
& =\sum_{g \in \mathscr{Z}(G)} a_{g} g+\sum_{h \in \mathscr{B}} b_{h} h\left(p e_{G^{\prime}}\right) .
\end{aligned}
$$

Então,

$$
\alpha \in \mathscr{Z}(\mathbb{F} G)\left(1-e_{G^{\prime}}\right) \text { se, e somente se, }\left(1-e_{G^{\prime}}\right) \alpha=\left(1-e_{G^{\prime}}\right) \sum_{g \in \mathscr{Z}(G)} a_{g} g
$$

pois, para cada $h$ temos $\left(1-e_{G^{\prime}}\right) p e_{G^{\prime}}=0$.

Concluímos que, o número de componentes simples de $\mathbb{F} G\left(1-e_{G^{\prime}}\right)$ é o número de componentes simples de $\mathbb{F}(\mathscr{Z}(G))$, que é o número de $q$-classes de $\mathscr{Z}(G)$, menos o número de componentes simples de $\mathbb{F}\left(\frac{\mathscr{Z}(G)}{G^{\prime}}\right)$, que é o número de $q$-classes de $\frac{\mathscr{Z}(G)}{G^{\prime}}$. Portanto, o resultado é válido

Proposição 2.16. O número de componentes simples da parte não comutativa de $\mathbb{F} G$ é igual ao número de q-classes do $\mathscr{Z}(G)$ menos o número de q-classes de $\frac{\mathscr{Z}(G)}{G^{\prime}}$.

Mostraremos que a parte não comutativa da álgebra de grupo $\mathbb{F} G$ é isomorfa a uma soma direta de álgebras de matrizes $p \times p$, sobre corpos finitos. Para a demonstração deste resultado, vamos relembrar alguns conceitos, do nosso caso:

- $\mathbb{F} G$ é semissimples e por Wedderburn-Artin $\mathbb{F} G \cong \oplus_{i=1}^{s} M_{n_{i}}\left(\mathbb{F}_{i}\right)$, com $\mathbb{F}_{i}$ uma extensão de $\mathbb{F}$.

- Seja $\rho_{i}: G \rightarrow M_{n_{i}}\left(\mathbb{F}_{i}\right)$ a projeção na $i$-ésima componente.

Então $\{\rho(g) ; g \in G\}$ é um conjunto de geradores de $M_{n_{i}}\left(\mathbb{F}_{i}\right)$. O que implica, se $z \in \mathscr{Z}(G)$ então $\rho(z)$ é central em $M_{n_{i}}\left(\mathbb{F}_{i}\right)$, isto é, $\rho(z)=\alpha I$, com $\alpha \in \mathbb{F}_{i}$ e $I$ a matriz identidade de ordem $n_{i}$. Seja $\left\{g_{1}, g_{2}, \cdots, g_{t}\right\}$ um conjunto de representantes das classes laterais de $\mathscr{Z}(G)$ em $G$. Dado $g \in G$, temos que $\rho(g)=\rho\left(g_{i} z\right)$, para algum $z \in \mathscr{Z}(G)$ e $1 \leq i \leq t$, donde

$$
\rho(g)=\rho\left(g_{i}\right) \alpha, \operatorname{com} \alpha \in \mathbb{F}_{i} .
$$


Consequentemente, $\left\{\rho\left(g_{1}\right), \rho\left(g_{2}\right), \cdots, \rho\left(g_{t}\right)\right\}$ é um conjunto de geradores de $M_{n_{i}}\left(\mathbb{F}_{i}\right)$ sobre $\mathbb{F}_{i}$, de dimensão $n_{i}^{2}$. Então $n_{i}^{2} \leq t=[G: \mathscr{Z}(G)]=p^{2}$.

Teorema 2.17. Seja $G$ tal que $G / \mathscr{Z}(G) \cong C_{p} \times C_{p}$, então $\mathbb{F} G\left(1-e_{G^{\prime}}\right) \cong \oplus_{i=1}^{k} M_{p}\left(\mathbb{F}_{i}\right)$, onde $k$ é o número de componentes simples não comutativas de $\mathbb{F} G$.

\section{Demonstração:}

Pela Proposição 1.5 e pelo Lema 2.12, temos que

$$
\operatorname{dim}\left(\mathbb{F} G\left(1-e_{G^{\prime}}\right)\right)=\frac{n(p-1)}{p} \text { e } \operatorname{dim}\left(\mathscr{Z}\left(\mathbb{F} G\left(1-e_{G^{\prime}}\right)\right)\right)=\frac{n(p-1)}{p^{3}} .
$$

Sabemos também $\operatorname{dim}\left(\oplus_{i=1}^{k} M_{n_{i}}\left(\mathbb{F}_{i}\right)\right)=\sum_{i=1}^{k} n_{i}^{2} \cdot \operatorname{dim}\left(\mathbb{F}_{i}\right)$.

Em $M_{n_{i}}\left(F_{i}\right)$ cada $n_{i} \geq 2$, pois se algum $n_{i}$ fosse igual a 1 , estaria na parte comutativa de $\mathbb{F} G$.

Vamos mostrar que $n_{i}=p, 1 \leq i \leq k$.

$\operatorname{De} \operatorname{dim}\left(\mathbb{F} G\left(1-e_{G^{\prime}}\right)\right)=\sum_{i=1}^{k} n_{i}^{2} \cdot \operatorname{dim}\left(\mathbb{F}_{i}\right)=\frac{n(p-1)}{p} \mathrm{e}$

$$
\begin{gathered}
\mathscr{Z}\left(\oplus_{i=1}^{k} M_{n_{i}}\left(F_{i}\right)\right) \cong \oplus_{i=1}^{k} F_{i} \text { com } \\
\operatorname{dim}\left(\mathscr{Z}\left(\oplus_{i=1}^{k} M_{n_{i}}\left(F_{i}\right)\right)\right)=\sum_{i=1}^{k} \operatorname{dim}\left(\mathbb{F}_{i}\right)=\frac{n(p-1)}{p^{3}},
\end{gathered}
$$

isto é,

$$
\sum_{i=1}^{k} n_{i}^{2} \cdot \operatorname{dim}\left(\mathbb{F}_{i}\right)=p^{2} \cdot \sum_{i=1}^{k} \operatorname{dim}\left(\mathbb{F}_{i}\right) .
$$

Então, de $n_{i}^{2} \leqslant[G: \mathscr{Z}(G)]=p^{2}$, segue $n_{i}=p, 1 \leq i \leq k$.

Portanto, a parte não comutativa das componentes simples de $\mathbb{F} G$ é isomorfa à soma direta de álgebras de matrizes $p \times p$ com entradas em corpos finitos.

No capítulo que segue, determinaremos os ideais à esquerda na álgebra das matrizes sobre um corpo finito. Em particular, determinaremos os idempotentes primitivos em $M_{n}\left(\mathbb{F}_{q}\right)$, o anel das matrizes $n \times n$ sobre $\mathbb{F}_{q}$, os quais geram os ideais minimais laterais, ou seja, os códigos minimais laterais em $\mathbb{F} G$. 


\section{Capítulo 3}

\section{Ideais à esquerda em $M_{n}\left(\mathbb{F}_{q}\right)$}

Uma vez que $M_{n}\left(\mathbb{F}_{q}\right)$ é semissimples, todo ideal à esquerda é gerado por um elemento. Em particular, todo ideal à esquerda tem um gerador que é idempotente. Nesta seção, computamos o número de ideais à esquerda de tal anel, o número de idempotentes diferentes que geram o mesmo ideal à esquerda, e damos explicitamente um conjunto de geradores de um ideal à esquerda de um determinado posto $k$, com $1 \leq k \leq n$.

Dada $M \in M_{n}\left(\mathbb{F}_{q}\right)$, denotamos por $W(M)$ o subespaço de $\mathbb{F}_{q}^{n}$ gerado pelas linhas de $M$. Além disso, usamos a notação $\langle M\rangle$ para o ideal à esquerda gerado pela matriz $M$. Note que, visto que

$$
\operatorname{posto}(A M) \leq \operatorname{posto}(M), \text { para todo } A \in M_{n}\left(\mathbb{F}_{q}\right) .
$$

Consequentemente, dois geradores do mesmo ideal tem o mesmo posto e

$$
\operatorname{posto}(M)=\sup \left\{\operatorname{posto}(X M) ; X \in M_{n}\left(\mathbb{F}_{q}\right)\right\} .
$$

Para um ideal $J=\langle M\rangle$ definimos $\operatorname{posto}(J)=\operatorname{posto}(M)$.

Os resultados aqui obtidos são referentes a ideais à esquerda, mas similarmente são válidos para ideais à direita.

\subsection{Ideais e subespaços}

Pretendemos contar o número de ideais à esquerda de $M_{n}\left(\mathbb{F}_{q}\right)$. Para tal vamos estabelecer uma correspondência destes ideais com subespaços de $\mathbb{F}_{q}^{n}$. Começaremos com o que segue:

Lema 3.1. Dadas $M_{1}, M_{2} \in M_{n}\left(\mathbb{F}_{q}\right)$, então $\left\langle M_{1}\right\rangle \subset\left\langle M_{2}\right\rangle$ se, e somente se, as linhas de $M_{1}$ são combinações lineares das linhas de $M_{2}$.

\section{Demonstração:}

Dadas $M_{1}=\left(a_{i j}\right)$ e $M_{2}=\left(b_{i j}\right) \in M_{n}\left(\mathbb{F}_{q}\right)$, note que $\left\langle M_{1}\right\rangle \subset\left\langle M_{2}\right\rangle$ se, e somente se, $M_{1} \in\left\langle M_{2}\right\rangle$, isto é, se existe $\left(c_{i j}\right) \in M_{n}\left(\mathbb{F}_{q}\right)$, tal que $M_{1}=\left(c_{i j}\right) M_{2}$. 
Consequentemente, $\left(a_{i j}\right)=\sum_{k=1}^{n} c_{i k} b_{k j}, \forall i, j$ e a $l$-ésima linha de $M_{1}$ é

$$
\begin{aligned}
\left(a_{l 1}, a_{l 2}, \ldots, a_{l n}\right) & =\left(\sum_{k=1}^{n} c_{l k} b_{k 1}, \sum_{k=1}^{n} c_{l k} b_{k 2}, \ldots, \sum_{k=1}^{n} c_{l k} b_{k n}\right) \\
& =\sum_{k=1}^{n}\left(c_{l k} b_{k 1}, c_{l k} b_{k 2}, \ldots, c_{l k} b_{k n}\right) \\
& =\sum_{k=1}^{n} c_{l k}\left(b_{k 1}, b_{k 2}, \ldots, b_{k n}\right) \\
& =\sum_{k=1}^{n} c_{l k} L_{k}
\end{aligned}
$$

onde $L_{k}$ é a $k$-ésima linha de $M_{2}$.

A recíproca é imediatamente.

Consequentente, o seguinte resultado é válido

\section{Corolário 3.2.}

$$
\begin{aligned}
& \left\langle M_{1}\right\rangle \varsubsetneqq\left\langle M_{2}\right\rangle \text { se, e somente se, } W\left(M_{1}\right) \varsubsetneqq W\left(M_{2}\right) \\
& \left\langle M_{1}\right\rangle=\left\langle M_{2}\right\rangle \text { se, e somente se, } W\left(M_{1}\right)=W\left(M_{2}\right) .
\end{aligned}
$$

Desde que todo subespaço de $\mathbb{F}_{q}^{n}$ é da forma $W(M)$, para alguma $M \in M_{n}\left(\mathbb{F}_{q}\right)$, temos o que segue

Teorema 3.3. Dado um inteiro positivo $k, 1 \leq k \leq n-1$, existe uma bijeção $\varphi$ entre os subespaços de dimensão $k$ de $\mathbb{F}_{q}^{n}$, e o conjunto de ideais à esquerda de posto $k$ de $M_{n}\left(\mathbb{F}_{q}\right)$, como segue:

$\varphi:\left\{\right.$ Subespaços de dimensão $k$ de $\left.\mathbb{F}_{q}^{n}\right\} \rightarrow\left\{\right.$ Ideais à esquerda de posto $k$ de $\left.M_{n}\left(\mathbb{F}_{q}\right)\right\}$

$$
W\left(N_{i}\right) \mapsto\left\langle N_{i}\right\rangle .
$$

\section{Demonstração:}

Um subespaço $W k$-dimensional de um espaço vetorial $\mathbb{F}_{q}^{n}$ é determinado por uma base com $k$ elementos de $\mathbb{F}_{q}^{n}$, isto é, $k$ vetores linearmente independentes

$$
B=\left\{v_{1}, v_{2}, \cdots, v_{k}\right\}
$$

de $\mathbb{F}_{q}^{n}$. Considere a matriz $M$, cujas primeiras $k$ linhas são os vetores $v_{1}, v_{2}, \cdots, v_{k}$ e as demais linhas são combinações lineares destas. Claramente $W$ é igual a $W(M)$. Também, $W\left(M_{1}\right)=W\left(M_{2}\right)$, então $\left\langle M_{1}\right\rangle=\left\langle M_{2}\right\rangle$, pelo Corolário 3.2. Sendo assim, concluímos que $\varphi$ está bem definida. Além disso, como $\left\langle M_{1}\right\rangle=\left\langle M_{2}\right\rangle$ implica $W\left(M_{1}\right)=W\left(M_{2}\right)$, segue que $\varphi$ é injetora. Como todo ideal de $M_{n}\left(\mathbb{F}_{q}\right)$ é principal, $\varphi$ também é sobrejetora, e o resultado está provado. 
Em [16, cap. 24] está descrito o número de $k$-subespaços de $\mathbb{F}_{q}^{n}$, com $0<k \leqslant n$. Portanto, o seguinte teorema é válido

Teorema 3.4. O número de ideais à esquerda de posto $k$ em $M_{n}\left(\mathbb{F}_{q}\right)$ é

$$
\frac{\left(q^{n}-1\right)\left(q^{n-1}-1\right)\left(q^{n-2}-1\right) \cdots\left(q^{n-k+1}-1\right)}{\left(q^{k}-1\right)\left(q^{k-1}-1\right)\left(q^{k-2}-1\right) \cdots(q-1)} .
$$

Determinamos também condições para que duas matrizes idempotentes gerem o mesmo ideal:

Teorema 3.5. Dadas $R, S \in M_{n}\left(\mathbb{F}_{q}\right)$, são equivalentes:

(i) $R$ e $S$ idempotentes $e\langle S\rangle=\langle R\rangle$;

(ii) $R S=R$ e $S R=S$;

(iii) $R=S+(I-S) R S$ e $S$ é idempotente, onde $I$ denota a matriz identidade de $M_{n}\left(\mathbb{F}_{q}\right)$.

\section{Demonstração:}

(i) $\Rightarrow($ ii $)$

Se $R \in\langle S\rangle$ então, existe $A \in M_{n}\left(\mathbb{F}_{q}\right)$, tal que, $R=A S$.

Assim, $R S=A S \cdot S=A S=R$, pois $S$ é idempotente.

Similarmente, concluímos que $S R=S$.

$(i i) \Rightarrow(i)$

Note que (ii) implica que $R \in\langle S\rangle$ e $S \in\langle R\rangle$. Portanto, $\langle R\rangle=\langle S\rangle$.

Agora vejamos que ambas são idempotentes. De fato,

$$
\begin{gathered}
S^{2}=S R \cdot S=S \cdot R S=S R=S \\
\text { e } \\
R^{2}=R S \cdot R=R \cdot S R=R S=R .
\end{gathered}
$$

$($ ii $) \Rightarrow($ iii $)$

Calculando

$$
S+(I-S) R S \underbrace{=}_{R S=R} S+(I-S) R=S+R-S R \underbrace{=}_{S R=S} R .
$$

Além disso, $S^{2}=S R \cdot S=S \cdot R S=S R=S$

$$
\begin{gathered}
(i i i) \Rightarrow(\text { ii }) \quad \\
S R=S(S+(I-S) R S)=S, \\
\mathrm{e} \\
R=S+(I-S) R S=S+R S-\underbrace{S R}_{S} S=R S .
\end{gathered}
$$

No que segue, dado um idempotente determinamos todos os outros idempotentes que geram o mesmo ideal.

Teorema 3.6. Seja $S \in M_{n}\left(\mathbb{F}_{q}\right)$ um idempotente.

Então, para todo $M \in M_{n}\left(\mathbb{F}_{q}\right)$, a matriz $M_{0}=S+(I-S) M S$ é também um idempotente gerador de $\langle S\rangle$. Reciprocamente, todo idempotente gerador de $\langle S\rangle$ é desta forma. 


\section{Demonstração:}

Claramente $M_{0}$ é idempotente, e pela parte (ii) do Teorema 3.5, segue que

$$
\begin{gathered}
S M_{0}=S\left(S+\left(I d_{n}-S\right) M S\right)=S \text { o que implica que } S \in\left\langle M_{0}\right\rangle \quad \text { e } \\
M_{0} S=\left(S+\left(I d_{n}-S\right) M S\right) S=S+\left(I d_{n}-S\right) M S=M_{0} \text { o que implica que } M_{0} \in\langle S\rangle .
\end{gathered}
$$

Portanto, $\langle S\rangle=\left\langle M_{0}\right\rangle$.

A recíproca, segue imediatamente da parte (iii) do Teorema 3.5.

Observação 3.7. Se denotarmos por $[M]$ o ideal à direita gerado pela matriz $M \in M_{n}\left(\mathbb{F}_{q}\right)$ e por $V(M)$ o subespaço de $\mathbb{F}_{q}^{n}$ gerado pelas colunas de $M$, então valem os seguintes resultados análogos: (i) $\quad\left[M_{1}\right]=\left[M_{2}\right]$ se, e somente se, $V\left(M_{1}\right)=V\left(M_{2}\right)$;

(ii) $S e R, S \in M_{n}\left(\mathbb{F}_{q}\right)$ são idempotentes, então $[R]=[S]$ se, e somente se, $R S=S$ e $S R=R$.

\subsection{Idempotentes geradores de ideais à esquerda}

O propósito desta seção é exibir os idempotentes geradores de ideais à esquerda de posto $k$, em $M_{n}\left(\mathbb{F}_{q}\right)$ e determinar, para cada ideal $J$, o conjunto de todos os idempotentes geradores de $J$. Primeiramente, vamos estudar os idempotentes de posto 1.

Note que, pelo Teorema 3.4 aplicado ao caso em que $k=1$, temos o seguinte:

Lema 3.8. O número de ideais minimais do anel de matrizes $M_{n}\left(\mathbb{F}_{q}\right)$ é $\frac{q^{n}-1}{q-1}$.

Teorema 3.9. Os $\frac{q^{n}-1}{q-1}$ ideais minimais do anel das matrizes $M_{n}\left(\mathbb{F}_{q}\right)$, à esquerda, são gerados pelos idempotentes:

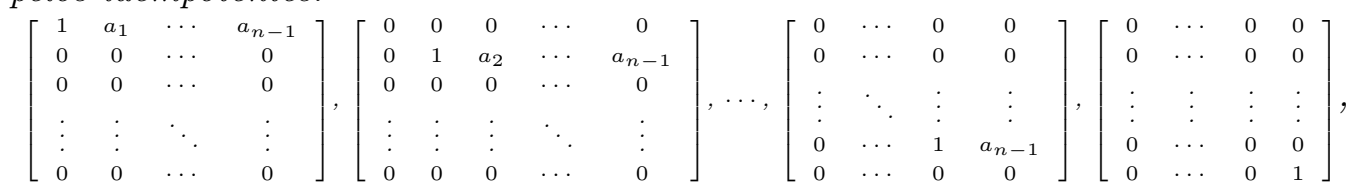

onde $a_{i}, 1 \leq i \leq n-1$, são elementos arbitrários de $\mathbb{F}_{q}$.

E cada ideal minimal à esquerda tem $q^{n-1}$ idempotentes geradores diferentes.

\section{Demonstração:}

Observamos que, de acordo com o Teorema 3.3, cada ideal minimal à esquerda $J=\langle M\rangle \in$ $M_{n}\left(\mathbb{F}_{q}\right)$ é relacionado com um subespaço $W=W(M) \subset \mathbb{F}_{q}^{n}$ de dimensão 1 . Seja $v=\left(a_{1}, a_{2}, \cdots, a_{n}\right)$ um gerador de $W$. Então, as linhas de qualquer matriz no ideal $J$ são múltiplos escalares de $v$.

Seja $i$ o menor índice tal que $a_{i} \neq 0,1 \leq i \leq n$, e consideremos o vetor $u=\frac{1}{a_{i}} v$. Segue que, $u=\left(0,0, \cdots, 0,1, b_{i+1}, \ldots, b_{n}\right)$, é também um gerador de $W$. Assim, podemos assumir que $J$ é gerado por uma matriz, da qual, a $i$-ésima linha é o vetor $u$ e as outras são nulas. Note que, matrizes diferentes do conjunto do enunciado geram ideais minimais, que são diferentes dois a dois.

Para provar a segunda parte do enunciado, seja $E_{11}$ a matriz cuja entrada na posição $(1,1)$ é igual a 1 e todas as outras são iguais a zero. Então, 


$$
\left\langle E_{11}\right\rangle=\left\{\left[\begin{array}{cccc}
a_{1} & 0 & \cdots & 0 \\
a_{2} & 0 & \cdots & 0 \\
\vdots & \vdots & \ddots & \vdots \\
a_{n} & 0 & \cdots & 0
\end{array}\right] ; a_{i} \in \mathbb{F}_{q}, 1 \leq i \leq n\right\} .
$$

Observa-se que um elemento não nulo em $\left\langle E_{11}\right\rangle$ é um idempotente se, e somente se, $a_{1}=1$. Então o número de idempotentes em $\left\langle E_{11}\right\rangle$ é $q^{n-1}$ e cada um deles é gerador de um ideal.

Seja $J=\langle M\rangle$, um ideal minimal à esquerda de $M_{n}\left(\mathbb{F}_{q}\right)$. Como posto $(M)=1$, existe uma matriz invertível $U \in M_{n}\left(\mathbb{F}_{q}\right)$, tal que, $U^{-1} M U=E_{11}$. A conjugação por $U$ induz um automorfismo

$$
\psi_{U}: M_{n}\left(\mathbb{F}_{q}\right) \rightarrow M_{n}\left(\mathbb{F}_{q}\right)
$$

tal que, $\psi_{U}(J)=\left\langle E_{11}\right\rangle$. Consequentemente, $J$ também tem $q^{n-1}$ geradores que são idempotentes.

Estenderemos este resultado para ideais à esquerda de posto $k, 1 \leq k \leq n$.

Seja $E(n, k)$ o conjunto de todas as matrizes $A=\left(a_{i j}\right)$ que possuem $k$ linhas, cujas posições serão denotadas por $i_{1}, i_{2}, \cdots, i_{k}$ tais que:

(i) toda linha de $A$, exceto estas, são linhas nulas;

(ii) $a_{i_{j} i_{j}}=1$ e $a_{i_{j}, h}=0$ se $h<i_{j}, 1 \leq j \leq k$;

(iii) $a_{i_{j}, h}=0$ para $h=i_{r}, j+1 \leq r \leq k$.

O conjunto dos números $i_{1}, i_{2}, \cdots, i_{k}$ chamaremos de posição pivô de $A$.

Claramente, toda matriz $E(n, k)$ é idempotente, de posto $k$.

Primeiramente afirmamos que cada matriz $E(n, k)$ gera um ideal diferente em $M_{n}\left(\mathbb{F}_{q}\right)$. De fato, dados $R, S \in E(n, k)$, se eles são diferentes em alguma posição pivô então, $W(R) \neq W(S)$. Portanto, $\langle R\rangle \neq\langle S\rangle$.

Por outro lado, se $R$ e $S$ têm a mesma posição pivô:

- Pela parte (ii) da Observação 3.7 se $[R]=[S]$ então, $R S=S$ e $S R=R$;

- Pela parte (ii) do Teorema 3.5 se $\langle R\rangle=\langle S\rangle$ então, $R S=R$ e $S R=S$;

mostrando assim que $R=S$.

Com isto, o número de elementos de $E(n, k)$ é igual ao número de ideais à esquerda de posto $k$ de $M_{n}\left(\mathbb{F}_{q}\right)$.

Exemplo 3.10. Nas condições acima, vamos descrever as matrizes $E(5,3)$, isto é, as matrizes idempotentes de posto 3 em $M_{5}\left(\mathbb{F}_{q}\right)$ e computaremos a quantidade delas explicitamente:

$$
\begin{aligned}
& {\left[\begin{array}{ccccc}
1 & 0 & 0 & a_{14} & a_{15} \\
0 & 1 & 0 & a_{24} & a_{25} \\
0 & 0 & 1 & a_{34} & a_{35} \\
0 & 0 & 0 & 0 & 0 \\
0 & 0 & 0 & 0 & 0
\end{array}\right],\left[\begin{array}{ccccc}
1 & 0 & a_{13} & 0 & a_{15} \\
0 & 1 & a_{23} & 0 & a_{25} \\
0 & 0 & 0 & 0 & 0 \\
0 & 0 & 0 & 1 & a_{45} \\
0 & 0 & 0 & 0 & 0
\end{array}\right],\left[\begin{array}{ccccc}
1 & 0 & a_{13} & a_{14} & 0 \\
0 & 1 & a_{23} & a_{24} & 0 \\
0 & 0 & 0 & 0 & 0 \\
0 & 0 & 0 & 0 & 0 \\
0 & 0 & 0 & 0 & 1
\end{array}\right],} \\
& {\left[\begin{array}{ccccc}
1 & a_{12} & 0 & 0 & a_{15} \\
0 & 0 & 0 & 0 & 0 \\
0 & 0 & 1 & 0 & a_{35} \\
0 & 0 & 0 & 1 & a_{45} \\
0 & 0 & 0 & 0 & 0
\end{array}\right],\left[\begin{array}{ccccc}
1 & a_{12} & 0 & a_{14} & 0 \\
0 & 0 & 0 & 0 & 0 \\
0 & 0 & 1 & a_{34} & 0 \\
0 & 0 & 0 & 0 & 0 \\
0 & 0 & 0 & 0 & 1
\end{array}\right],\left[\begin{array}{ccccc}
1 & a_{12} & a_{13} & 0 & 0 \\
0 & 0 & 0 & 0 & 0 \\
0 & 0 & 0 & 0 & 0 \\
0 & 0 & 0 & 1 & 0 \\
0 & 0 & 0 & 0 & 1
\end{array}\right],} \\
& {\left[\begin{array}{ccccc}
0 & 0 & 0 & 0 & 0 \\
0 & 1 & 0 & 0 & a_{25} \\
0 & 0 & 1 & 0 & a_{35} \\
0 & 0 & 0 & 1 & a_{45} \\
0 & 0 & 0 & 0 & 0
\end{array}\right],\left[\begin{array}{ccccc}
0 & 0 & 0 & 0 & 0 \\
0 & 1 & 0 & a_{24} & 0 \\
0 & 0 & 1 & a_{34} & 0 \\
0 & 0 & 0 & 0 & 0 \\
0 & 0 & 0 & 0 & 1
\end{array}\right],\left[\begin{array}{ccccc}
0 & 0 & 0 & 0 & 0 \\
0 & 1 & a_{23} & 0 & 0 \\
0 & 0 & 0 & 0 & 0 \\
0 & 0 & 0 & 1 & 0 \\
0 & 0 & 0 & 0 & 1
\end{array}\right] \text { e }\left[\begin{array}{ccccc}
0 & 0 & 0 & 0 & 0 \\
0 & 0 & 0 & 0 & 0 \\
0 & 0 & 1 & 0 & 0 \\
0 & 0 & 0 & 1 & 0 \\
0 & 0 & 0 & 0 & 1
\end{array}\right]}
\end{aligned}
$$


e cada $a_{i j} \in \mathbb{F}_{q}$.

Para computar o número de matrizes idempotentes diferentes, conforme as posições pivô das matrizes acima, contemos o número de variáveis livres, como são matrizes sobre $\mathbb{F}_{q}$ tal número é:

$$
S(5,3)=q^{6}+q^{5}+2 q^{4}+2 q^{3}+2 q^{2}+q+1=\frac{\left(q^{5}-1\right)\left(q^{4}-1\right)\left(q^{3}-1\right)}{\left(q^{3}-1\right)\left(q^{2}-1\right)(q-1)} .
$$

Lema 3.11. Com as notações acima, temos que

$$
S(n, k)=\frac{\left(q^{n}-1\right)\left(q^{n-1}-1\right)\left(q^{n-2}-1\right) \cdots\left(q^{n-k+1}-1\right)}{\left(q^{k}-1\right)\left(q^{k-1}-1\right)\left(q^{k-2}-1\right) \cdots(q-1)} .
$$

\section{Demonstração:}

A prova será feita por indução sobre $n$.

Se $n=1$ a igualdade se verifica diretamente.

Assumamos que o resultado seja válido para para $n-1$ e $k \leq n-1$.

Vamos computar separadamente o número de elementos de $E(n, k)$ que tem $n$ na posição pivô e aqueles que não o têm. Claramente, existem $S(n-1, k-1)$ idempotentes que têm entrada igual a 1 na posição $(n, n)$. Se a matriz tem entrada igual a zero na posição $(n, n)$, temos $k$ entradas em $\mathbb{F}_{q}$ na última coluna. Assim, existem $S(n-1, k) q^{k}$ de tais matrizes.

Logo, $S(n, k)=S(n-1, k-1)+S(n-1, k) q^{k}$.

Usando a hipótese de indução, obtemos:

$$
\begin{aligned}
S(n, k)= & \frac{\left(q^{n-1}-1\right) \cdots\left(q^{n-k+1}-1\right)}{\left(q^{k}-1\right) \cdots(q-1)}+ \\
& +\frac{\left(q^{n-1}-1\right) \cdots\left(q^{n-k}-1\right)}{\left(q^{k}-1\right) \cdots(q-1)} \cdot q^{k}= \\
= & \frac{\left(q^{n-1}-1\right) \cdots\left(q^{n-k+1}-1\right)}{\left(q^{k-1}-1\right) \cdots(q-1)}\left[\frac{q^{n-k}-1}{q^{k}-1}+q^{n-k}\right] \\
= & \frac{\left(q^{n-1}-1\right) \cdots\left(q^{n-k+1}-1\right)}{\left(q^{k-1}-1\right) \cdots(q-1)}\left[1+\frac{\left(q^{n-k}-1\right) q^{k}}{q^{k}-1}\right] \\
= & \frac{\left(q^{n-1}-1\right) \cdots\left(q^{n-k+1}-1\right)}{\left(q^{k-1}-1\right) \cdots(q-1)} \cdot \frac{\left(q^{n}-1\right)}{\left(q^{k}-1\right)} .
\end{aligned}
$$

Note que, o número de elementos em $E(n, k)$ encontrados acima é igual ao número de ideais à esquerda de posto $k$ em $M_{n}\left(\mathbb{F}_{q}\right)$, como vimos no Teorema 3.4.

Teorema 3.12. Os elementos do conjunto $E(n, k)$ são idempotentes geradores de diferentes ideais à esquerda de posto $k$ em $M_{n}\left(\mathbb{F}_{q}\right)$. Além disso, cada ideal à esquerda de posto $k$ tem $q^{(n-k) k}$ geradores idempotentes diferentes.

\section{Demonstração:}

Vamos começar provando a última afirmação do enunciado.

Seja $I_{k}$ a matriz identidade $k \times k$, e denotemos

$$
F_{k k}=\left[\begin{array}{cc}
I_{k} & 0 \\
0 & 0
\end{array}\right] \in M_{n}\left(\mathbb{F}_{q}\right) .
$$


Pelo Teorema 3.6, para toda $M=\left[\begin{array}{cc}A & C \\ B & D\end{array}\right] \in M_{n}\left(\mathbb{F}_{q}\right)$ e o idempotente $F_{k k}$, temos que todo outro idempotente gerador deste ideal é da forma:

$$
M_{0}=\left[\begin{array}{cc}
I_{k} & 0 \\
0 & 0
\end{array}\right]+\left[\begin{array}{cc}
0 & 0 \\
0 & I_{n-k}
\end{array}\right]\left[\begin{array}{cc}
A & C \\
B & D
\end{array}\right]\left[\begin{array}{cc}
I_{k} & 0 \\
0 & 0
\end{array}\right]=\left[\begin{array}{cc}
I_{k} & 0 \\
B & 0
\end{array}\right] .
$$

Consequentemente, este ideal tem $q^{(n-k) k}$ geradores idempotentes diferentes.

Com um argumento similar ao da demonstração do Teorema 3.9, mostra-se que todo ideal à esquerda de posto $k$ tem o mesmo número de idempotentes geradores que este ideal.

Perceba que as matrizes de posto $k$ possuem $k$ linhas linearmente independentes. O número total de escolhas de vetores em $\mathbb{F}_{q}^{n}$, que são linearmente independentes é:

$$
\left(q^{n}-1\right)\left(q^{n}-q\right)\left(q^{n}-q^{2}\right) \cdots\left(q^{n}-q^{k-1}\right) .
$$

Todos os ideais à esquerda de posto $k$ contém o mesmo número de matrizes deste posto, desde que eles sejam conjugados a $\left\langle\mathbb{F}_{k k}\right\rangle$, a conjugação leva matrizes de um dado posto em matrizes do mesmo posto. Como toda matriz de posto $k$ pertence a um e somente um dos ideais, pelo Teorema 3.12 acima, podemos concluir que é válido:

Teorema 3.13. Seja $k$ um inteiro tal que $1 \leqslant k \leqslant n$. Então, o número de matrizes de posto $k$ em $M_{n}\left(\mathbb{F}_{q}\right)$ é:

$$
q^{\frac{(k-1) k}{2}} \cdot \frac{\left(\left(q^{n}-1\right)\left(q^{n-1}-1\right)\left(q^{n-2}-1\right) \cdots\left(q^{n-k+1}-1\right)\right)^{2}}{\left(q^{k}-1\right)\left(q^{k-1}-1\right)\left(q^{k-2}-1\right) \cdots(q-1)} .
$$




\section{Capítulo 4}

\section{Grupos dos tipos $\mathscr{D}_{1}$ e $\mathscr{D}_{2}$}

Como já foi visto anteriormente, os grupos indecomponíveis finitos que estamos considerando são de um dos seguintes tipos:

\begin{tabular}{|l|l|c|}
\hline Classe & \multicolumn{1}{|c|}{ Geradores } & \multicolumn{1}{|c|}{ Grupos } \\
\hline $\mathscr{D}_{1}$ & $x, y, t_{1}$ & $\left\langle x, y, t_{1} ; x^{p}=y^{p}=t_{1}^{p^{m_{1}}}=1\right\rangle, m_{1} \geq 1$ \\
\hline $\mathscr{D}_{2}$ & $x, y, t_{1}$ & $\left\langle x, y, t_{1} ; x^{p}=y^{p}=t_{1}, t_{1}^{p^{m}}=1\right\rangle, m_{1} \geq 1$ \\
\hline $\mathscr{D}_{3}$ & $x, y, t_{1}, t_{2}$ & $\left\langle x, y, t_{1}, t_{2} ; x^{p}=t_{1}^{p^{m}}=t_{2}^{p^{m}}=1, y^{p}=t_{2}\right\rangle, m_{1}, m_{2} \geq 1$ \\
\hline $\mathscr{D}_{4}$ & $x, y, t_{1}, t_{2}$ & $\left\langle x, y, t_{1}, t_{2} ; x^{p}=t_{1}, y^{p}=t_{2}, t_{1}^{p^{m}}=t_{2}^{p^{m}}=1\right\rangle, m_{1}, m_{2} \geq 1$ \\
\hline $\mathscr{D}_{5}$ & $x, y, t_{1}, t_{2}, t_{3}$ & $\left\langle x, y, t_{1}, t_{2}, t_{3} ; x^{p}=t_{2}, y^{p}=t_{3}, t_{1}^{p^{m_{1}}}=t_{2}^{p^{m}}=t_{3}^{p^{m}}=1\right\rangle, m_{1}, m_{2}, m_{3} \geq 1$ \\
\hline
\end{tabular}

No que segue, explicitaremos alguns resultados que serão usados no decorrer deste capítulo.

Lema 4.1. Seja $x, y$ elementos de um dos grupos da tabela acima. Tem-se que:

$$
(x y)^{n}=x^{n} y^{n} s^{\frac{(n-1) n}{2}} \text {, para todo inteiro positivo } n \text {. }
$$

\section{Demonstração:}

A prova será por indução em $n$.

- Se $n=2$, temos $(x y)^{2}=x(y x) y=x^{2} y^{2} s$;

- Suponhamos que a igualdade seja válida para um inteiro $n$ positivo fixado e vamos provar que vale para $n+1$. Assim,

$$
(x y)^{n+1}=x\left(y x^{n}\right) y^{n} s^{\frac{(n-1) n}{2}}=x^{n+1} y^{n+1} s^{n} s^{\frac{(n-1) n}{2}}=x^{n+1} y^{n+1} s^{\frac{n(n+1)}{2}} .
$$

Vamos exibir uma nova presentação para os grupos do tipo $\mathscr{D}_{2}$, quando $p \neq 2$. Para o caso $p=2$ mantém-se a presentação original.

Dado um grupo $G_{2}$ um grupo do tipo $\mathscr{D}_{2}$, vamos considerar a mudança de variável: $y^{\prime}=x y^{-1}$. Então pelo 4.1, note que

$$
\left(y^{\prime}\right)^{p}=\left(x y^{-1}\right)^{p}=x^{p} y^{-p} s^{\frac{(p-1) p}{2}}=t_{1} t_{1}^{-1}\left(s^{p}\right)^{\frac{p-1}{2}}=1 \text {, pois } p \text { é ímpar. }
$$

Portanto, $\left(x y^{-1}\right)^{p}=1$.

Falta mostrar que $x y^{\prime}=y^{\prime} x s$. De fato,

$$
\begin{aligned}
{\left[x, y^{\prime}\right] } & =x y^{\prime} x^{-1} y^{-1}=x\left(x y^{-1}\right) x^{-1}\left(x y^{-1}\right)^{-1} \\
& =x^{2} y^{-1} x^{-1} y x^{-1}=x^{2} x^{-1} y^{-1} y x^{-1} s \\
& =s
\end{aligned}
$$


Assim, $G_{2}=\left\langle x, y^{\prime}, t_{1} ; x^{p}=t_{1}, t_{1}^{p^{m_{1}}}=\left(y^{\prime}\right)^{p}=1\right\rangle$.

Lema 4.2. Seja $G$ um grupo do tipo $\mathscr{D}_{1}$ ou $\mathscr{D}_{2}$, com $\mathscr{Z}(G)=\left\langle t_{1}\right\rangle \cong C_{p^{m_{1}}}$. Então, o número de componentes simples da parte não comutativa de $\mathbb{F} G$ é

$$
N=\frac{p^{m_{1}-1}(p-1)}{l},
$$

onde l é o tamanho das $q$-classes de elementos de ordem $p^{m_{1}}$, com $m_{1} \geq 1$.

\section{Demonstração:}

Lembramos que, pelo Teorema 2.16, o número de componentes simples da parte não comutativa de $\mathbb{F} G$ é igual ao número de $q$-classes de $\mathscr{Z}(G)$ menos o número de $q$-classes de $\mathscr{Z}(G) / G^{\prime}$.

Sabemos que $|\mathscr{Z}(G)|=p^{m_{1}}$ e $\left|\mathscr{Z}(G) / G^{\prime}\right|=p^{m_{1}-1}$, com $m_{1} \geq 1$, são grupos cíclicos, e o número de elementos de ordem $p^{r}$, com $0 \leq r \leq m_{1}-1$ é igual em ambos os grupos. Como a ordem da $q$ classe de um elemento depende apenas da ordem desse elemento, a diferença que queremos calcular é igual ao número de $q$-classes dos elementos de ordem $p^{m_{1}}$ em $\mathscr{Z}(G)$.

Seja $g \in \mathscr{Z}(G)$ um elemento de ordem $p^{m_{1}}$. Sua $q$-classe é:

$$
\left\{g, g^{q}, g^{q^{2}}, \cdots, g^{q^{l-1}}\right\}
$$

onde $l$ é o menor inteiro positivo tal que $q^{l} \equiv 1\left(\bmod p^{m_{1}}\right)$.

O número de elementos de ordem $p^{m_{1}}$ em $\mathscr{Z}(G)$ é $\varphi\left(p^{m_{1}}\right)=p^{m_{1}-1}(p-1)$. Logo, o número de componentes simples da parte não comutativa de $\mathbb{F} G$ é

$$
N=\frac{p^{m_{1}-1}(p-1)}{l}
$$

Teorema 4.3. Seja $G$ um grupo do tipo $\mathscr{D}_{1}$ ou $\mathscr{D}_{2}$, com $\mathscr{Z}(G)=\left\langle t_{1}\right\rangle \cong C_{p^{m_{1}}}$. Cada componente simples da parte não comutativa da álgebra $\mathbb{F} G$ é isomorfa a $M_{p}\left(\mathbb{F}_{q}(\alpha)\right)$ onde $\alpha$ é uma raiz primitiva $p^{m_{1}}$-ésima da unidade.

\section{Demonstração:}

Relembremos que podemos decompor $\mathbb{F} \mathscr{Z}(G)=\mathbb{F} \mathscr{Z}(G) e_{G^{\prime}} \oplus \mathbb{F} \mathscr{Z}(G)\left(1-e_{G^{\prime}}\right)$. Agora, como $\mathbb{F} \mathscr{Z}(G) e_{G^{\prime}} \cong \mathbb{F}\left(\mathscr{Z}(G) / G^{\prime}\right)$ as dimensões das componentes simples de $\mathbb{F} \mathscr{Z}(G) e_{G^{\prime}}$ devem corresponder aos tamanhos de $q$-classes de elementos de ordem $p^{r}$, com $0 \leq r \leq m_{1}-1$. Logo, as dimensões das componentes simples da parte não comutativa de $\mathbb{F} G$ correspondem à ordem das $q$-classes de elementos de ordem $p^{m_{1}}$. Consideremos $l$ o tamanho das $q$-classes de elementos de ordem $p^{m_{1}}$, com $m_{1} \geq 1$.

Como $\mathscr{Z}\left(M_{p}\left(\mathbb{F}_{i}\right)\right) \cong \mathbb{F}_{i}$ segue que $\left[\mathbb{F}_{i}: \mathbb{F}\right]=l$, donde $\left|\mathbb{F}_{i}\right|=q^{l}$ e $\left|\mathbb{F}_{i}^{*}\right|=q^{l}-1$.

Uma vez que $p^{m_{1}} \mid\left(q^{l}-1\right)$ e $\mathbb{F}_{q^{l}}^{*}$ munido com o produto é um grupo cíclico, ele possui um elemento $\alpha$ de ordem $p^{m_{1}}$, que é uma raiz primitiva $p^{m_{1}}$-ésima da unidade.

Então, $\mathbb{F}_{q}(\alpha) \subset \mathbb{F}_{q^{l}}$ e $\left[\mathbb{F}_{q}(\alpha): \mathbb{F}_{q}\right]=l^{\prime} \leq l$. Assim, $\mathbb{F}_{q}(\alpha) \cong \mathbb{F}_{q^{l^{\prime}}}$ e $o(\alpha) \mid\left(q^{l^{\prime}}-1\right)$, mas $p^{m_{1}} \mid\left(q^{l}-1\right)$ e $l$ é o menor inteiro positivo tal que isto acontece.

Portanto, $l^{\prime}=l$ e $\mathbb{F}_{q}(\alpha)=\mathbb{F}_{q^{l}}$. 
Nas próximas duas seções, explicitaremos o isomorfismo enunciado no Teorema 4.3, para $\mathscr{D}_{1}$ e $\mathscr{D}_{2}$.

\subsection{Correspondência na Álgebra das Matrizes para $\mathscr{D}_{1}$}

Nesta seção, consideramos um grupo do tipo $\mathscr{D}_{1}$ :

$$
G=\left\langle x, y, t_{1} ; x^{p}=y^{p}=t_{1}^{p^{m_{1}}}=1, m_{1} \geq 1, s=t_{1}^{p^{m_{1}-1}}, x y=y x s\right\rangle .
$$

Dado um idempotente central primitivo $e$ da parte não comutativa de $\mathbb{F} G$, sabemos que

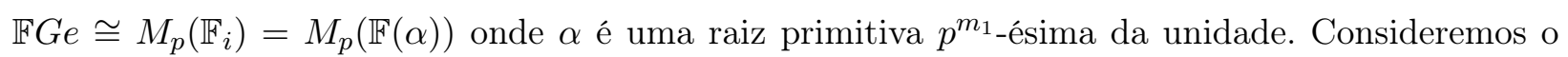
grupo $G l(p, \mathbb{F}(\alpha))$ das matrizes invertíveis de ordem $p$ sobre $\mathbb{F}(\alpha)$. Vamos descrever explicitamente um isomorfismo, este não é único.

Seja

$$
\varphi: G \rightarrow G l(p, \mathbb{F}(\alpha))
$$

definida por:

$$
\begin{aligned}
1_{G} \mapsto\left[\begin{array}{cccc}
1 & 0 & \cdots & 0 \\
0 & 1 & \cdots & 0 \\
\vdots & \vdots & \ddots & \vdots \\
0 & 0 & \cdots & 1
\end{array}\right] & \\
x \mapsto\left[\begin{array}{cccc}
1 & 0 & \cdots & 0 \\
0 & \alpha^{p^{m_{1}-1}} & \cdots & 0 \\
\vdots & \vdots & \ddots & \vdots \\
0 & 0 & \cdots & \alpha^{(p-1) p^{m_{1}-1}}
\end{array}\right] & y \mapsto\left[\begin{array}{ccccc}
0 & 0 & \cdots & 0 & 1 \\
1 & 0 & \cdots & 0 & 0 \\
0 & 1 & \cdots & 0 & 0 \\
\vdots & \vdots & \ddots & \vdots & \vdots \\
0 & 0 & \cdots & 1 & 0
\end{array}\right] \\
s \mapsto\left[\begin{array}{cccc}
\alpha^{p^{m_{1}-1}} & 0 & \ldots & 0 \\
0 & \alpha^{p^{m_{1}-1}} & \cdots & 0 \\
\vdots & \vdots & \ddots & \vdots \\
0 & 0 & \cdots & \alpha^{p^{m_{1}-1}}
\end{array}\right] & t_{1} \mapsto\left[\begin{array}{cccc}
\alpha & 0 & \cdots & 0 \\
0 & \alpha & \cdots & 0 \\
\vdots & \vdots & \ddots & \vdots \\
0 & 0 & \cdots & \alpha
\end{array}\right]
\end{aligned}
$$

$$
\begin{aligned}
& x y \mapsto\left[\begin{array}{ccccc}
0 & 0 & \cdots & 0 & 1 \\
\alpha^{p^{m_{1}-1}} & 0 & \cdots & 0 & 0 \\
0 & \alpha^{2 p^{m_{1}-1}} & \cdots & 0 & 0 \\
\vdots & \vdots & \ddots & \vdots & \vdots \\
0 & 0 & \cdots & \alpha^{(p-1) p^{m_{1}-1}} & 0
\end{array}\right] \\
& y x \mapsto\left[\begin{array}{ccccc}
0 & 0 & \cdots & 0 & \alpha^{(p-1) p^{m_{1}-1}} \\
1 & 0 & \cdots & 0 & 0 \\
0 & \alpha^{2 p^{m_{1}-1}} & \ldots & 0 & 0 \\
\vdots & \vdots & \ddots & \vdots & \vdots \\
0 & 0 & \cdots & \alpha^{(p-2) p^{m_{1}-1}} & 0
\end{array}\right] \text {. }
\end{aligned}
$$

Note que $\varphi$ possui as seguintes propriedades:

- o elemento $1_{G}$, identidade do grupo $G$ é levado na matriz identidade de ordem $p$;

- as respectivas matrizes associadas a $x, y, s$ elevadas à potência $p$ resultam na identidade;

- a relação $x y=y x s$, é verificada pelas respectivas imagens.

$\varphi$ é um homomorfismo de grupos, que por linearidade se estende a

$$
\varphi: \mathbb{F} G \rightarrow M_{p}(\mathbb{F}(\alpha))
$$


E por restrição

$$
\varphi: \mathbb{F} G e \rightarrow M_{p}(\mathbb{F}(\alpha))
$$

dada por:

$$
\begin{aligned}
& e \mapsto\left[\begin{array}{cccc}
1 & 0 & \cdots & 0 \\
0 & 1 & \cdots & 0 \\
\vdots & \vdots & \ddots & \vdots \\
0 & 0 & \cdots & 1
\end{array}\right] \\
& x e \mapsto\left[\begin{array}{cccc}
1 & 0 & \cdots & 0 \\
0 & \alpha^{p^{m_{1}-1}} & \cdots & 0 \\
\vdots & \vdots & \ddots & \vdots \\
0 & 0 & \cdots & \alpha^{(p-1) p^{m_{1}-1}}
\end{array}\right] \quad y e \mapsto\left[\begin{array}{ccccc}
0 & 0 & \cdots & 0 & 1 \\
1 & 0 & \cdots & 0 & 0 \\
0 & 1 & \cdots & 0 & 0 \\
\vdots & \vdots & \ddots & \vdots & \vdots \\
0 & 0 & \cdots & 1 & 0
\end{array}\right] \\
& s e \mapsto\left[\begin{array}{cccc}
\alpha^{p^{m_{1}-1}} & 0 & \cdots & 0 \\
0 & \alpha^{p^{m_{1}-1}} & \cdots & 0 \\
\vdots & \vdots & \ddots & \vdots \\
0 & 0 & \cdots & \alpha^{p^{m_{1}-1}}
\end{array}\right] \quad t_{1} e \mapsto\left[\begin{array}{cccc}
\alpha & 0 & \cdots & 0 \\
0 & \alpha & \cdots & 0 \\
\vdots & \vdots & \ddots & \vdots \\
0 & 0 & \cdots & \alpha
\end{array}\right] \\
& x y e \mapsto\left[\begin{array}{ccccc}
0 & 0 & \cdots & 0 & 1 \\
\alpha^{p^{m_{1}-1}} & 0 & \cdots & 0 & 0 \\
0 & \alpha^{2 p^{m_{1}-1}} & \cdots & 0 & 0 \\
\vdots & \vdots & \ddots & \vdots & \vdots \\
0 & 0 & \cdots & \alpha^{(p-1) p^{m_{1}-1}} & 0
\end{array}\right] \\
& y x e \mapsto\left[\begin{array}{ccccc}
0 & 0 & \cdots & 0 & \alpha^{(p-1) p^{m_{1}-1}} \\
1 & 0 & \cdots & 0 & 0 \\
0 & \alpha^{2 p^{m_{1}-1}} & \cdots & 0 & 0 \\
\vdots & \vdots & \ddots & \vdots & \vdots \\
0 & 0 & \cdots & \alpha^{(p-2) p^{m_{1}-1}} & 0
\end{array}\right]
\end{aligned}
$$

Com as identificações dadas acima, pode-se determinar a imagem de cada elemento de $\mathbb{F} G e$ no anel da matrizes $M_{p}(\mathbb{F}(\alpha))$. Aqui só faremos a identificação explicita dos elementos que são potências de $x e$ :

$$
\begin{gathered}
x^{2} e \mapsto\left[\begin{array}{cccc}
1 & 0 & \cdots & 0 \\
0 & \alpha^{2 p^{m_{1}}-1} & \cdots & 0 \\
\vdots & \vdots & \ddots & \vdots \\
0 & 0 & \cdots & \alpha^{2(p-1) p^{m_{1}-1}}
\end{array}\right] \quad x^{3} e \mapsto\left[\begin{array}{ccccc}
1 & 0 & \cdots & 0 \\
0 & \alpha^{3 p^{m_{1}-1}} & \cdots & 0 \\
\vdots & \vdots & \ddots & \vdots \\
0 & 0 & \cdots & \alpha^{3(p-1) p^{m_{1}-1}}
\end{array}\right] \\
x^{p} e \mapsto\left[\begin{array}{ccccc}
1 & 0 & \cdots & 0 \\
0 & \alpha^{p p^{m_{1}-1}} & \cdots & 0 \\
\vdots & \vdots & \ddots & \vdots \\
0 & 0 & \cdots & \alpha^{p(p-1) p^{m_{1}-1}}
\end{array}\right]=\left[\begin{array}{cccc}
1 & 0 & \cdots & 0 \\
0 & 1 & \cdots & 0 \\
\vdots & \vdots & \ddots & \vdots \\
0 & 0 & \cdots & 1
\end{array}\right] \cdots
\end{gathered}
$$

Assim, a matriz elementar $E_{11}$ da base canônica de $M_{p}(\mathbb{F}(\alpha))$ corresponde ao seguinte elemento na álgebra $\mathbb{F} G e$ :

$$
\left[\begin{array}{cccc}
1 & 0 & \cdots & 0 \\
0 & 0 & \cdots & 0 \\
\vdots & \vdots & \ddots & \vdots \\
0 & 0 & \cdots & 0
\end{array}\right] \mapsto \frac{\left(1+x+x^{2}+\cdots+x^{p-1}\right)}{p} e
$$

Agora, lembrando que

$$
\mathbb{F}(\alpha)=\left\{a_{0}+a_{1} \alpha+\cdots+a_{l-1} \alpha^{p^{m_{1}}-1} ; a_{i} \in \mathbb{F}, 0 \leq i \leq p^{m_{1}}-1\right\},
$$

vamos explicitar todas as matrizes elementares $\alpha^{r} E_{i j}$, com $0 \leq r \leq p^{m_{1}}-1$ e $1 \leq i, j \leq p$ da base 
canônica de $M_{p}(\mathbb{F}(\alpha))$, com seus correspondentes elementos na álgebra $\mathbb{F} G e$, como segue:

$$
\alpha^{r} E_{i j} \mapsto y^{j-1} \frac{\left(1+x+x^{2}+\cdots+x^{p-1}\right) e}{p} y^{1-i} t_{1}^{r} .
$$

Como queríamos, o isomorfismo está explicitado.

\subsection{Correspondência na Álgebra das Matrizes para $\mathscr{D}_{2}$}

Nesta seção, consideramos $G$ um grupo do tipo $\mathscr{D}_{2}^{\prime}$ :

$$
G=\left\langle x, y^{\prime}, t_{1} ; x^{p}=t_{1}, t_{1}^{p^{m_{1}}}=\left(y^{\prime}\right)^{p}=1, m_{1} \geq 1, s=t_{1}^{p^{m_{1}-1}}, x y^{\prime} s=y^{\prime} x\right\rangle .
$$

Dado um idempotente central primitivo $e$ da parte não comutativa de $\mathbb{F} G$, sabemos que $\mathbb{F} G e \cong$ $M_{p}\left(\mathbb{F}_{i}\right)=M_{p}(\mathbb{F}(\alpha))$ onde $\alpha$ é uma raiz primitiva $p^{m_{1}}$-ésima da unidade. Consideremos o grupo $G l(p, \mathbb{F}(\alpha))$ das matrizes invertíveis de ordem $p$ sobre $\mathbb{F}(\alpha)$. Vamos descrever explicitamente um isomorfismo.

De maneira análoga ao feito na seção anterior, temos

$$
\varphi: \mathbb{F} G e \rightarrow M_{p}(\mathbb{F}(\alpha))
$$

explicitaremos o isomorfismo consideramos:

$$
\begin{aligned}
& e \mapsto\left[\begin{array}{cccc}
1 & 0 & \cdots & 0 \\
0 & 1 & \cdots & 0 \\
\vdots & \vdots & \ddots & \vdots \\
0 & 0 & \cdots & 1
\end{array}\right] \\
& x e \mapsto\left[\begin{array}{ccccc}
0 & 0 & \cdots & 0 & \alpha \\
1 & 0 & \cdots & 0 & 0 \\
0 & 1 & \cdots & 0 & 0 \\
\vdots & \vdots & \ddots & \vdots & \vdots \\
0 & 0 & \cdots & 1 & 0
\end{array}\right] \quad y^{\prime} e \mapsto\left[\begin{array}{cccc}
1 & 0 & \cdots & 0 \\
0 & \alpha^{p^{m_{1}-1}} & \cdots & 0 \\
\vdots & \vdots & \ddots & \vdots \\
0 & 0 & \cdots & \alpha^{(p-1) p^{m_{1}-1}}
\end{array}\right] \\
& x^{p} e=t_{1} e \mapsto\left[\begin{array}{cccc}
\alpha & 0 & \cdots & 0 \\
0 & \alpha & \cdots & 0 \\
\vdots & \vdots & \ddots & \vdots \\
0 & 0 & \cdots & \alpha
\end{array}\right] \quad t_{1}^{p^{m_{1}-1}} e=s e \mapsto\left[\begin{array}{cccc}
\alpha^{p^{m_{1}-1}} & 0 & \cdots & 0 \\
0 & \alpha^{p^{m_{1}-1}} & \cdots & 0 \\
\vdots & \vdots & \ddots & \vdots \\
0 & 0 & \cdots & \alpha^{p^{m_{1}-1}}
\end{array}\right] \\
& x y^{\prime} e \mapsto\left[\begin{array}{ccccc}
0 & 0 & \cdots & 0 & \alpha^{(p-1) p^{m_{1}-1}+1} \\
1 & 0 & \cdots & 0 & 0 \\
0 & \alpha^{p^{m_{1}-1}} & \cdots & 0 & 0 \\
\vdots & \vdots & \ddots & \vdots & \vdots \\
0 & 0 & \cdots & \alpha^{(p-2) p^{m_{1}-1}} & 0
\end{array}\right] \\
& y^{\prime} x e \mapsto\left[\begin{array}{ccccc}
0 & 0 & \cdots & 0 & \alpha \\
\alpha^{p^{m_{1}-1}} & 0 & \cdots & 0 & 0 \\
0 & \alpha^{2 p^{m_{1}-1}} & \cdots & 0 & 0 \\
\vdots & \vdots & \ddots & \vdots & \vdots \\
0 & 0 & \cdots & \alpha^{(p-1) p^{m_{1}-1}} & 0
\end{array}\right] \text {. }
\end{aligned}
$$

Verifica-se que:

- o elemento $e$, identidade da álgebra $\mathbb{F} G e$ é levado na matriz identidade de ordem $p$;

- a matriz associada a $x e$ elevada a potência $p$ resulta na matriz associada ao elemento $t_{1} e$; 
- $y^{\prime p} e$ na imagem, se associa à matriz identidade de ordem $p$;

- a relação $x y^{\prime} e s=y^{\prime} x e$, é verificada pelas respectivas imagens.

Pode-se determinar a imagem de cada elemento de $\mathbb{F} G e$ no anel da matrizes $M_{p}(\mathbb{F}(\alpha))$. Aqui, explicitaremos as potências do elemento $y^{\prime} e$ :

$$
\begin{aligned}
y^{\prime 2} e \mapsto\left[\begin{array}{cccc}
1 & 0 & \cdots & 0 \\
0 & \alpha^{2 p^{m_{1}-1}} & \cdots & 0 \\
\vdots & \vdots & \ddots & \vdots \\
0 & 0 & \cdots & \alpha^{2(p-1) p^{m_{1}-1}}
\end{array}\right] \quad y^{\prime 3} e \mapsto\left[\begin{array}{ccccc}
1 & 0 & \cdots & 0 \\
0 & \alpha^{3 p^{m_{1}-1}} & \cdots & 0 \\
\vdots & \vdots & \ddots & \vdots \\
0 & 0 & \cdots & \alpha^{3(p-1) p^{m_{1}-1}}
\end{array}\right] \\
y^{\prime p} e \mapsto\left[\begin{array}{ccccc}
1 & 0 & \cdots & 0 \\
0 & \alpha^{p p^{m_{1}-1}} & \cdots & 0 \\
\vdots & \vdots & \ddots & \vdots \\
0 & 0 & \cdots & \alpha^{p(p-1) p^{m_{1}-1}}
\end{array}\right]=\left[\begin{array}{cccc}
1 & 0 & \cdots & 0 \\
0 & 1 & \cdots & 0 \\
\vdots & \vdots & \ddots & \vdots \\
0 & 0 & \cdots & 1
\end{array}\right] \cdots
\end{aligned}
$$

Com esta identificação, a matriz elementar $E_{11}$ da base canônica de $M_{p}(\mathbb{F}(\alpha))$ corresponde ao seguinte elemento na álgebra $\mathbb{F} G e$ :

$$
\left[\begin{array}{cccc}
1 & 0 & \cdots & 0 \\
0 & 0 & \cdots & 0 \\
\vdots & \vdots & \ddots & \vdots \\
0 & 0 & \cdots & 0
\end{array}\right] \mapsto \frac{\left(1+y^{\prime}+y^{\prime 2}+\cdots+y^{\prime p-1}\right)}{p} e
$$

Agora, como

$$
\mathbb{F}(\alpha)=\left\{a_{0}+a_{1} \alpha+\cdots+a_{p^{m_{1}-1}} \alpha^{p^{m_{1}}-1} ; a_{i} \in \mathbb{F}, 0 \leq i \leq p^{m_{1}}-1\right\},
$$

vamos determinar todas as matrizes elementares $\alpha^{r} E_{i j}$, com $0 \leq r \leq l-1$ e $1 \leq i, j \leq p$ da base canônica de $M_{p}(\mathbb{F}(\alpha))$, com seus correspondentes elementos na álgebra $\mathbb{F} G e$, como segue:

- se $i \geq j$ temos:

$$
\alpha^{r} E_{i j} \mapsto x^{j-1} \frac{\left(1+y^{\prime}+y^{\prime 2}+\cdots+y^{\prime p-1}\right) e}{p} x^{1-i} t_{1}^{r} ;
$$

- se $i<j$ temos:

$$
\alpha^{r} E_{i j} \mapsto \frac{\left(1+y^{\prime}+y^{\prime 2}+\cdots+y^{\prime p-1}\right) e}{p} x^{1-i} t_{1}^{l-(r+1)} .
$$

Pela correspondência exposta acima, ambos os espaços têm a mesma dimensão, e o isomorfismo foi descrito.

Mostraremos na próxima seção que, sob algumas condições, os códigos de grupo do tipo $\mathscr{D}_{3}$ podem ser estudados a partir de códigos de grupo dos tipos $\mathscr{D}_{1}$ ou $\mathscr{D}_{2}$.

\subsection{Relações de $\mathscr{D}_{3}$ com $\mathscr{D}_{1}$ e $\mathscr{D}_{2}$}

Começaremos calculamos o número de componentes simples da parte não comutativa de $\mathbb{F} G$ para o caso específico em que $G$ é um grupo do tipo $\mathscr{D}_{3}$ e estão verificadas as hipóteses do Teorema 1.21, que enunciaremos novamente:

Sejam $p$ um primo impar e $A$ um p-grupo abeliano de expoente $p^{r}$. Então o conjunto de idem- 
potentes dado em ??, é um conjunto de idempotentes primitivos em $\mathbb{F} G$ se, e somente se, são verificadas as seguintes condições:

(i) $p^{r}=4$ e $q \equiv 3(\bmod 4)$;

(ii) $o(q)=\varphi\left(p^{n}\right)$ em $U\left(\mathbb{Z}_{p^{n}}\right)$, onde $\varphi$ é a função de Euler.

Nestas condições vale:

Proposição 4.4. Sejam A um grupo abeliano com $p^{n}$ elementos e $\mathbb{F}$ um corpo com $q$ elementos, onde $p$ e q são primos entre si. Se $g \in A$ e o $(g)=p^{k} \operatorname{com} o(\bar{q})=\varphi\left(p^{n}\right)$ em $U\left(\mathbb{Z}_{p^{n}}\right)$, então a $q$-classe de $g$ tem $\varphi\left(p^{k}\right)$ elementos.

\section{Demonstração:}

Começamos observando que $\langle g\rangle$ é um subgrupo $A$ e consequentemente $p^{k} \leq p^{n}$. A $q$-classe de $g$ é dada pelo seguinte conjunto:

$$
\left\{g, g^{q}, g^{q^{2}}, \cdots, g^{q^{r-1}}\right\}
$$

onde $r$ é o menor inteiro positivo tal que $q^{r} \equiv 1\left(\bmod p^{k}\right)$.

Vamos mostrar que $r=\varphi\left(p^{k}\right)$.

Consideremos o epimorfismo de anéis

$$
\begin{aligned}
f: \mathbb{Z}_{p^{n}} & \rightarrow \mathbb{Z}_{p^{k}} \\
\bar{a} & \mapsto \tilde{a},
\end{aligned}
$$

o qual, por restrição, nos fornece o seguinte homomorfismo de grupos:

$$
\begin{aligned}
\bar{f}: U\left(\mathbb{Z}_{p^{n}}\right) & \rightarrow U\left(\mathbb{Z}_{p^{k}}\right) \\
\bar{a} & \mapsto \tilde{a} .
\end{aligned}
$$

Agora, se $\tilde{a} \in U\left(\mathbb{Z}_{p^{k}}\right)$ então $m d c(a, p)=1$. Logo, $\bar{a} \in U\left(\mathbb{Z}_{p^{n}}\right)$. Com isto, $\bar{f}$ é um epimorfismo de grupos.

Por outro lado, a cardinalidade de $U\left(\mathbb{Z}_{p^{n}}\right)$ é $\varphi\left(p^{n}\right)$. Como $o(\bar{q})=\varphi\left(p^{n}\right)$ em $U\left(\mathbb{Z}_{p^{n}}\right)$, temos que $\langle\bar{q}\rangle=U\left(\mathbb{Z}_{p^{n}}\right)$.

Pelo fato de que $\bar{f}$ é um epimorfismo, $\tilde{q}=\bar{f}(\bar{q})$ gera $U\left(\mathbb{Z}_{p^{k}}\right)$. Portanto, o $(\tilde{q})$ em $U\left(\mathbb{Z}_{p^{k}}\right)$ é $\varphi\left(p^{k}\right)=\left|U\left(\mathbb{Z}_{p^{k}}\right)\right|$, donde concluímos que $\varphi\left(p^{k}\right)$ é o menor inteiro positivo tal que

$$
q^{\varphi\left(p^{k}\right)} \equiv 1\left(\bmod p^{k}\right)
$$

isto é, a $q$-classe de $g$ tem $\varphi\left(p^{k}\right)$ elementos.

Se $G$ é um grupo do tipo $\mathscr{D}_{3}$, então

$$
\mathscr{Z}(G)=\left\langle t_{1}\right\rangle \times\left\langle t_{2}\right\rangle \cong C_{p^{m_{1}}} \times C_{p^{m_{2}}}
$$

é um $p$-grupo abeliano. Nas condições da Proposição 4.4, como $\tilde{q}=\bar{f}(\bar{q})$ gera $U\left(\mathbb{Z}_{p^{k}}\right)$, se $o(g)=p^{k}$ a $q$-classe de $g \in \mathscr{Z}(G)$ é

$$
\mathscr{C}_{g}=\left\{g^{i} ; i \in U\left(\mathbb{Z}_{p^{k}}\right)\right\}
$$


A demonstração a seguir é baseada em [5, Lem.4.1]:

Teorema 4.5. Seja $A=C_{p^{m_{1}}} \times C_{p^{m_{2}}}$, onde $m_{1} \geq m_{2} \geq 1$. Então o número de $q$-classes de $A$ cujos elementos tem ordem $p^{k}$ é:

(i) 1 , se $k=0$

(ii) $p^{k-1}(p+1)$, se $1 \leq k \leq m_{2}$

(iii) $p^{m_{2}}$, se $m_{2}<k \leq m_{1}$

O número de $q$-classes de $A$ é $1+(p+1)\left(\frac{p^{m_{2}-1}}{p-1}\right)+\left(m_{1}-m_{2}\right) p^{m_{2}}$.

\section{Demonstração:}

(i) Visto que tem um elemento de ordem 1 em $A$, existe uma única $q$-classe de ordem 1.

(ii) Um elemento $(a, b) \in A$ satisfaz

$$
(a, b)^{p^{k}}=1 \text { se, e somente se, } a^{p^{k}}=b^{p^{k}}=1 \text {, com } 1 \leq k \leq m_{2} .
$$

Consideremos $C_{p^{k}} \times C_{p^{k}} \cong\langle\beta\rangle \times\langle\gamma\rangle \subset A$. Um elemento $(a, b) \in\langle\beta\rangle \times\langle\gamma\rangle$ tem ordem $p^{k}$ se, e somente se, $(a, b) \in\langle\beta\rangle \times\langle\gamma\rangle \backslash\left\langle\beta^{p}\right\rangle \times\left\langle\gamma^{p}\right\rangle$, onde cada conjunto tem $p^{2 k}$ e $p^{2(k-1)}$ elementos. Assim, o número de elementos de ordem $p^{k}$ neste subgrupo é $p^{2 k}-p^{2(k-1)}$. Sabemos que a $q$-classe de $(a, b)$ tem $\varphi\left(p^{k}\right)$ elementos. Então,

$$
\frac{p^{2 k}-p^{2(k-1)}}{\varphi\left(p^{k}\right)}=\frac{p^{2(k-1)}\left(p^{2}-1\right)}{p^{k-1}(p-1)}=p^{k-1}(p+1),
$$

é o número de $q$-classes cujos elementos tem ordem $p^{k}$ em $A$, para $1 \leq k \leq m_{2}$.

Segue que, o número total destas $q$-classes é:

$$
(p+1)+p(p+1)+\cdots+p^{m_{2}-1}(p+1)=(p+1)\left(1+p+\cdots+p^{m_{2}-1}\right)=(p+1)\left(\frac{p^{m_{2}}-1}{p-1}\right) .
$$

(iii) Em $C_{p^{m_{1}}}$ existem $\varphi\left(p^{k}\right)$ elementos de ordem $p^{k}$, com $m_{2}<k \leq m_{1}$, o que implica que em $C_{p^{m_{1}}} \times C_{p^{m_{2}}}$ existem $\varphi\left(p^{k}\right) p^{m_{2}}$ elementos de ordem $p^{k}$. Como cada $q$-classe tem $\varphi\left(p^{k}\right)$ elementos, o número de $q$-classes destes elementos é:

$$
\frac{\varphi\left(p^{k}\right) p^{m_{2}}}{\varphi\left(p^{k}\right)}=p^{m_{2}}
$$

para cada $k$, com $m_{2}<k \leq m_{1}$.

Então, o número total de $q$-classes deste tipo é: $\left(m_{1}-m_{2}\right) p^{m_{2}}$.

Portanto, o número de $q$-classes de $A$ é

$$
1+(p+1)\left(\frac{p^{m_{2}}-1}{p-1}\right)+\left(m_{1}-m_{2}\right) p^{m_{2}} .
$$

Corolário 4.6. Seja $A=C_{p^{m_{1}-1}} \times C_{p^{m_{2}}}$, onde $m_{1}-1 \geq m_{2} \geq 1$. Então o número de q-classes de A cujos elementos tem ordem $p^{k}$ é:

(i) 1 , se $k=0$

(ii) $p^{k-1}(p+1)$, se $1 \leq k \leq m_{2}$ 
(iii) $p^{m_{2}}$, se $m_{2}<k \leq m_{1}-1$

Temos, $1+(p+1)\left(\frac{p^{m_{2}-1}}{p-1}\right)+\left(m_{1}-m_{2}-1\right) p^{m_{2}}$ computando o número de todas as $q$-classes de A.

Conforme o Teorema 4.5, no caso em que $m_{2} \geq m_{1} \geq 1$ tem-se:

(i) $\quad 1$, se $k=0$;

(ii) $p^{k-1}(p-1)$, se $1 \leq k \leq m_{1}-1$;

(ii') $p^{m_{1}-1}(p-1)$, se $k=m_{1}$;

(iii) $p^{m_{1}}$, se $m_{1}<k \leq m_{2}$.

Temos, $1+(p+1)\left(\frac{p^{m_{1}}-1}{p-1}\right)+\left(m_{2}-m_{1}\right) p^{m_{1}}$ computando o número de todas as $q$-classes de $A$.

E pelo Corolário 4.6, no caso em que $m_{2} \geq m_{1} \geq 1$ tem-se:

(i) 1 , se $k=0$;

(ii) $p^{k-1}(p-1)$, se $1 \leq k \leq m_{1}-1$;

(iii) $p^{m_{1}-1}$, se $m_{1}-1<k \leq m_{2}$.

Temos, $1+(p+1)\left(\frac{p^{m_{1}}-1}{p-1}\right)+\left(m_{2}-m_{1}+1\right) p^{m_{1}-1}$ computando o número de todas as $q$-classes de $A$.

Lembramos que pela Proposição 2.16, o número de $q$-classes de $\mathscr{Z}(G)$ menos o número de $q$-classes de $\mathscr{Z}(G) / G^{\prime}$, é o número de $q$-classes da parte não comutativa de $\mathbb{F} G$.

Teorema 4.7. Seja $G$ um grupo do tipo $\mathscr{D}_{3}$ com $\mathscr{Z}(G)=C_{p^{m_{1}}} \times C_{p^{m_{2}}}$, então o número de q-classes da parte não comutativa de $\mathbb{F} G$ é:

(i) $p^{m_{2}}$, se $m_{1} \geq m_{2} \geq 1$;

(ii) $p^{m_{1}-1}\left(\left(m_{2}-m_{1}\right)(p-1)+p\right)$, se $m_{2} \geq m_{1} \geq 1$.

\section{Demonstração:}

- Se $m_{1} \geq m_{2} \geq 1$ :

$1+(p+1)\left(\frac{p^{m_{2}}-1}{p-1}\right)+\left(m_{1}-m_{2}\right) p^{m_{2}}-\left(1+(p+1)\left(\frac{p^{m_{2}}-1}{p-1}\right)+\left(m_{1}-m_{2}-1\right) p^{m_{2}}\right)$

$$
=p^{m_{2}}
$$

- Se $m_{2} \geq m_{1} \geq 1$ :

$1+(p+1)\left(\frac{p^{m_{1}}-1}{p-1}\right)+\left(m_{2}-m_{1}\right) p^{m_{1}}-\left(1+(p+1)\left(\frac{p^{m_{1}}-1}{p-1}\right)+\left(m_{2}-m_{1}+1\right) p^{m_{1}-1}\right)$

$$
=p^{m_{1}-1}\left(\left(m_{2}-m_{1}\right)(p-1)+p\right) \text {. }
$$

No que segue vamos explicitar o conjunto de idempotentes geradores da parte não comutativa de $\mathbb{F} G$, para $G$ um grupo do tipo $\mathscr{D}_{3}$ com

$$
\mathscr{Z}(G)=\left\langle t_{1}\right\rangle \times\left\langle t_{2}\right\rangle \cong C_{p^{m_{1}}} \times C_{p^{m_{2}}} \text { e } m_{1} \geq m_{2} \geq 1 .
$$


Lema 4.8. Sejam $\mathscr{Z}(G)$ nas condições anteriores e $a_{r}=t_{2} t_{1}^{r p^{m_{1}-m_{2}}} 0 \leq r \leq p^{m_{2}}-1$. São válidas as seguintes afirmações:

(i) $\left\langle a_{r}\right\rangle$ é cocíclico em $\mathscr{Z}(G)$. Além disso, $\left\langle a_{r}\right\rangle\langle s\rangle$ é o único subgrupo que contém $\left\langle a_{r}\right\rangle$ tal que $\left[\left\langle a_{r}\right\rangle\langle s\rangle:\left\langle a_{r}\right\rangle\right]=p$.

(ii) Os elementos da forma $\widehat{\left\langle a_{r}\right\rangle}\left(1-e_{G^{\prime}}\right) \in \mathbb{F} G\left(1-e_{G^{\prime}}\right)$ são idempotentes da parte não comutativa de $\mathbb{F} G$, distintos dois a dois e o número deles é $p^{m_{2}}$.

\section{Demonstração:}

(i) Afirmação: $\mathscr{Z}(G)=\left\langle t_{2} t_{1}^{r p^{m_{1}-m_{2}}}\right\rangle \times\left\langle t_{1}\right\rangle=\left\langle a_{r}\right\rangle \times\left\langle t_{1}\right\rangle$.

Primeiramente observemos que $o\left(a_{r}\right)=p^{m_{2}}, 0 \leq r \leq p^{m_{2}}-1$, e verificamos que $\left\langle a_{r}\right\rangle \cap\left\langle t_{1}\right\rangle=\{1\}$, pois se existirem inteiros $j, k, \operatorname{com} 0 \leq j, k \leq p^{m_{2}}-1$, tais que $a_{r}^{j}=t_{1}^{k}$ então,

$$
\left(t_{2} t_{1}^{r p^{m_{1}-m_{2}}}\right)^{j}=t_{1}^{k} \text { o que implica } j=0 \text { e } a_{r}=1 .
$$

Como ambos são subgrupos normais de $\mathscr{Z}(G)$, temos que

$$
\begin{gathered}
\mathscr{Z}(G)=\left\langle a_{r}\right\rangle \cdot\left\langle t_{1}\right\rangle, \text { onde } \\
t_{1} \in\left\langle a_{r}\right\rangle \cdot\left\langle t_{1}\right\rangle \mathrm{e} \\
t_{2}=\left(t_{2} t_{1}^{r p^{m_{1}-m_{2}}}\right) t_{1}^{-r p^{m_{1}-m_{2}}}=a_{r} t_{1}^{-r p^{m_{1}-m_{2}}}, 0 \leq r \leq p^{m_{2}}-1 .
\end{gathered}
$$

Então,

$$
\frac{\mathscr{Z}(G)}{\left\langle a_{r}\right\rangle} \cong\left\langle t_{1}\right\rangle \text { que é cíclico. }
$$

Portanto, $\left\langle a_{r}\right\rangle$ é cocíclico em $\mathscr{Z}(G)$.

De forma análoga, o único cocíclico de índice $p$ que contém $\left\langle a_{r}\right\rangle$ é $\left\langle a_{r}\right\rangle\langle s\rangle$.

(ii) Como $\widehat{G^{\prime}}=e_{G^{\prime}}=\widehat{s}$, temos que $\widehat{\left\langle a_{r}\right\rangle}-\widehat{\left\langle a_{r}\right\rangle\langle s\rangle}=\widehat{\left\langle a_{r}\right\rangle}\left(1-e_{G^{\prime}}\right)$ é idempotente da parte não comutativa de $\mathbb{F} G$, quando $G$ é um grupo do tipo $\mathscr{D}_{3}$, e os elementos desta forma são distintos dois a dois e um total de $p^{m_{2}}$ pois $0 \leq r \leq p^{m_{2}}-1$.

Teorema 4.9. Seja $G$ um grupo do tipo $\mathscr{D}_{3}$, nas condições do Lema 4.8.

Tem-se que, $\frac{G}{\left\langle a_{r}\right\rangle} \cong H$ :

(i) Caso $m_{1}>m_{2}$, então $H$ é um grupo do tipo $\mathscr{D}_{1}$

(ii) Caso $m_{1}=m_{2}$, então $H$ é um grupo do tipo $\mathscr{D}_{1}$, se $p \mid r$ e $H$ é um grupo do tipo $\mathscr{D}_{2}$, se $p \nmid r$.

\section{Demonstração:}

Se $G$ é um grupo do tipo $\mathscr{D}_{3}$, onde $m_{1} \geq m_{2}$, então $G$ tem as seguintes relações:

$$
x^{p}=t_{1}^{p^{m_{1}}}=t_{2}^{p^{m_{2}}}=1, y^{p}=t_{2} .
$$

Então, $G /\left\langle t_{2} t_{1}^{r p^{m_{1}-m_{2}}}\right\rangle, 0 \leq r \leq p^{m_{2}}-1$ resulta que

$$
\overline{t_{2} t_{1}^{r p^{m_{1}-m_{2}}}}=\overline{1} \text { o que implica } \overline{t_{2}}=\overline{t_{1}^{-r p^{m_{1}-m_{2}}}}, r p^{m_{1}-m_{2}}<p^{m_{1}} \text {. }
$$


(i) Se $m_{1}>m_{2}$ então $m_{1}-m_{2}=k>0$. Assim,

$$
y^{p}=t_{1}^{-r p^{m_{1}-m_{2}}}=t_{1}^{-r p^{k}} \text { o que implica } y^{\prime p}=\left(y t_{1}^{r p^{k-1}}\right)^{p}=1 .
$$

Portanto, como $t_{1} \in \mathscr{Z}(G)$, um grupo do tipo $\mathscr{D}_{1}$ pode ser reescrito da seguinte forma:

$$
\left\langle x, y^{\prime}, t_{1} ; x^{p}=y^{\prime p}=\left(y t_{1}^{-r p^{k-1}}\right)^{p}=t_{1}^{p^{m_{1}}}=1, m_{1} \geq 1 ; x y^{\prime}=y^{\prime} x s\right\rangle .
$$

Donde concluímos que, $H=\frac{G}{\left\langle a_{r}\right\rangle}$ um grupo do tipo $\mathscr{D}_{1}$, se $m_{1}>m_{2}$.

(ii) Se $m_{1}=m_{2}$,

- quando $p \mid r$

$$
y^{p}=t_{1}^{-r} \text { o que implica }\left(y^{\prime}\right)^{p}=\left(y t_{1}^{-\frac{r}{p}}\right)^{p}=y^{p} t_{1}^{-r}=1 .
$$

Donde concluímos que, existe $H$ um grupo do tipo $\mathscr{D}_{1}$ tal que, $\frac{G}{\left\langle a_{r}\right\rangle} \cong H$, se $m_{1}=m_{2}$ e $p \mid r$.

- quando $p \nmid r$

Considerando a mudança de variáveis: $x_{1}=y^{l}$ e $y_{1}=x^{t}$ temos que

$$
\begin{gathered}
\left(x_{1}\right)^{p}=\left(y^{l}\right)^{p}=\left(y^{p}\right)^{l}=\left(t_{1}^{-r}\right)^{l}=t_{1}^{-r l} \quad \mathrm{e} \\
\left(y_{1}\right)^{p}=\left(x^{p}\right)^{t}=1 .
\end{gathered}
$$

A mudança de variáveis deve verificar:

$$
x_{1} y_{1}=y_{1} x_{1} s .
$$

Assim,

$$
x_{1} y_{1}=y^{l} x^{t}=x^{t} y^{l} s^{t l}
$$

É necessário que $t l \equiv 1(\bmod (o(s))$, ou seja, $t l \equiv 1(\bmod p)$. Tomemos, $t=-r$.

Portanto,

$$
\begin{gathered}
\left(x_{1}\right)^{p}=\left(y^{l}\right)^{p}=\left(y^{p}\right)^{l}=\left(t_{1}^{-r}\right)^{l}=t_{1}^{-r l}=t_{1} \quad \mathrm{e} \\
\left(y_{1}\right)^{p}=x^{-r p}=1 .
\end{gathered}
$$

Donde concluímos que, existe $H=\frac{G}{\left\langle a_{r}\right\rangle}$ um grupo do tipo $\mathscr{D}_{2}^{\prime}$, que naturalmente é o tipo $\mathscr{D}_{2}$, se $m_{1}=m_{2}$ e $p \nmid r$.

Nas condições do Teorema acima,

$$
\mathbb{F} G \widehat{\left\langle a_{r}\right\rangle} \cong \mathbb{F}\left(\frac{G}{\left\langle a_{r}\right\rangle}\right)=\mathbb{F} H \cong \mathbb{F} H e_{H^{\prime}} \oplus \mathbb{F} H\left(1-e_{H^{\prime}}\right)
$$

Vamos explicitar um isomorfismo $\psi: \mathbb{F} G \widehat{\left\langle a_{r}\right\rangle} \rightarrow \mathbb{F} H$ 
Seja $T$ uma transversal de $\left\langle a_{r}\right\rangle$ em $G$, então todo $\alpha \in \mathbb{F} G \widehat{\left\langle a_{r}\right\rangle}$ se escreve de forma única como $\alpha=\sum_{t \in T} \alpha_{t} \widehat{\left\langle a_{r}\right\rangle} t$, com $\alpha_{t} \in \mathbb{F} G$.

Assim, $\psi\left(\sum_{t \in T} \alpha_{t} \widehat{\left\langle a_{r}\right\rangle} t\right)=\sum_{t \in T} \alpha_{t} \bar{t}$.

Ainda,

$$
\mathbb{F} G \widehat{\left\langle a_{r}\right\rangle} \cong \mathbb{F} G \widehat{\left\langle a_{r}\right\rangle} e_{G^{\prime}} \oplus \mathbb{F} G \widehat{\left\langle a_{r}\right\rangle}\left(1-e_{G^{\prime}}\right) .
$$

Portanto,

$$
\mathbb{F} G \widehat{\left\langle a_{r}\right\rangle}\left(1-e_{G^{\prime}}\right) \cong \mathbb{F} H\left(1-e_{H^{\prime}}\right)
$$

e é uma restrição de $\psi$.

Disto decorre que todo ideal $I$ de $\mathbb{F} G \widehat{\left\langle a_{r}\right\rangle}\left(1-e_{G^{\prime}}\right)$ é levado por $\psi$ em um ideal $\bar{I}$ de $\mathbb{F} H\left(1-e_{H^{\prime}}\right)$.

Donde concluímos que se o peso do elemento $\psi(\alpha)$ é $k_{\alpha}$, denotado por $\omega(\psi(\alpha))=k_{\alpha}$, então $\omega(\alpha)=p^{m_{2}} k_{\alpha}$, onde $p^{m_{2}}$ é a ordem do grupo $\left\langle a_{r}\right\rangle$.

Se $\bar{\theta}$ é a função distribuição de peso de $\bar{I}$, então a distribuição de peso de $I$ é dada por:

$$
\begin{gathered}
\theta(x)=\theta\left(\frac{x}{p^{m_{2}}}\right), \text { se } p^{m_{2}} \mid x \\
\theta(x)=0, \text { se } p^{m_{2}} \nmid x .
\end{gathered}
$$

Isto indica que, fazendo um estudo análogo para os grupos dos tipos $\mathscr{D}_{4}$ e $\mathscr{D}_{5}$ o resultado é similar.

No capítulo que segue, ilustramos com exemplos alguns dos resultados obtidos no Capítulo 2 bem como explicitaremos códigos em $\mathbb{F} G$ e seus parâmetros. 


\section{Capítulo 5}

\section{Exemplos}

Neste capítulo, explicitaremos a decomposição em componentes simples de duas álgebras de grupo e determinaremos dois códigos laterais com os mesmos parâmetros. Tais códigos têm parâmetros iguais aos melhores códigos conhecidos para o mesmo comprimento e dimensão, segundo o site www.codetables.de [10, Linear Codes]. Também explicitamos um código numa álgebra em que o gerador idempotente da parte não comutativa não é primitivo.

Exemplo 5.1. Seja $G$ um grupo do tipo $\mathscr{D}_{1}, \operatorname{com} p=3$ e $m_{1}=1$. Então,

$$
G=\left\langle x, y, t_{1} ; x^{3}=y^{3}=t_{1}^{3}=1, s=[x, y]=t_{1}\right\rangle .
$$

Vamos explicitar as componentes simples de $\mathbb{F}_{2} G$ e um código lateral com sua respectiva distribuição de peso.

Temos que $|G|=27$ e $G^{\prime}=\left\{1, s, s^{2}\right\}$. Vamos trabalhar sobre $\mathbb{F}_{2}$, que denotaremos simplesmente por $\mathbb{F}$.

O conjunto das 2-classes das classes de conjugação de $G$ é:

$$
\begin{aligned}
\{ & \{1\},\left\{\{s\},\left\{s^{2}\right\}\right\}, \\
& \left\{\left\{x, x s, x s^{2}\right\},\left\{x^{2}, x^{2} s, x^{2} s^{2}\right\}\right\}, \\
& \left\{\left\{y, y s, y s^{2}\right\},\left\{y^{2}, y^{2} s, y^{2} s^{2}\right\}\right\}, \\
& \left\{\left\{x y, x y s, x y s^{2}\right\},\left\{x^{2} y^{2}, x^{2} y^{2} s, x^{2} y^{2} s^{2}\right\}\right\}, \\
& \left.\left\{\left\{x^{2} y, x^{2} y s, x^{2} y s^{2}\right\},\left\{x y^{2}, x y^{2} s, x y^{2} s^{2}\right\}\right\} \quad\right\} .
\end{aligned}
$$

Pelo Teorema 1.18, segue que $\mathbb{F} G$ tem 6 componentes simples.

Agora analisemos a parte comutativa da álgebra $\mathbb{F} G$. Observemos que

$$
\frac{G}{G^{\prime}}=\left\{\overline{1}, \bar{x}, \overline{x^{2}}, \bar{y}, \overline{y^{2}}, \overline{x y}, \overline{x^{2} y}, \overline{x y^{2}}, \overline{x^{2} y^{2}}\right\}
$$

e o conjunto das respectivas 2-classes é:

$$
\begin{aligned}
& \left\{\{\overline{1}\}, \quad\left\{\{\bar{x}\},\left\{\overline{x^{2}}\right\}\right\}, \quad\left\{\{\bar{y}\},\left\{\overline{y^{2}}\right\}\right\},\right. \\
& \left.\left\{\{\overline{x y}\},\left\{\overline{x^{2} y^{2}}\right\}\right\}, \quad\left\{\left\{\overline{x^{2} y}\right\},\left\{\overline{x y^{2}}\right\}\right\}\right\} .
\end{aligned}
$$

Donde temos cinco 2-classes em $G / G^{\prime}$. Como cada 2-classe não trivial contém dois elementos, a dimensão da respectiva componente é dois sobre $\mathbb{F}$. 
Portanto, se $\xi$ é uma raíz 2-ésima primitiva da unidade, então

$$
\mathbb{F}\left(\frac{G}{G^{\prime}}\right) \cong \mathbb{F} \oplus \mathbb{F}(\xi) \oplus \mathbb{F}(\xi) \oplus \mathbb{F}(\xi) \oplus \mathbb{F}(\xi),
$$

é de dimensão 9 sobre $\mathbb{F}$.

Observação 5.2. $\mathbb{F} G$ possui $6-5=1$ componentes simples na parte não comutativa, cuja dimensão é $|G|-\left|G / G^{\prime}\right|=27-9=18$ sobre $\mathbb{F}$.

Como a componente não comutativa deve ser uma álgebra de matrizes de ordem $3 \times 3$, conclui-se que:

$$
\mathbb{F} G \cong \mathbb{F} \oplus \mathbb{F}(\xi) \oplus \mathbb{F}(\xi) \oplus \mathbb{F}(\xi) \oplus \mathbb{F}(\xi) \oplus M_{3}\left(\mathbb{F}_{4}\right)
$$

Como os códigos centrais de $\mathbb{F} G$ são combinatoriamente equivalentes a abelianos, temos interesse em trabalhar com códigos na parte não comutativa de $\mathbb{F} G$.

O idempotente gerador da parte não comutativa de $\mathbb{F} G$ é

$$
e=1-e_{G^{\prime}}=1-\frac{1+s+s^{2}}{3}=s+s^{2} .
$$

Portanto, $\mathbb{F} G e \cong M_{3}\left(\mathbb{F}_{4}\right)$ de dimensão 18 sobre $\mathbb{F}_{2}$.

Afirmamos que uma base da álgebra $\mathbb{F} G e$ é:

$$
\begin{gathered}
B=\left\{e, x e, x^{2} e, y e, y^{2} e, x y e, x^{2} y e, x y^{2} e, x^{2} y^{2} e,\right. \\
\left.e s, x e s, x^{2} e s, y e s, y^{2} e s, x y e s, x^{2} y e s, x y^{2} e s, x^{2} y^{2} e s\right\} .
\end{gathered}
$$

De fato, basta mostrar que estes elementos são linearmente independentes. Note que se

$$
\begin{gathered}
{\left[\alpha_{1}+\alpha_{2} x+\alpha_{3} x^{2}+\alpha_{4} y+\alpha_{5} y^{2}+\alpha_{6} x y+\alpha_{7} x^{2} y+\alpha_{8} x y^{2}+\alpha_{9} x^{2} y^{2}+\alpha_{10} s+\right.} \\
\left.\alpha_{11} x s+\alpha_{12} x^{2} s+\alpha_{13} y s+\alpha_{14} y^{2} s+\alpha_{15} x y s+\alpha_{16} x^{2} y s+\alpha_{17} x y^{2} s+\alpha_{18} x^{2} y^{2} s\right] e=0,
\end{gathered}
$$

onde $\alpha_{i} \in \mathbb{F}$ com $1 \leq i \leq 18$, reorganizando temos:

$$
\begin{gathered}
\left(\alpha_{1}+\alpha_{10} s\right) e+\left(\alpha_{2} x+\alpha_{11} x s\right) e+\left(\alpha_{3} x^{2}+\alpha_{12} x^{2} s\right) e+\left(\alpha_{4} y+\alpha_{13} y s\right) e+ \\
\left(\alpha_{5} y^{2}+\alpha_{14} y^{2} s\right) e+\left(\alpha_{6} x y+\alpha_{15} x y s\right) e+\left(\alpha_{7} x^{2} y+\alpha_{16} x^{2} y s\right) e+ \\
\left(\alpha_{8} x y^{2}+\alpha_{17} x y^{2} s\right) e+\left(\alpha_{9} x^{2} y^{2}+\alpha_{18} x^{2} y^{2} s\right) e=0 .
\end{gathered}
$$

As parcelas da soma acima têm suportes dois a dois disjuntos. Logo, para a soma ser nula, cada parcela deve ser igual a zero.

Recorde que: $e=s+s^{2}$ e $e s=1+s^{2}$. Assim,

$$
\alpha_{1} e+\alpha_{10} e s=0 \text { o que implica } \alpha_{10}+\alpha_{1} s+\left(\alpha_{1}+\alpha_{10}\right) s^{2}=0 \text { o que implica } \alpha_{1}=\alpha_{10}=0 \text {. }
$$

Nas parcelas restantes, o argumento é similar. Portanto, $B$ é uma base de $\mathbb{F} G e$

No que segue vamos explicitar um código desta álgebra

$$
\mathbb{F} G e \cong M_{3}\left(\mathbb{F}_{4}\right) \cong M_{3}(\mathbb{F}(\alpha))
$$

onde $\alpha$ é uma raiz cúbica primitiva da unidade que verifica $\alpha^{2}=1+\alpha$, conforme Teorema 4.3. 
Começaremos determinando a imagem de cada elemento da álgebra $\mathbb{F} G e$ com o seu correspondente elemento no anel das matrizes $M_{3}(\mathbb{F}(\alpha))$ :

$$
\begin{aligned}
& e \mapsto {\left[\begin{array}{ccc}
1 & 0 & 0 \\
0 & 1 & 0 \\
0 & 0 & 1
\end{array}\right] } \\
& x y e \mapsto\left[\begin{array}{ccc}
0 & 1 & 0 \\
0 & 0 & \alpha \\
\alpha^{2} & 0 & 0
\end{array}\right] \\
& x^{2} y e \mapsto\left[\begin{array}{ccc}
0 & 1 & 0 \\
0 & 0 & \alpha^{2} \\
\alpha & 0 & 0
\end{array}\right] \\
& \text { es } \mapsto\left[\begin{array}{ccc}
\alpha & 0 & 0 \\
0 & \alpha & 0 \\
0 & 0 & \alpha
\end{array}\right] \\
& \text { xyes } \mapsto\left[\begin{array}{ccc}
0 & \alpha & 0 \\
0 & 0 & \alpha^{2} \\
1 & 0 & 0
\end{array}\right] \\
& x^{2} \text { yes } \mapsto\left[\begin{array}{ccc}
0 & \alpha & 0 \\
0 & 0 & 1 \\
\alpha^{2} & 0 & 0
\end{array}\right]
\end{aligned}
$$$$
x e \mapsto\left[\begin{array}{lll}
1 & 0 & 0 \\
0 & \alpha & 0 \\
0 & 0 & \alpha^{2}
\end{array}\right]
$$$$
x^{2} e \mapsto\left[\begin{array}{ccc}
1 & 0 & 0 \\
0 & \alpha^{2} & 0 \\
0 & 0 & \alpha
\end{array}\right]
$$$$
x y^{2} e \mapsto\left[\begin{array}{ccc}
0 & 0 & 1 \\
\alpha & 0 & 0 \\
0 & \alpha^{2} & 0
\end{array}\right]
$$$$
x e s \mapsto\left[\begin{array}{ccc}
\alpha & 0 & 0 \\
0 & \alpha^{2} & 0 \\
0 & 0 & 1
\end{array}\right]
$$$$
x^{2} e s \mapsto\left[\begin{array}{ccc}
\alpha & 0 & 0 \\
0 & 1 & 0 \\
0 & 0 & \alpha^{2}
\end{array}\right]
$$$$
x y^{2} e s \mapsto\left[\begin{array}{ccc}
0 & 0 & \alpha \\
\alpha^{2} & 0 & 0 \\
0 & 1 & 0
\end{array}\right]
$$

$$
\begin{aligned}
& y e \mapsto\left[\begin{array}{lll}
0 & 1 & 0 \\
0 & 0 & 1 \\
1 & 0 & 0
\end{array}\right] \\
& y^{2} e \mapsto\left[\begin{array}{lll}
0 & 0 & 1 \\
1 & 0 & 0 \\
0 & 1 & 0
\end{array}\right] \\
& x^{2} y^{2} e \mapsto\left[\begin{array}{ccc}
0 & 0 & 1 \\
\alpha^{2} & 0 & 0 \\
0 & \alpha & 0
\end{array}\right] \\
& y e s \mapsto\left[\begin{array}{lll}
0 & \alpha & 0 \\
0 & 0 & \alpha \\
\alpha & 0 & 0
\end{array}\right] \\
& y^{2} e s \mapsto\left[\begin{array}{lll}
0 & 0 & \alpha \\
\alpha & 0 & 0 \\
0 & \alpha & 0
\end{array}\right] \\
& x^{2} y^{2} e s \mapsto\left[\begin{array}{lll}
0 & 0 & \alpha \\
1 & 0 & 0 \\
0 & \alpha^{2} & 0
\end{array}\right]
\end{aligned}
$$

Verifica-se que:

- o elemento $e$, identidade da álgebra FGe é levado na matriz identidade de ordem 3;

- as respectivas matrizes associadas a $x e, y e$, se elevadas á potência 3 resultam na identidade;

- finalmente, a relação xyes =yxe, é verificada pelas respectivas imagens.

Com a identificação dada acima, os elementos da base canônica do anel das matrizes $M_{3}\left(\mathbb{F}_{4}\right)$, correspondem aos seguintes elementos na álgebra $\mathbb{F} G e$ :

$$
\begin{aligned}
& {\left[\begin{array}{lll}
1 & 0 & 0 \\
0 & 0 & 0 \\
0 & 0 & 0
\end{array}\right] \mapsto\left(1+x+x^{2}\right) e} \\
& {\left[\begin{array}{lll}
0 & 1 & 0 \\
0 & 0 & 0 \\
0 & 0 & 0
\end{array}\right] \mapsto\left(1+x+x^{2}\right) y e} \\
& {\left[\begin{array}{lll}
0 & 0 & 1 \\
0 & 0 & 0 \\
0 & 0 & 0
\end{array}\right] \mapsto\left(1+x+x^{2}\right) y^{2} e} \\
& {\left[\begin{array}{lll}
0 & 0 & 0 \\
1 & 0 & 0 \\
0 & 0 & 0
\end{array}\right] \mapsto y^{2}\left(1+x+x^{2}\right) e} \\
& {\left[\begin{array}{lll}
0 & 0 & 0 \\
0 & 1 & 0 \\
0 & 0 & 0
\end{array}\right] \mapsto y^{2}\left(1+x+x^{2}\right) y e} \\
& {\left[\begin{array}{lll}
0 & 0 & 0 \\
0 & 0 & 1 \\
0 & 0 & 0
\end{array}\right] \mapsto y^{2}\left(1+x+x^{2}\right) y^{2} e} \\
& {\left[\begin{array}{lll}
0 & 0 & 0 \\
0 & 0 & 0 \\
1 & 0 & 0
\end{array}\right] \mapsto y\left(1+x+x^{2}\right) e} \\
& {\left[\begin{array}{lll}
0 & 0 & 0 \\
0 & 0 & 0 \\
0 & 1 & 0
\end{array}\right] \mapsto y\left(1+x+x^{2}\right) y e} \\
& {\left[\begin{array}{lll}
0 & 0 & 0 \\
0 & 0 & 0 \\
0 & 0 & 1
\end{array}\right] \mapsto y\left(1+x+x^{2}\right) y^{2} e} \\
& {\left[\begin{array}{lll}
\alpha & 0 & 0 \\
0 & 0 & 0 \\
0 & 0 & 0
\end{array}\right] \mapsto\left(1+x+x^{2}\right) e s} \\
& {\left[\begin{array}{lll}
0 & \alpha & 0 \\
0 & 0 & 0 \\
0 & 0 & 0
\end{array}\right] \mapsto\left(1+x+x^{2}\right) \text { yes }} \\
& {\left[\begin{array}{lll}
0 & 0 & \alpha \\
0 & 0 & 0 \\
0 & 0 & 0
\end{array}\right] \mapsto\left(1+x+x^{2}\right) y^{2} \text { es }} \\
& {\left[\begin{array}{lll}
0 & 0 & 0 \\
\alpha & 0 & 0 \\
0 & 0 & 0
\end{array}\right] \mapsto y^{2}\left(1+x+x^{2}\right) e s} \\
& {\left[\begin{array}{lll}
0 & 0 & 0 \\
0 & \alpha & 0 \\
0 & 0 & 0
\end{array}\right] \mapsto y^{2}\left(1+x+x^{2}\right) y e s} \\
& {\left[\begin{array}{lll}
0 & 0 & 0 \\
0 & 0 & \alpha \\
0 & 0 & 0
\end{array}\right] \mapsto y^{2}\left(1+x+x^{2}\right) y^{2} e s} \\
& {\left[\begin{array}{lll}
0 & 0 & 0 \\
0 & 0 & 0 \\
\alpha & 0 & 0
\end{array}\right] \mapsto y\left(1+x+x^{2}\right) e s} \\
& {\left[\begin{array}{lll}
0 & 0 & 0 \\
0 & 0 & 0 \\
0 & \alpha & 0
\end{array}\right] \mapsto y\left(1+x+x^{2}\right) \text { yes }} \\
& {\left[\begin{array}{lll}
0 & 0 & 0 \\
0 & 0 & 0 \\
0 & 0 & \alpha
\end{array}\right] \mapsto y\left(1+x+x^{2}\right) y^{2} \text { es }}
\end{aligned}
$$

Vamos determinar o ideal à esquerda em $M_{3}(\mathbb{F}(\alpha))$, gerado pelo seguinte elemento idempotente

$$
F=\left[\begin{array}{lll}
1 & 1 & 0 \\
0 & 0 & 0 \\
0 & 0 & 0
\end{array}\right]
$$

isto é,

$$
\left[\begin{array}{ccc}
a_{1}+\beta_{1} \alpha & b_{1}+\gamma_{1} \alpha & c_{1}+\delta_{1} \alpha \\
a_{2}+\beta_{2} \alpha & b_{2}+\gamma_{2} \alpha & c_{2}+\delta_{2} \alpha \\
a_{3}+\beta_{3} \alpha & b_{3}+\gamma_{3} \alpha & c_{3}+\delta_{3} \alpha
\end{array}\right] \cdot\left[\begin{array}{ccc}
1 & 1 & 0 \\
0 & 0 & 0 \\
0 & 0 & 0
\end{array}\right]=\left[\begin{array}{ccc}
a_{1}+\beta_{1} \alpha & a_{1}+\beta_{1} \alpha & 0 \\
a_{2}+\beta_{2} \alpha & a_{2}+\beta_{2} \alpha & 0 \\
a_{3}+\beta_{3} \alpha & a_{3}+\beta_{3} \alpha & 0
\end{array}\right]
$$

Este é um código com $2^{6}=64$ palavras de comprimento 27 e dimensão 6 sobre $\mathbb{F}_{2}$, e sua base é: 


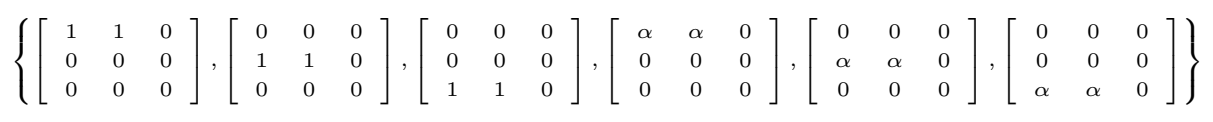

Seus respectivos elementos na álgebra $\mathbb{F} G e$ são:

$$
\begin{aligned}
& {\left[\begin{array}{lll}
1 & 1 & 0 \\
0 & 0 & 0 \\
0 & 0 & 0
\end{array}\right] \mapsto\left(1+x+x^{2}\right)(1+y) e=E} \\
& {\left[\begin{array}{lll}
\alpha & \alpha & 0 \\
0 & 0 & 0 \\
0 & 0 & 0
\end{array}\right] \mapsto E s}
\end{aligned}
$$$$
\left[\begin{array}{lll}
0 & 0 & 0 \\
1 & 1 & 0 \\
0 & 0 & 0
\end{array}\right] \mapsto y^{2} E
$$$$
\left[\begin{array}{lll}
0 & 0 & 0 \\
0 & 0 & 0 \\
1 & 1 & 0
\end{array}\right] \mapsto y E
$$$$
\left[\begin{array}{lll}
0 & 0 & 0 \\
\alpha & \alpha & 0 \\
0 & 0 & 0
\end{array}\right] \mapsto y^{2} E s
$$$$
\left[\begin{array}{lll}
0 & 0 & 0 \\
0 & 0 & 0 \\
\alpha & \alpha & 0
\end{array}\right] \mapsto y E s
$$

Logo os elementos:

$$
\left\{E, y E, y^{2} E, E s, y E s, y^{2} E s\right\},
$$

formam uma base de $\mathbb{F} G E$, onde $E$ é o idempotente lateral.

Com o uso do software livre SageMath, conforme programado, podemos computar que este código tem 36 palavras de peso 12 e 27 palavras de peso 16. Trata-se de um código de dois pesos, cujo comprimento é 27 , a dimensão é 6 e o peso é 12, igual ao melhor peso dado em codetable.de [10, Linear Codes], para este comprimento e dimensão, um código ótimo.

Exemplo 5.3. Dado $G$ um grupo do tipo $\mathscr{D}_{2}$, com $p=3$ e $m_{1}=1$ temos:

$$
G=\left\langle x, y, t_{1} ; x^{3}=y^{3}=t_{1}, t_{1}^{3}=1, s=[x, y]=t_{1}\right\rangle .
$$

Vamos explicitar as componentes simples de $\mathbb{F}_{2} G$ e um código lateral com sua respectiva distribuição de peso.

Observemos que $|G|=27$ e $G^{\prime}=\left\{1, s, s^{2}\right\}$. Vamos escrever $\mathbb{F}$ nos referindo a $\mathbb{F}_{2}$.

O conjunto das 2-classes das classes de conjugação de $G$ é:

$$
\begin{aligned}
\{ & \{1\},\left\{\{s\},\left\{s^{2}\right\}\right\}, \\
& \left\{\left\{x, x s, x s^{2}\right\},\left\{x^{2}, x^{2} s, x^{2} s^{2}\right\}\right\}, \\
& \left\{\left\{y, y s, y s^{2}\right\},\left\{y^{2}, y^{2} s, y^{2} s^{2}\right\}\right\}, \\
& \left\{\left\{x y, x y s, x y s^{2}\right\},\left\{x^{2} y^{2}, x^{2} y^{2} s, x^{2} y^{2} s^{2}\right\}\right\}, \\
& \left.\left\{\left\{x^{2} y, x^{2} y s, x^{2} y s^{2}\right\},\left\{x y^{2}, x y^{2} s, x y^{2} s^{2}\right\}\right\} \quad\right\} .
\end{aligned}
$$

Com isso, vê-se que em $\mathbb{F} G$ têm 6 componentes simples.

Agora analisemos a parte comutativa da álgebra $\mathbb{F} G$.

$$
\frac{G}{G^{\prime}}=\left\{\overline{1}, \bar{x}, \overline{x^{2}}, \bar{y}, \overline{y^{2}}, \overline{x y}, \overline{x^{2} y}, \overline{x y^{2}}, \overline{x^{2} y^{2}}\right\} .
$$

O conjunto das 2-classes é:

$$
\begin{aligned}
\{ & \{\overline{1}\},\left\{\{\bar{x}\},\left\{\overline{x^{2}}\right\}\right\},\left\{\{\bar{y}\},\left\{\overline{y^{2}}\right\}\right\} \\
& \left.\left\{\{\overline{x y}\},\left\{\overline{x^{2} y^{2}}\right\}\right\},\left\{\left\{\overline{x^{2} y}\right\},\left\{\overline{x y^{2}}\right\}\right\} \quad\right\} .
\end{aligned}
$$

Temos 5 2-classes em $G / G^{\prime}$.

Para $\xi$ uma raiz 2-ésima primitiva da unidade, temos: 


$$
\mathbb{F}\left(\frac{G}{G^{\prime}}\right) \cong \mathbb{F} \oplus \mathbb{F}(\xi) \oplus \mathbb{F}(\xi) \oplus \mathbb{F}(\xi) \oplus \mathbb{F}(\xi)
$$

de dimensão 9 sobre $\mathbb{F}$.

Como temos uma componente simples na parte não comutativa, segue que

$$
\mathbb{F} G \cong \mathbb{F} \oplus \mathbb{F}(\xi) \oplus \mathbb{F}(\xi) \oplus \mathbb{F}(\xi) \oplus \mathbb{F}(\xi) \oplus M_{3}\left(\mathbb{F}_{4}\right)
$$

Sabemos que os códigos centrais de $\mathbb{F} G$ são combinatoriamente equivalentes a abelianos, e temos interesse em trabalhar com códigos minimais laterais da parte não comutativa de $\mathbb{F} G$. $O$ peso registrado na codetable.de [10, Linear Codes] é o peso ótimo para este comprimento e dimensão. Para isso, é suficiente trabalhar com códigos minimais.

O idempotente gerador da parte não comutativa de $\mathbb{F} G$ é:

$$
e=1-e_{G^{\prime}}=1-\frac{1+s+s^{2}}{3}=s+s^{2} .
$$

Então, $\mathbb{F} G e \cong M_{3}\left(\mathbb{F}_{4}\right)$ tem dimensão 18 sobre $\mathbb{F}$;

Semelhantemente ao mostrado no exemplo anterior, uma base da álgebra $\mathbb{F} G e$ é:

$$
\begin{gathered}
\mathscr{B}=\left\{e, x e, x^{2} e, y e, y^{2} e, x y e, x^{2} y e, x y^{2} e, x^{2} y^{2} e,\right. \\
\left.e s, x e s, x^{2} e s, y e s, y^{2} e s, x y e s, x^{2} y e s, x y^{2} e s, x^{2} y^{2} e s\right\} .
\end{gathered}
$$

No que segue vamos explicitar um código desta álgebra $\mathbb{F} G e \cong M_{3}(\mathbb{F}(\alpha))$, onde $\alpha$ é uma raiz cúbica primitiva da unidade que verifica $\alpha^{2}=1+\alpha$.

Começaremos determinando a imagem de cada elemento da álgebra $\mathbb{F} G e$ com o seu correspondente elemento no anel das matrizes $M_{3}(\mathbb{F}(\alpha))$ :

$$
\begin{aligned}
& e \mapsto\left[\begin{array}{lll}
1 & 0 & 0 \\
0 & 1 & 0 \\
0 & 0 & 1
\end{array}\right] \quad x e \mapsto\left[\begin{array}{lll}
0 & 0 & \alpha \\
1 & 0 & 0 \\
0 & 1 & 0
\end{array}\right] \\
& x y e \mapsto\left[\begin{array}{ccc}
0 & \alpha & 0 \\
0 & 0 & 1 \\
\alpha & 0 & 0
\end{array}\right] \quad x^{2} e \mapsto\left[\begin{array}{ccc}
0 & \alpha & 0 \\
0 & 0 & \alpha \\
1 & 0 & 0
\end{array}\right] \quad y^{2} e \mapsto\left[\begin{array}{ccc}
0 & 1 & 0 \\
0 & 0 & \alpha \\
\alpha & 0 & 0
\end{array}\right] \quad x s^{2} e \mapsto\left[\begin{array}{ccc}
0 & 0 & 1 \\
\alpha^{2} & 0 & 0 \\
0 & \alpha^{2} & 0
\end{array}\right] \\
& x^{2} y e \mapsto\left[\begin{array}{ccc}
\alpha^{2} & 0 & 0 \\
0 & \alpha & 0 \\
0 & 0 & 1
\end{array}\right] \quad x y^{2} e \mapsto\left[\begin{array}{ccc}
\alpha^{2} & 0 & 0 \\
0 & 1 & 0 \\
0 & 0 & \alpha
\end{array}\right] \quad x^{2} y^{2} e \mapsto\left[\begin{array}{ccc}
0 & 0 & \alpha^{2} \\
\alpha^{2} & 0 & 0 \\
0 & 1 & 0
\end{array}\right] \quad y s^{2} e \mapsto\left[\begin{array}{ccc}
0 & 0 & \alpha^{2} \\
1 & 0 & 0 \\
0 & \alpha^{2} & 0
\end{array}\right] \\
& s e \mapsto\left[\begin{array}{ccc}
\alpha & 0 & 0 \\
0 & \alpha & 0 \\
0 & 0 & \alpha
\end{array}\right] \quad x s e \mapsto\left[\begin{array}{ccc}
0 & 0 & \alpha^{2} \\
\alpha & 0 & 0 \\
0 & \alpha & 0
\end{array}\right] \quad y s e \mapsto\left[\begin{array}{ccc}
0 & 0 & \alpha \\
\alpha^{2} & 0 & 0 \\
0 & \alpha & 0
\end{array}\right] \quad x y s^{2} e \mapsto\left[\begin{array}{ccc}
0 & 1 & 0 \\
0 & 0 & \alpha^{2} \\
1 & 0 & 0
\end{array}\right] \\
& x y s e \mapsto\left[\begin{array}{ccc}
0 & \alpha^{2} & 0 \\
0 & 0 & \alpha \\
\alpha^{2} & 0 & 0
\end{array}\right] \quad x^{2} s e \mapsto\left[\begin{array}{ccc}
0 & \alpha^{2} & 0 \\
0 & 0 & \alpha^{2} \\
\alpha & 0 & 0
\end{array}\right] \quad y^{2} s e \mapsto\left[\begin{array}{ccc}
0 & \alpha & 0 \\
0 & 0 & \alpha^{2} \\
\alpha^{2} & 0 & 0
\end{array}\right] \quad x^{2} s^{2} e \mapsto\left[\begin{array}{ccc}
0 & 1 & 0 \\
0 & 0 & 1 \\
\alpha^{2} & 0 & 0
\end{array}\right]
\end{aligned}
$$

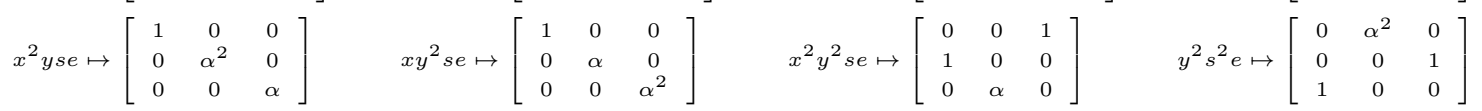

$$
\begin{aligned}
& x^{2} y s^{2} e \mapsto\left[\begin{array}{ccc}
\alpha & 0 & 0 \\
0 & 1 & 0 \\
0 & 0 & \alpha^{2}
\end{array}\right] \quad x y^{2} s^{2} e \mapsto\left[\begin{array}{ccc}
\alpha & 0 & 0 \\
0 & \alpha^{2} & 0 \\
0 & 0 & 1
\end{array}\right] \quad x^{2} y^{2} s^{2} e \mapsto\left[\begin{array}{ccc}
0 & 0 & \alpha \\
\alpha & 0 & 0 \\
0 & 1 & 0
\end{array}\right]
\end{aligned}
$$

Verifica-se que:

- o elemento $e$, identidade da álgebra $\mathbb{F} G e$ é levado na matriz identidade de ordem 3;

- as matrizes associadas a xe e ye elevada à potência 3 resultam na matriz associada ao elemento es;

- a relação $x y e s=y x e$ é verificada pelas respectivas imagens. 
Com a identificação dada anteriormente, os elementos da base canônica da álgebra das matrizes correspondem aos seguintes elementos na álgebra $\mathbb{F} G$ :

$$
\begin{aligned}
& {\left[\begin{array}{lll}
1 & 0 & 0 \\
0 & 0 & 0 \\
0 & 0 & 0
\end{array}\right] \mapsto\left(1+x y^{2} s+x^{2} y s\right) e \quad\left[\begin{array}{lll}
0 & 1 & 0 \\
0 & 0 & 0 \\
0 & 0 & 0
\end{array}\right] \mapsto\left(1+x y^{2} s+x^{2} y s\right) y^{2} e \quad\left[\begin{array}{ccc}
0 & 0 & 1 \\
0 & 0 & 0 \\
0 & 0 & 0
\end{array}\right] \mapsto\left(1+x y^{2} s+x^{2} y s\right) y e} \\
& {\left[\begin{array}{lll}
0 & 0 & 0 \\
1 & 0 & 0 \\
0 & 0 & 0
\end{array}\right] \mapsto x\left(1+x y^{2} s+x^{2} y s\right) e \quad\left[\begin{array}{lll}
0 & 0 & 0 \\
0 & 1 & 0 \\
0 & 0 & 0
\end{array}\right] \mapsto x\left(1+x y^{2} s+x^{2} y s^{2}\right) y^{2} e \quad\left[\begin{array}{ccc}
0 & 0 & 0 \\
0 & 0 & 1 \\
0 & 0 & 0
\end{array}\right] \mapsto x\left(1+x y^{2} s+x^{2} y s\right) y e} \\
& {\left[\begin{array}{lll}
0 & 0 & 0 \\
0 & 0 & 0 \\
1 & 0 & 0
\end{array}\right] \mapsto x^{2}\left(1+x y^{2} s+x^{2} y s\right) e \quad\left[\begin{array}{lll}
0 & 0 & 0 \\
0 & 0 & 0 \\
0 & 1 & 0
\end{array}\right] \mapsto x^{2}\left(1+x y^{2} s+x^{2} y s\right) y^{2} e \quad\left[\begin{array}{lll}
0 & 0 & 0 \\
0 & 0 & 0 \\
0 & 0 & 1
\end{array}\right] \mapsto x^{2}\left(1+x y^{2} s+x^{2} y s\right) y e} \\
& {\left[\begin{array}{lll}
\alpha & 0 & 0 \\
0 & 0 & 0 \\
0 & 0 & 0
\end{array}\right] \mapsto\left(1+x y^{2} s+x^{2} y s\right) s e \quad\left[\begin{array}{ccc}
\alpha^{2} & 0 & 0 \\
0 & 0 & 0 \\
0 & 0 & 0
\end{array}\right] \mapsto\left(1+x y^{2} s+x^{2} y s\right) s^{2} e}
\end{aligned}
$$

Vamos determinar o ideal à esquerda em $M_{3}(\mathbb{F}(\alpha))$ gerado pelo idempotente:

$$
F=\left[\begin{array}{lll}
1 & 1 & 1 \\
0 & 0 & 0 \\
0 & 0 & 0
\end{array}\right]
$$

isto é,

$$
\left[\begin{array}{ccc}
a_{1}+\beta_{1} \alpha & b_{1}+\gamma_{1} \alpha & c_{1}+\delta_{1} \alpha \\
a_{2}+\beta_{2} \alpha & b_{2}+\gamma_{2} \alpha & c_{2}+\delta_{2} \alpha \\
a_{3}+\beta_{3} \alpha & b_{3}+\gamma_{3} \alpha & c_{3}+\delta_{3} \alpha
\end{array}\right] \cdot\left[\begin{array}{ccc}
1 & 1 & 1 \\
0 & 0 & 0 \\
0 & 0 & 0
\end{array}\right]=\left[\begin{array}{ccc}
a_{1}+\beta_{1} \alpha & a_{1}+\beta_{1} \alpha & a_{1}+\beta_{1} \alpha \\
a_{2}+\beta_{2} \alpha & a_{2}+\beta_{2} \alpha & a_{2}+\beta_{2} \alpha \\
a_{3}+\beta_{3} \alpha & a_{3}+\beta_{3} \alpha & a_{3}+\beta_{3} \alpha
\end{array}\right] .
$$

Este é um código com 63 palavras não nulas, de comprimento 27 e de dimensão 6 sobre $\mathbb{F}_{2}$.

As matrizes da base com seus respectivos correspondentes, na álgebra $\mathbb{F} G e$ são as seguintes:

Logo os elementos:

$$
\begin{array}{llrl}
{\left[\begin{array}{lll}
1 & 1 & 1 \\
0 & 0 & 0 \\
0 & 0 & 0
\end{array}\right] \mapsto\left(1+x y^{2} s+x^{2} y s\right)\left(1+y^{2}+y\right) e=E} & {\left[\begin{array}{lll}
0 & 0 & 0 \\
1 & 1 & 1 \\
0 & 0 & 0
\end{array}\right] \mapsto x E} & {\left[\begin{array}{ccc}
0 & 0 & 0 \\
0 & 0 & 0 \\
1 & 1 & 1
\end{array}\right] \mapsto x^{2} E} \\
{\left[\begin{array}{lll}
\alpha & \alpha & \alpha \\
0 & 0 & 0 \\
0 & 0 & 0
\end{array}\right] \mapsto E s} & {\left[\begin{array}{ccc}
0 & 0 & 0 \\
\alpha & \alpha & \alpha \\
0 & 0 & 0
\end{array}\right] \mapsto x E s \quad} & {\left[\begin{array}{ccc}
0 & 0 & 0 \\
0 & 0 & 0 \\
\alpha & \alpha & \alpha
\end{array}\right] \mapsto x^{2} E s .}
\end{array}
$$

$$
\left\{E, x E, x^{2} E, E s, x E s, x^{2} E s\right\}
$$

formam uma base de $\mathbb{F} G E$, onde $E$ é o idempotente lateral.

Para computar o peso do código usando o programa SageMath, é preciso de:

- $e=s+s^{2}$, o idempotente primitivo gerador da componente simples;

- $E=\left(1+x y^{2} s+x^{2} y s\right)\left(1+y+y^{2}\right) e$, o idempotente lateral gerador do código;

- $\mathscr{B}_{E}=\left\{E, x E, x^{2} E, E s, x E s, x^{2} E s\right\}$, a base do código.

Conforme programado, temos:

$$
|G|=27
$$

1 palavra de peso 0

27 palavras de peso 12

27 palavras de peso 14

9 palavras de peso 18

Total: 64 palavras $\left(2^{6}\right)$

Trata-se de um código de comprimento 27, com dimensão 6 e com peso 12, igualado ao da codetables.de [10, Linear Codes]. 
Mostramos dois códigos com os mesmos parâmetros $(27,6,12)$, que não são equivalentes, pois não têm a mesma distribuição de pesos.

No exemplo que segue, mostramos um caso em que o gerador idempotente central, da parte não comutativa, não é primitivo e portanto, se decompõe em soma de primitivos.

Exemplo 5.4. Seja $G$ um grupo do tipo $\mathscr{D}_{1}$, com $p=7$ e $m_{1}=1$. Assim,

$$
G=\left\langle x, y, s ; x^{7}=y^{7}=s^{7}=1, s=[x, y]\right\rangle .
$$

Vamos explicitar as componentes simples da parte não comutativa de $\mathbb{F}_{2} G$ e um código lateral com a sua respectiva distribuição de peso.

Observemos que $|G|=343$ e $G^{\prime}=\left\{1, s, s^{2}, s^{3}, s^{4}, s^{5}, s^{6}\right\}$.

Pela Proposição 2.16, o conjunto das 2-classes das classes de conjugação de $\mathscr{Z}(G)$ é:

$$
\left\{\{1\},\left\{\{s\},\left\{s^{2}\right\},\left\{s^{4}\right\}\right\},\left\{\left\{s^{3}\right\},\left\{s^{6}\right\},\left\{s^{5}\right\}\right\}\right\},
$$

e como $\mathscr{Z}(G)=G^{\prime}$, o conjunto das 2-classes das classes de conjugação de $\mathscr{Z}(G) / G^{\prime}$ é: $\{\{\overline{1}\}\}$

Com isso, vê-se que em $\mathbb{F} G$ tem $3-1=2$ componentes simples na parte não comutativa.

Sabemos que

$$
\mathbb{F} G \cong \mathbb{F} G e_{G^{\prime}} \oplus \mathbb{F} G\left(1-e_{G^{\prime}}\right) .
$$

Como $\left|\frac{G}{G^{\prime}}\right|=49$ temos que $\operatorname{dim}\left(\mathbb{F} G\left(1-e_{G^{\prime}}\right)\right)=294$. Observemos que as 2-classes não triviais das classes de conjugação de $\mathscr{Z}(G)$ possuem 3 elementos e pelo Teorema 2.17, segue que

$$
\mathbb{F} G\left(1-e_{G^{\prime}}\right) \cong M_{7}\left(\mathbb{F}_{2^{3}}\right) \oplus M_{7}\left(\mathbb{F}_{2^{3}}\right) .
$$

Este é um caso que o idempotente central $\left(1-e_{G^{\prime}}\right)$ não é primitivo, pois basta olhar que temos duas 2-classes não triviais de $G^{\prime}$.

Além disso, os idempotentes primitivos da parte não comutativa são:

$$
\begin{aligned}
\left(1-e_{G^{\prime}}\right) & =s+s^{2}+s^{3}+s^{4}+s^{5}+s^{6} \\
& =\left(1+s+s^{2}+s^{4}\right)+\left(1+s^{3}+s^{5}+s^{6}\right) \\
& =e_{1}+e_{2}
\end{aligned}
$$

e

$$
e_{1} \cdot e_{2}=\left(1+s+s^{2}+s^{4}\right) \cdot\left(1+s^{3}+s^{5}+s^{6}\right)=0 .
$$

Portanto,

$$
\mathbb{F} G e_{1} \oplus \mathbb{F} G e_{2}=\mathbb{F} G\left(1-e_{G^{\prime}}\right) \cong M_{7}\left(\mathbb{F}_{2^{3}}\right) \oplus M_{7}\left(\mathbb{F}_{2^{3}}\right)
$$

No caso de 7 -grupos dos tipos $\mathscr{D}_{i}$ com $1 \leq i \leq 5$ sobre $\mathbb{F}_{2}$, o gerador idempotente central da parte não comutativa, se decompõe na soma mostrada acima.

Semelhantemente ao mostrado nos exemplos anteriores, a base da álgebra $\mathbb{F} G e_{1}$ tem $49 \times 3=147$ elementos, e uma base desta álgebra é :

$$
\mathscr{B}=\left\{x^{i} y^{j} e_{1}, x^{i} y^{j} e_{1} s, x^{i} y^{j} e_{1} s^{2} ; 0 \leq i, j<7\right\} .
$$


Vamos determinar o ideal à esquerda em $M_{7}(\mathbb{F}(\alpha))$, onde $\alpha$ é uma raiz cúbica primitiva da unidade. Tal ideal é gerado pelo idempotente $\sum_{j=1}^{6} F_{1 j}$ que é a soma das matrizes elementares $M_{7}(\mathbb{F})$.

Lembrando que: $\mathbb{F}(\alpha)=\left\{a_{0}+a_{1} \alpha+a_{2} \alpha^{2} ; a_{i} \in \mathbb{F}, 0 \leq i \leq 2\right\}$.

O ideal, à esquerda, $\left\langle\sum_{j=1}^{6} F_{1 j}\right\rangle=\left[\left(a_{0}+a_{1} \alpha+a_{2} \alpha^{2}\right) \sum_{j=1}^{6} F_{i j}\right]$, para cada $1 \leq i \leq 7$.

Segue que a base do código é dada por $\sum_{j=1}^{6} \alpha^{r} F_{i j}$, para cada $0 \leq r \leq 2$ e $1 \leq i \leq 7$. Consequentemente a base do código tem $21=3 \times 7$ elementos, ou seja, um código de $2^{21}$ palavras.

E os seus correspondentes elementos na álgebra $\mathbb{F} G e_{1}$, são:

Para $\sum_{j=1}^{6} F_{1 j} \mapsto\left(\sum_{r=0}^{6} x^{r}\right)\left(\sum_{r=0}^{6} y^{r}\right) e_{1}=E$

$\sum_{j=1}^{6} \alpha^{r} F_{i j} \mapsto y^{j-1} E s^{r}$, para cada $0 \leq r \leq 2$ e $1 \leq i \leq 7$.

Logo os elementos:

$$
\left\{y^{i} E, y^{i} E s, y^{i} E s^{2} ; 0 \leq i \leq 6\right\},
$$

formam uma base de $\mathbb{F} G E$, onde $E$ é o idempotente lateral.

Para computar o peso do código usando o programa SageMath, é preciso de:

- $e_{1}=1+s+s^{2}+s^{4}$, o idempotente primitivo gerador da componente simples;

- $E=\left(\sum_{r=0}^{6} x^{r}\right)\left(\sum_{r=0}^{6} y^{r}\right) e_{1}$, o idempotente lateral gerador do código;

- $\mathscr{B}_{E}=\left\{y^{i} E, y^{i} E s, y^{i} E s^{2} ; 0 \leq i \leq 6\right\}$, a base do código.

Conforme programado, temos:

$$
|G|=343
$$

1 palavra de peso 0

49 palavras de peso $\mathbf{2 8}$

1029 palavras de peso 56

12005 palavras de peso 84

84035 palavras de peso 112

352947 palavras de peso 140

823543 palavras de peso 168

823543 palavras de peso 196

Total: 2097152 palavras $\left(2^{21}\right)$

Trata-se de um código de comprimento 343, a dimensão é 21 e o peso é 28. Neste caso, pelo comprimento não temos comparação em codetables.de [10, Linear Codes].

De maneira análoga ao exposto acima, apresentamos a distribuição de peso de alguns códigos desta álgebra explicitando os idempotentes laterais geradores dos mesmos, naturalmente com o uso do software SageMath.

$$
\begin{gathered}
\text { Idempotente: } E_{1}=\left(1+x^{2}+x^{3}+x^{4}+x^{5}+x^{6}\right) e \\
1 \text { palavra de peso } 0 \\
49 \text { palavras de peso } 28 \\
1029 \text { palavras de peso } 56 \\
12005 \text { palavras de peso } 84
\end{gathered}
$$


84035 palavras de peso 112 352947 palavras de peso 140 823543 palavras de peso 168 823543 palavras de peso 196 Total: 2097152 palavras $\left(2^{21}\right)$

Idempotente: $E_{1}(1+y)$

1 palavra de peso 0

49 palavras de peso 56

343 palavras de peso 80

2401 palavras de peso 104

686 palavras de peso 112

16807 palavras de peso 128

7203 palavras de peso 136

117649 palavras de peso 152

50421 palavras de peso 160

825944 palavras de peso 168

823543 palavras de peso 176

235298 palavras de peso 184

16807 palavras de peso 192

Total: 2097152 palavras $\left(2^{21}\right)$

Idempotente: $E_{1}\left(1+y+y^{2}\right)$

1 palavra de peso 0

49 palavras de peso 84

343 palavras de peso 104

1029 palavras de peso 124

1372 palavras de peso 132

343 palavras de peso 136

3234 palavras de peso 140

3087 palavras de peso 144

15435 palavras de peso 148

36015 palavras de peso 152

94325 palavras de peso 156

153664 palavras de peso 160

261366 palavras de peso 164

296695 palavras de peso 168

393421 palavras de peso 172

311444 palavras de peso 176

294637 palavras de peso 180

107016 palavras de peso 184

105987 palavras de peso 188 
17689 palavras de peso 196

Total: 2097152 palavras $\left(2^{21}\right)$

Idempotente: $E_{1}\left(1+y+y^{2}+y^{3}\right)$

1 palavra de peso 0

196 palavras de peso 112

1372 palavras de peso 128

13720 palavras de peso 144

24696 palavras de peso 148

27440 palavras de peso 152

98784 palavras de peso 156

142345 palavras de peso 160

205800 palavras de peso 164

387100 palavras de peso 168

351232 palavras de peso 172

327222 palavras de peso 176

255192 palavras de peso 180

166012 palavras de peso 184

65856 palavras de peso 188

21952 palavras de peso 192

8232 palavras de peso 196

Total: 2097152 palavras $\left(2^{21}\right)$

Idempotente: $E_{1}\left(1+y+y^{2}+y^{-3}+y^{3}\right)$

1 palavra de peso 0

686 palavras de peso 132

4165 palavras de peso 140

343 palavras de peso 144

30870 palavras de peso 148

41846 palavras de peso 152

101528 palavras de peso 156

138915 palavras de peso 160

238728 palavras de peso 164

291207 palavras de peso 168

404740 palavras de peso 172

277144 palavras de peso 176

331681 palavras de peso 180

145775 palavras de peso 184

67571 palavras de peso 188

13377 palavras de peso 192

8575 palavras de peso 196

Total: 2097152 palavras $\left(2^{21}\right)$ 
Idempotente: $E_{1}\left(1+y+y^{-2}+y^{2}+y^{-3}+y^{3}\right)$

1 palavra de peso 0

5488 palavras de peso 136

107016 palavras de peso 152

343686 palavras de peso 160

519008 palavras de peso 168

798504 palavras de peso 176

271656 palavras de peso 184

51793 palavras de peso 192

Total: 2097152 palavras $\left(2^{21}\right)$

Trata-se de códigos de comprimento 343, a dimensão é 21 e o peso é 136 para o idempotente $E_{1}\left(1+y+y^{-2}+y^{2}+y^{-3}+y^{3}\right)$. Pelo comprimento não temos comparação em codetables.de [10, Linear Codes], mas chamamos a atenção no valor do peso com relação ao idempotente. 


\section{Capítulo 6}

\section{Roteiro para o software SageMath}

Este Capítulo é dedicado a expor os comandos usados no software SageMath, conforme explicitado na Introdução deste trabalho.

\subsection{Comandos para o SageMath}

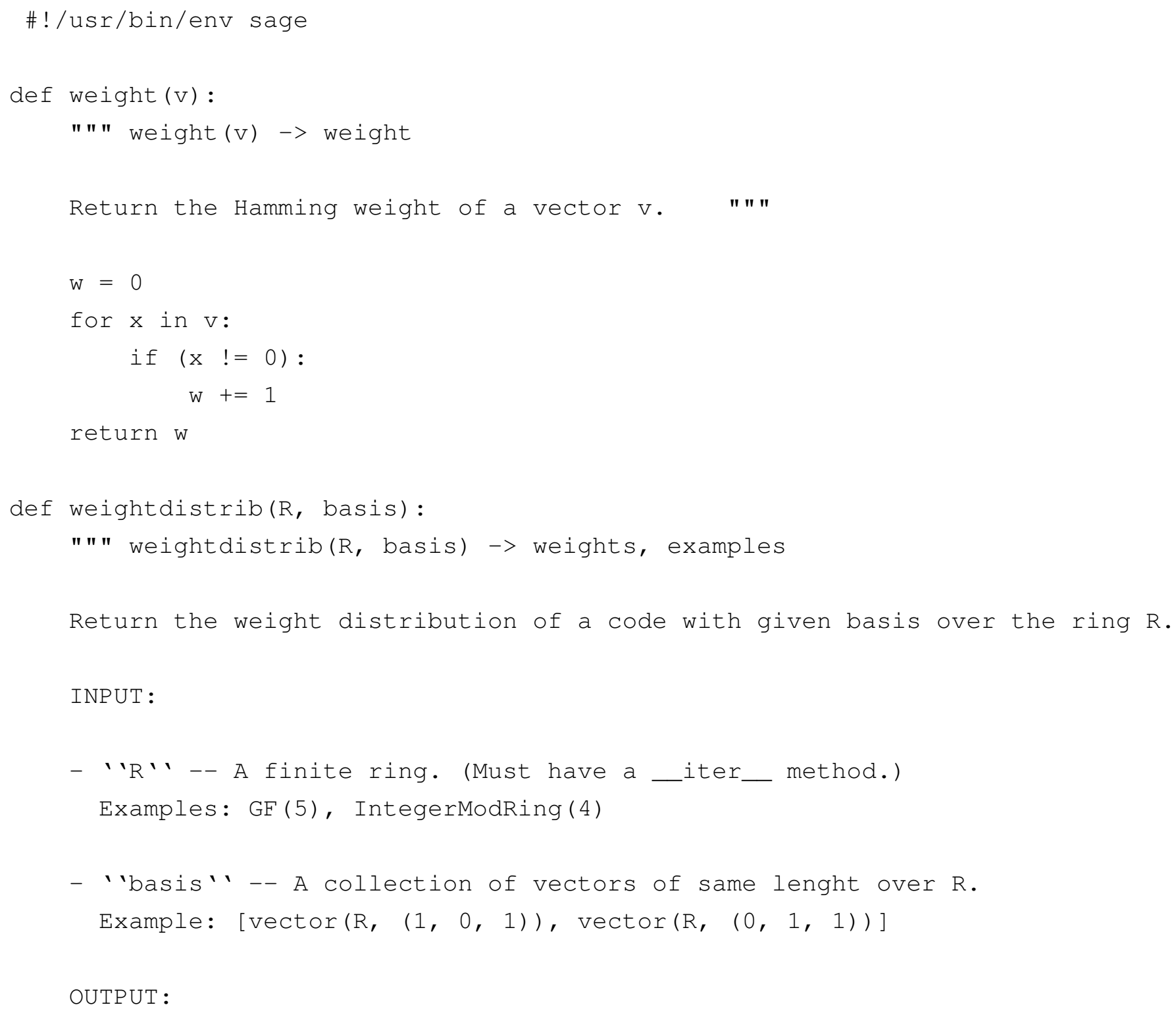


- A dictionary whose keys are the weights found and whose values are the number of words with corresponding weight.

- A dictionary whose keys are the weights found and whose values are pairs of vectors, the first being a codeword with correspondig weight and the second being the basis elements coefficients that produce this codeword.

EXAMP LE :

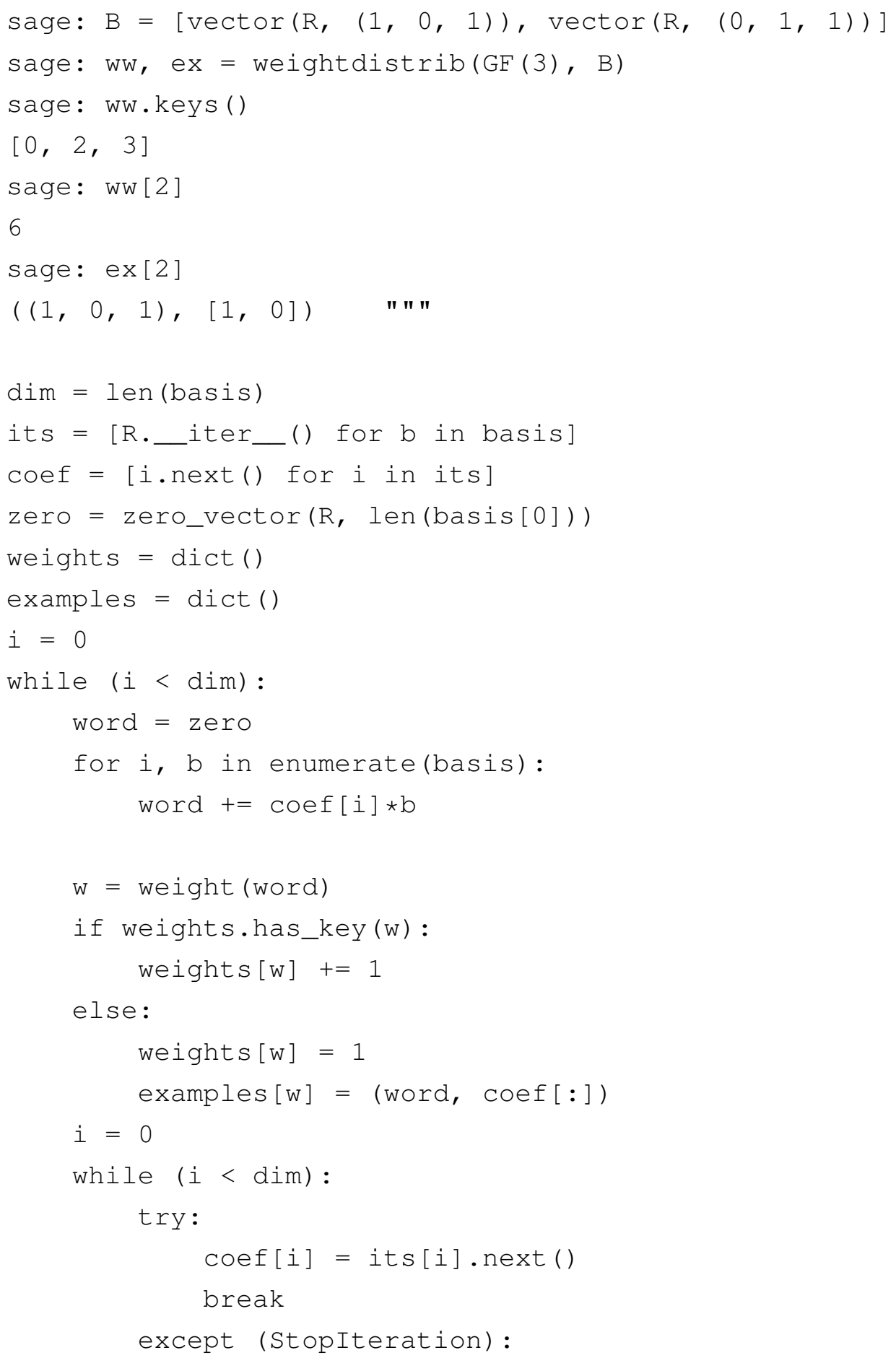




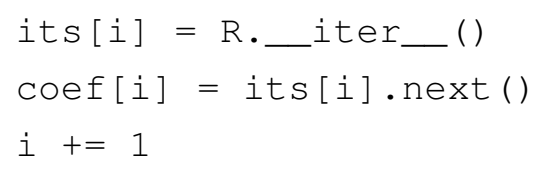

return weights, examples

\subsection{Algoritmo SageMath}

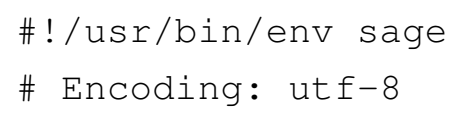

\# Subespaço de $K^{\wedge} n$ (computação mais eficiente que em KG) 


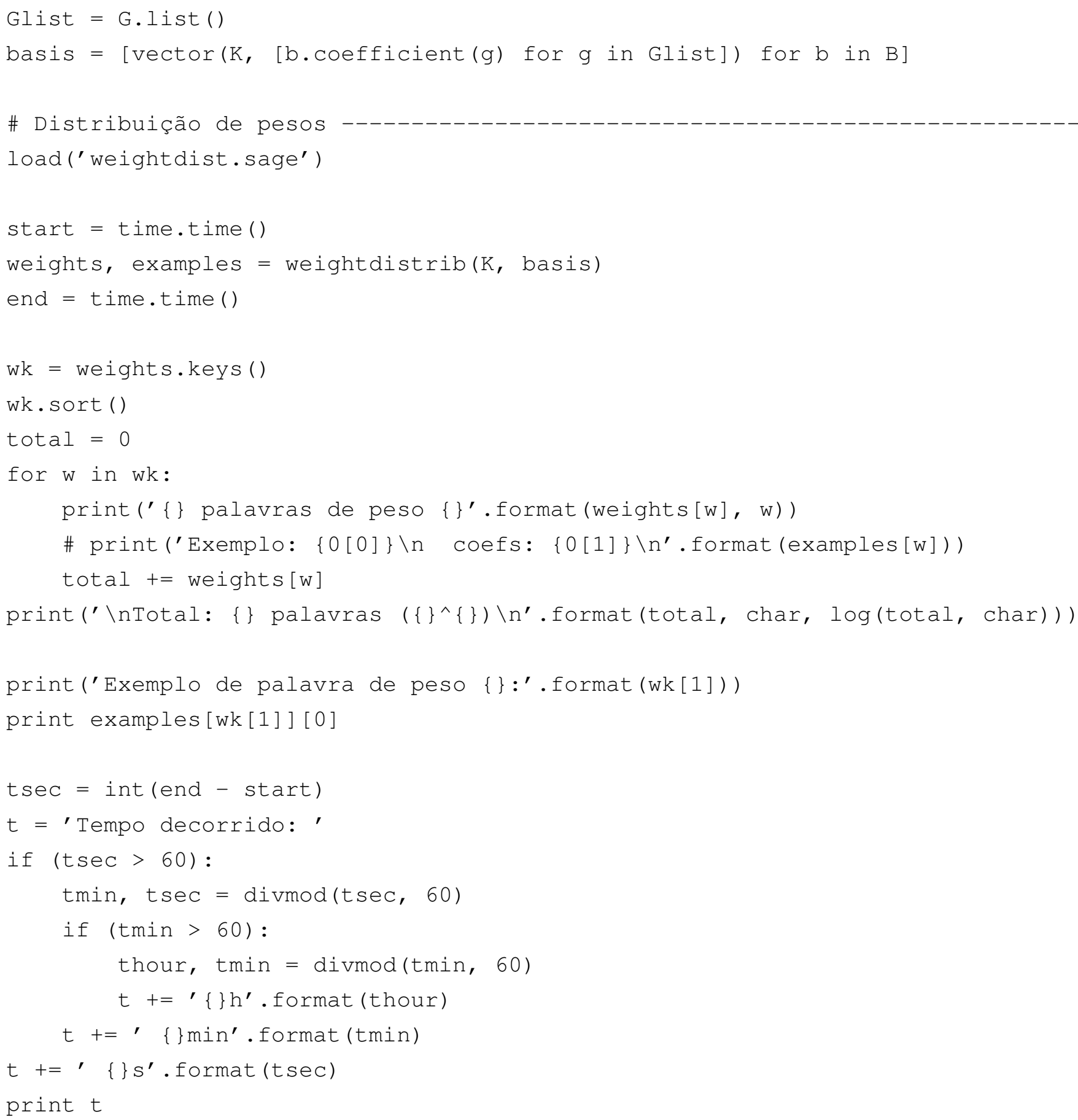




\section{Referências Bibliográficas}

[1] S. Assuena, Códigos metacíclicos, Tese de doutorado da Universidade de São Paulo, São Paulo, 2013.

[2] S. Assuena and C. Polcino Milies, Group algebras of metacyclic groups over finite fields, São Paulo Journal of Mathematical Sciences (2016), 1-7.

[3] M. G. Cornelissen and C. Polcino Milies, Finitely Generated Groups G such that $G / \mathscr{Z}(G) \cong$ $C_{p} \times C_{p}$, Comm. Algebra, v. 42 (2014), 378-388.

[4] R. A. Ferraz, Simple components of the center of $\mathbb{F} G / J(\mathbb{F} G)$, Comm. Algebra, v. 36, n. 9 (2008), 3191-3199.

[5] R. A. Ferraz, E. G. Goodaire and C. Polcino Milies, Some classes of semisimple group (and loop) algebras over finite fields, Journal of Algebra v. 324, n. 12 (2010), 3457-3469.

[6] R. A. Ferraz and C. Polcino Milies, Idempotents in Group Algebras and Minimal Abelian Codes, Finite Filds and Appl., v. 13 (2007), 382-393.

[7] M. J. Golay. Notes on digital coding. Proceedings of the Institute of Radio Engineers, 37(6) (1949), 657-657.

[8] E. G. Goodaire, Alternative loop rings, Publ. Math Debrecen 30 (1983), p. 31-38.

[9] E. G. Goodaire, E. Jespers, and C. Polcino Milies, Alternative Loop Rings, North-Holland Math. Stud., v. 84, Elsevier, Amsterdam, 1996.

[10] M. Grassl, tabela de códigos disponível em http://www.codetables.de, 2007. Acessado em $\mathbf{1 8 / 1 2 / 2 0 1 7 , ~ p . ~ 1 0 - 0 7 , ~} 2008$.

[11] R. W. Hamming, Error detecting and error correcting codes. Bell Labs Technical Journal v. 29.2 (1950), 147-160.

[12] M. I. Isaacs, Character Theory of Finite Groups, Academic Press, New York, 1976.

[13] G. James and M. W. Liebeck. Representations and characters of groups. Cambridge University Press, 2001.

[14] E. Jespers, G. Leal and C. Polcino Milies, Classifying indecomposable RA loops. Journal of Algebra, v. 176, n. 2 (1995), 569-584.

[15] G. Leal and C. Polcino Milies, Isomorphic group (and loop) algebras, J. Algebra, v. 155, n. 1 (1993), 195-210. 
[16] J. H. van Lint, and R. M. Wilson, A course in combinatorics. Cambridge University Press, 2001.

[17] C. Polcino Milies and S. K. Sehgal, An Introduction to Group Ring, Kluwer Academic Publishers, Dordrecht 2002.

[18] J. J. Rotman, An Introduction to the Theory Of Groups, fourth ed., Springer-Verlag, New York, 1995.

[19] R. E. Sabin and S. J. Lomonaco, Metacyclic Error-Correcting Codes, Applicable Algebra in Engineering, Comunication and Computing v. 6 (1995), 191-210.

[20] C. E. Shannon, Communication theory of secrecy systems. Bell Labs Technical Journal v. 28.4 (1949): 656-715.

[21] S. H. Weintraub, Representation Theory of Finite Groups: Algebra and Arithmetic, Graduate Studies in Mathematics, v. 59. American Mathematical Society, Providence, Rhode Island, 2003. 